\title{
THERMAL ANALYSIS OF MICROHOTPLATES USING COMPUTER AIDED DESIGN AND SIMULATION SOFTWARE
}

\author{
By \\ Hiren Vrajlal Trada \\ B.E. University of Mumbai, 1999

\begin{abstract}
A Thesis
Submitted to the faculty of the

Graduate School of the University of Louisville in Partial fulfillment of the Requirements

for the Degree of
\end{abstract} \\ Master of Science \\ Department of Electrical and Computer Engineering \\ University of Louisville \\ Louisville, Kentucky
}

May 2004 
THERMAL ANALYSIS OF MICROHOTPLATES USING COMPUTER AIDED DESIGN AND SIMULATION SOFTWARE

By

Hiren Vrajlal Trada

B.E. University of Mumbai, 1999

A Thesis Approved on

August 14, 2003

by the following Thesis Committee:

Thesis Director 


\section{DEDICATION}

To my parents;

to 'mummy',

for all the love and unconditional support that only a mother can give.

to 'papa';

who has been and remains an inspiration to me for life.

If not for them, I wouldn't be pursuing graduate studies. 


\section{ACKNOWLEDGMENTS}

First and foremost, I would like to thank my advisor, Dr. Kevin Walsh who has supported and guided me throughout my master's work. He has shown infinite patience and understanding over the past three years.

Thanks also to my other committee members, Dr. John Naber and Dr. Ellen Brehob, for their assistance and invaluable help in this research work, for all their comments, inputs and suggestions.

I would like to express my gratitude to Michael Martin who has been involved in this project from the beginning and has been available whenever I needed him.

Lastly, I would like to thank my family in India who have always encouraged me and supported my work even though it kept me away for over three years. 


\title{
ABSTRACT \\ THERMAL ANALYSIS OF MICROHOTPLATES USING COMPUTER AIDED DESIGN AND SIMULATION SOFTWARE
}

\author{
Hiren Trada
}

August 14, 2003

This thesis is based on work done as part of a bigger project supported by Naval Research Laboratories (NRL). A MOSIS fabricated micro hotplate is simulated using the CAD software CoventorWare. There are three different sized hotplates in one die; viz. small, medium and large.

The files designed in L-EDIT, are imported into CoventorWare which is used to build a 3-D model and perform various simulations on the models. A voltage sweep is applied to the hotplate terminals for different values of convection coefficients and the resulting temperature data is gathered. This data is compared with experimental data to obtain the best possible match.

The main goal of this work is to obtain a suitable value of convection coefficients that provide a temperature solution which is as close as possible to the experimental data. Two sets of values for the convection coefficients are used, one from previous works and the other set is calculated, based on the hotplate dimensions. 
It is found that for the small and medium sizes, the error curves are close together for different values of the coefficients while for the large hotplate, the curves are apart. Hence convective heat loss decreases with size with conduction coming into prominence. 


\section{TABLE OF CONTENTS}

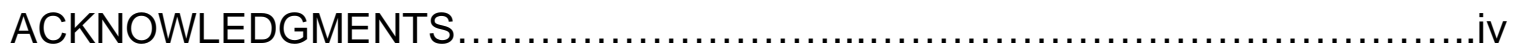

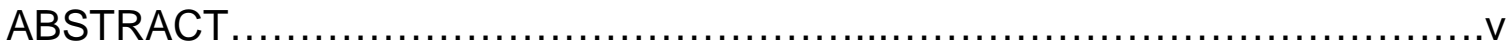

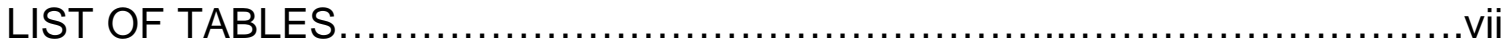

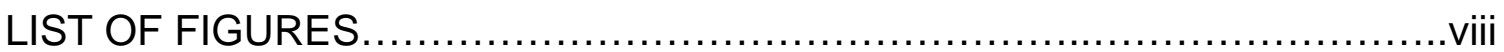

CHAPTER PAGE

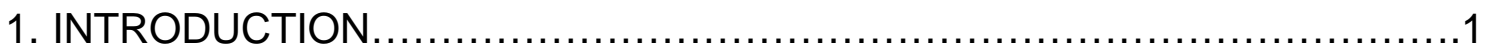

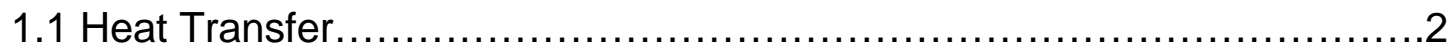

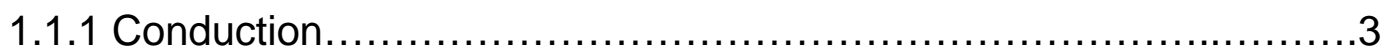

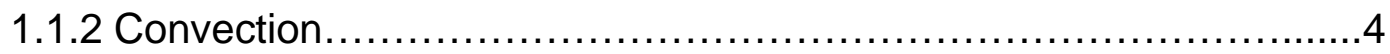

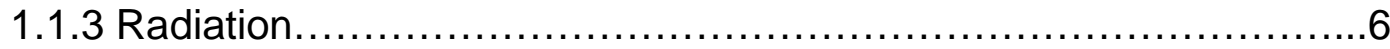

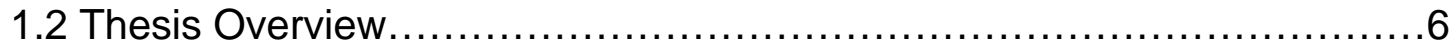

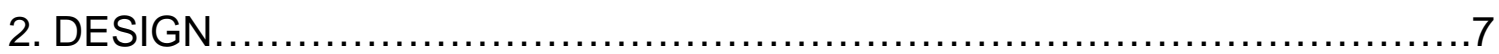

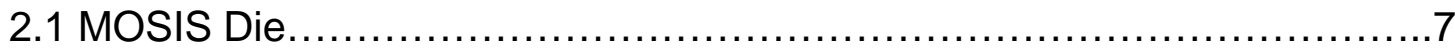

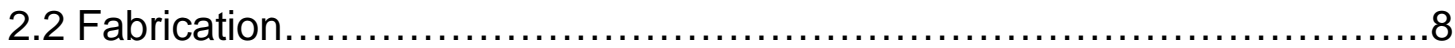

2.3 Design Masks and Dimensions.......................................... 10

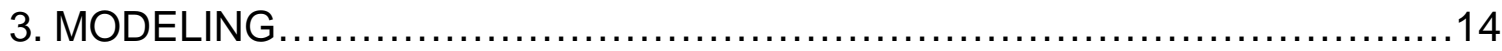

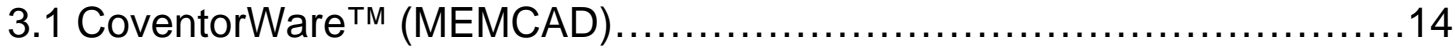

3.2 Software Reliability .............................................................

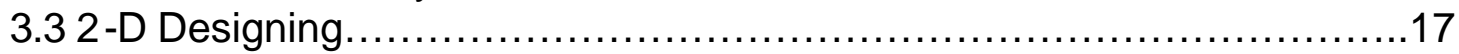

3.4 3-D Model, Meshing and Simulation................................... 17

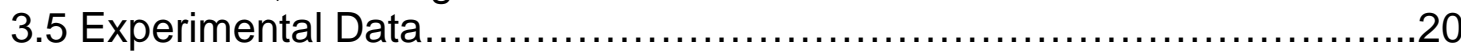

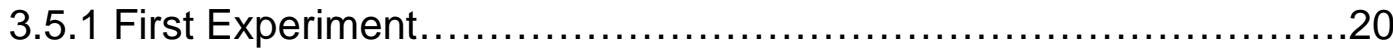

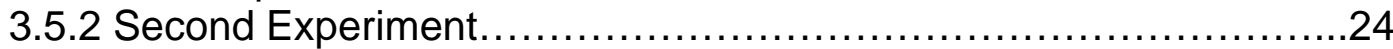

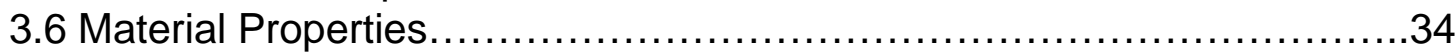

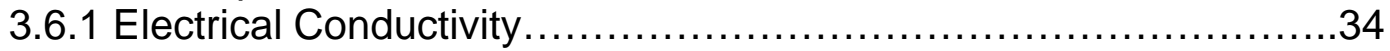

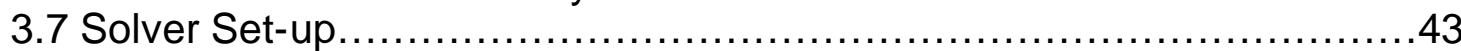

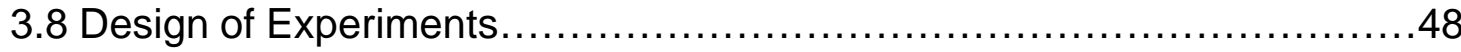

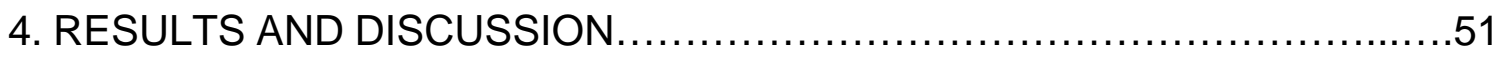

5. CONCLUSIONS AND RECOMMENDATIONS ...............................60

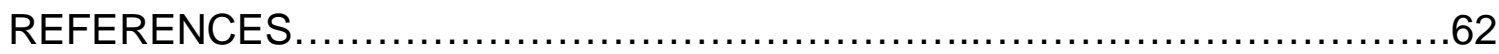

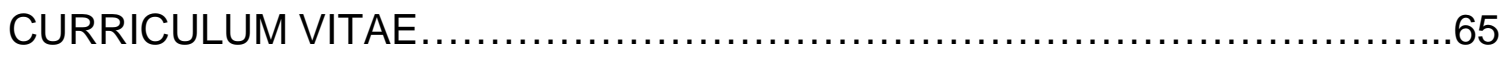




\section{LIST OF TABLES}

TABLE

PAGE

1. Dimensions of the hotplate components..................................12

2. Mesher settings for Small hotplate .....................................21

3. Mesher settings for Medium hotplate ..................................22

4. Mesher settings for Large hotplate .....................................23

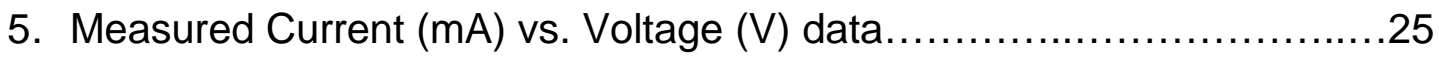

6. Calculated Resistance (O) vs. Voltage (V) data ..........................26

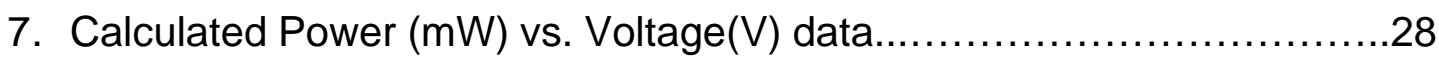

8. Measured Resistance (O) vs. Temperature $\left({ }^{\circ} \mathrm{C}\right)$ data.....................29

9. Deduced Temperature $\left({ }^{\circ} \mathrm{C}\right)$ vs. Voltage $(\mathrm{V})$ data...........................

10. Deduced Temperature $\left({ }^{\circ} \mathrm{C}\right)$ vs. Power $(\mathrm{mW})$ data .........................32

11. Electrical Conductivity of polysilicon heaters..............................40

12. Electrical conductivity values for CoventorWare ..........................42

13. Thermal Conductivity of aluminum ......................................42

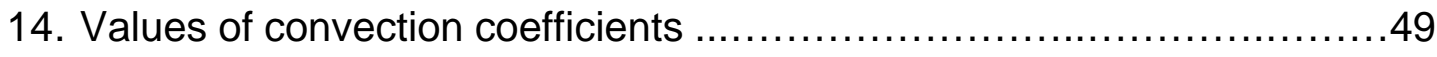

15. Calculated values of convection coefficients .............................50

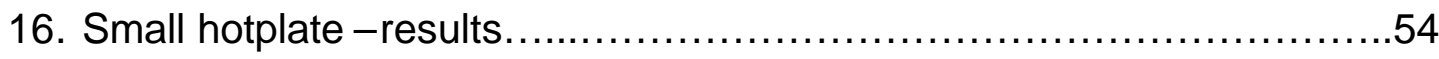

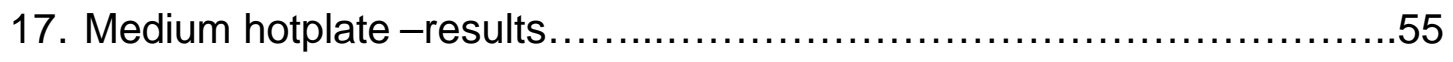

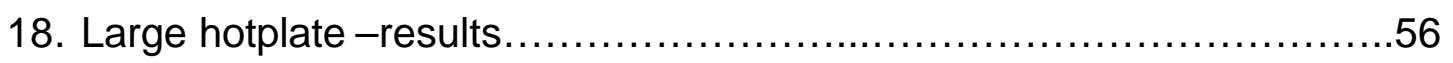




\section{LIST OF FIGURES}

FIGURE

PAGE

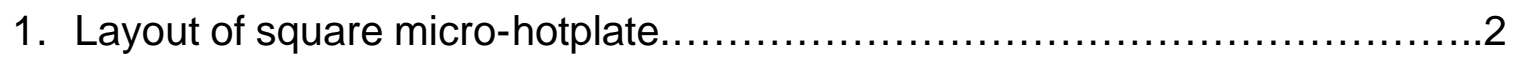

2. Conduction, Convection and Radiation modes of heat transfer .................4

3. Velocity and Temperature distributions over a heated surface .................5

4. Photograph of actual MOSIS fabricated chip looking from the top...............8

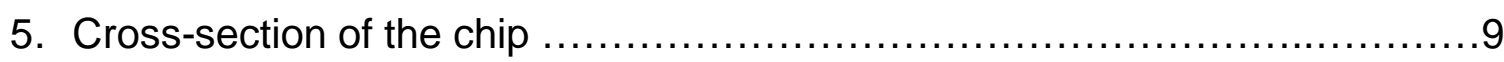

6. SEM photograph of actual released micro hotplate device ....................10

7. Mask design for the polysilicon heater......................................11

8. Mask design for the aluminum distribution plate............................11

9. Mask design for the oxide layer...........................................

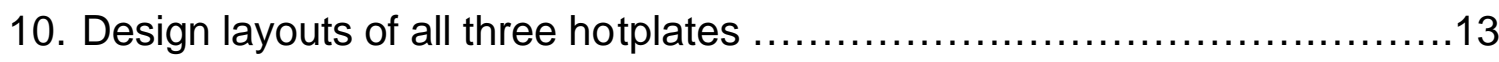

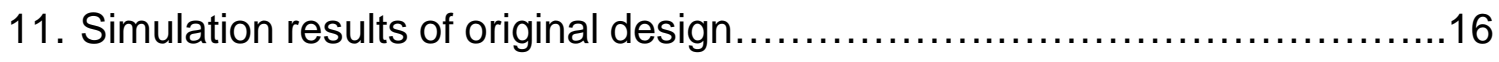

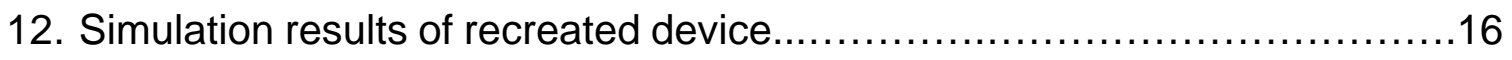

13. The CoventorWare process file for the hotplates ...........................18

14. 3-D solid models of the micro-hotplates..................................... 19

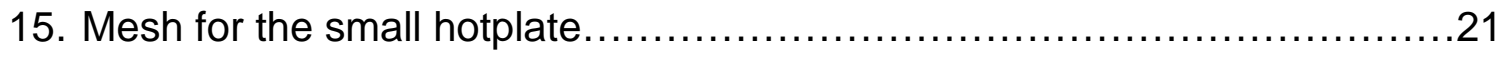

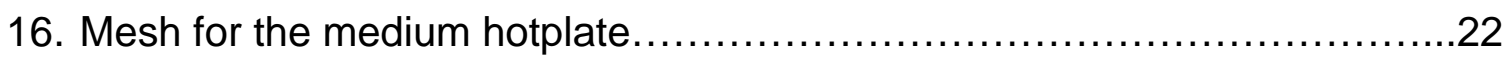

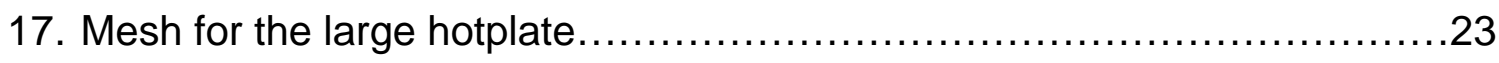

18. Measured Current (mA) vs. Voltage (V) curves...........................27 
19. Calculated Resistance (O) vs. Voltage (V) curves

20. Calculated Power (mW) vs. Voltage (V) curves.............................30

21. Measured Resistance (O) vs. Temperature $\left({ }^{\circ} \mathrm{C}\right)$ curves .....................30

22. Deduced Temperature $\left({ }^{\circ} \mathrm{C}\right)$ vs. Voltage $(\mathrm{V})$ curves ..........................33

23. Deduced Temperature $\left({ }^{\circ} \mathrm{C}\right)$ vs. Power $(\mathrm{mW})$ curves ........................33

24. Calculation of number of squares for 'small' heater.........................37

25. Calculation of number of squares for 'medium' heater........................38

26. Calculation of number of squares for 'large' heater...........................39

27. Electrical Conductivity of polysilicon......................................... 41

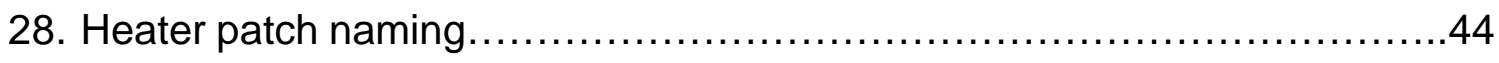

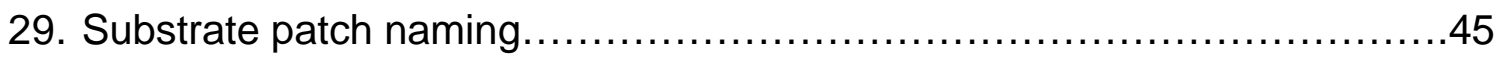

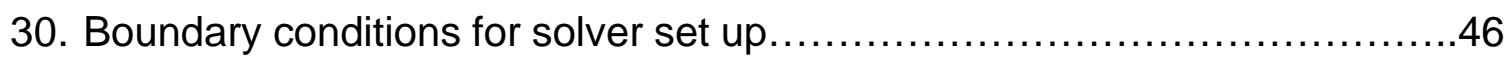

31. Setting convection boundary conditions ..................................46

32. Electro-Thermal boundary conditions for parametric solution.................47

33. Trajectory 't1' with 'delta' variation .......................................48

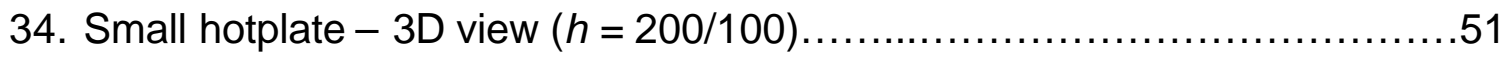

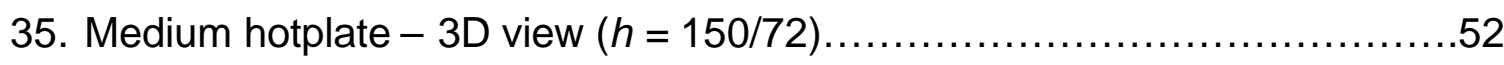

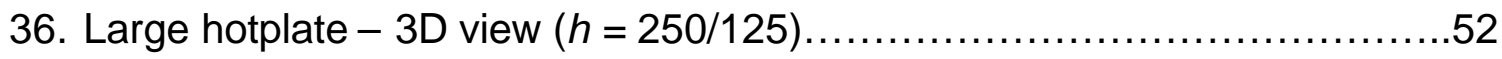

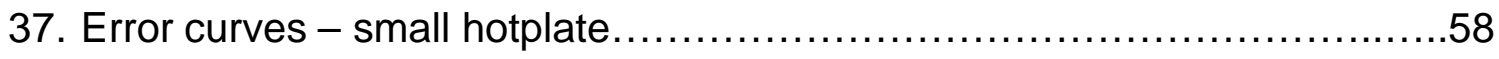

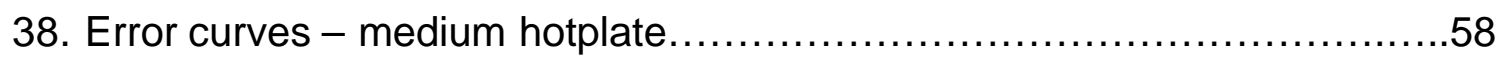

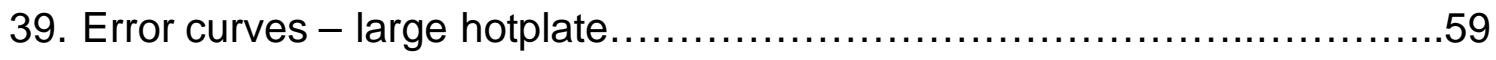




\section{CHAPTER 1 \\ INTRODUCTION}

This thesis is based on research work done as part of a project supported by Naval Research Laboratory (NRL), Washington, D.C. The project involves a MEMS device, which is used in the front end of an explosive detector. MEMS is

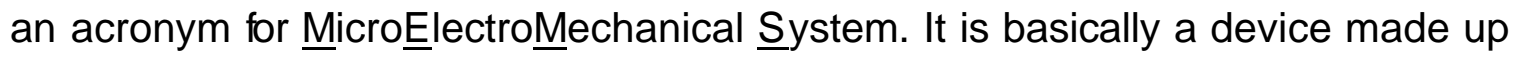
of electronic and mechanical systems at the micron scale. The explosive detector uses a pre-concentrator at the front end for trace detection of chemical particles. The MEMS device used for the pre-concentrator is a micro-hotplate.

There are two universities working on this project apart from NRL, University of Louisville (UofL) and George Washington University (GWU). UofL fabricates custom-designed hotplates while GWU designs hotplates that are fabricated through MOSIS. Since MOSIS is a commercial foundry, which fabricates hundreds of different designs on one wafer, the design and the choice of materials is limited. The MOSIS hotplates are designed by loana Voiculescu, a doctoral student at GWU.

This research uses the GWU designed file and simulates the hotplates in the MEMS software, Coventorware. Coventorware is a powerful MEMS tool with a full-featured suite of programs. The software has the ability to design a device, 
build a 3-D model and perform various simulations on the MEMS device. It has integrated solvers for different kinds of MEMS devices.

The heater is embedded in a structure, which is separated and suspended over the silicon substrate (see Figure 1). Thus the device is in contact with air at the top and bottom surfaces. Since there is a temperature difference between the surface and the fluid (air), heat transfer takes place.

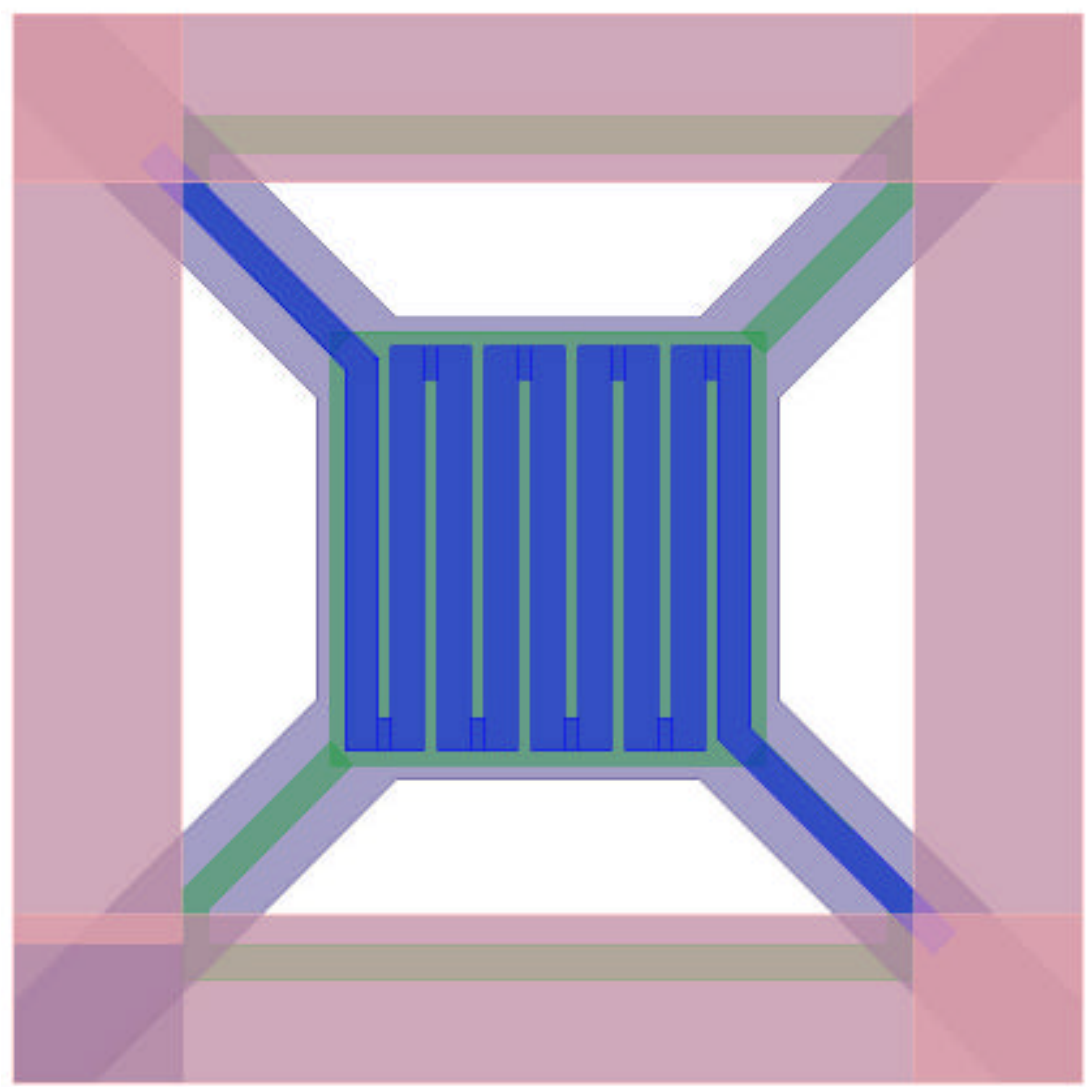

Figure 1: Layout of square micro-hotplate [6]

\subsection{Heat Transfer}

Heat or heat transfer is defined as the transfer of energy in a medium or between two or more media due to a difference in temperature [1, 2]. Figure 2 shows the different modes of heat transfer. 


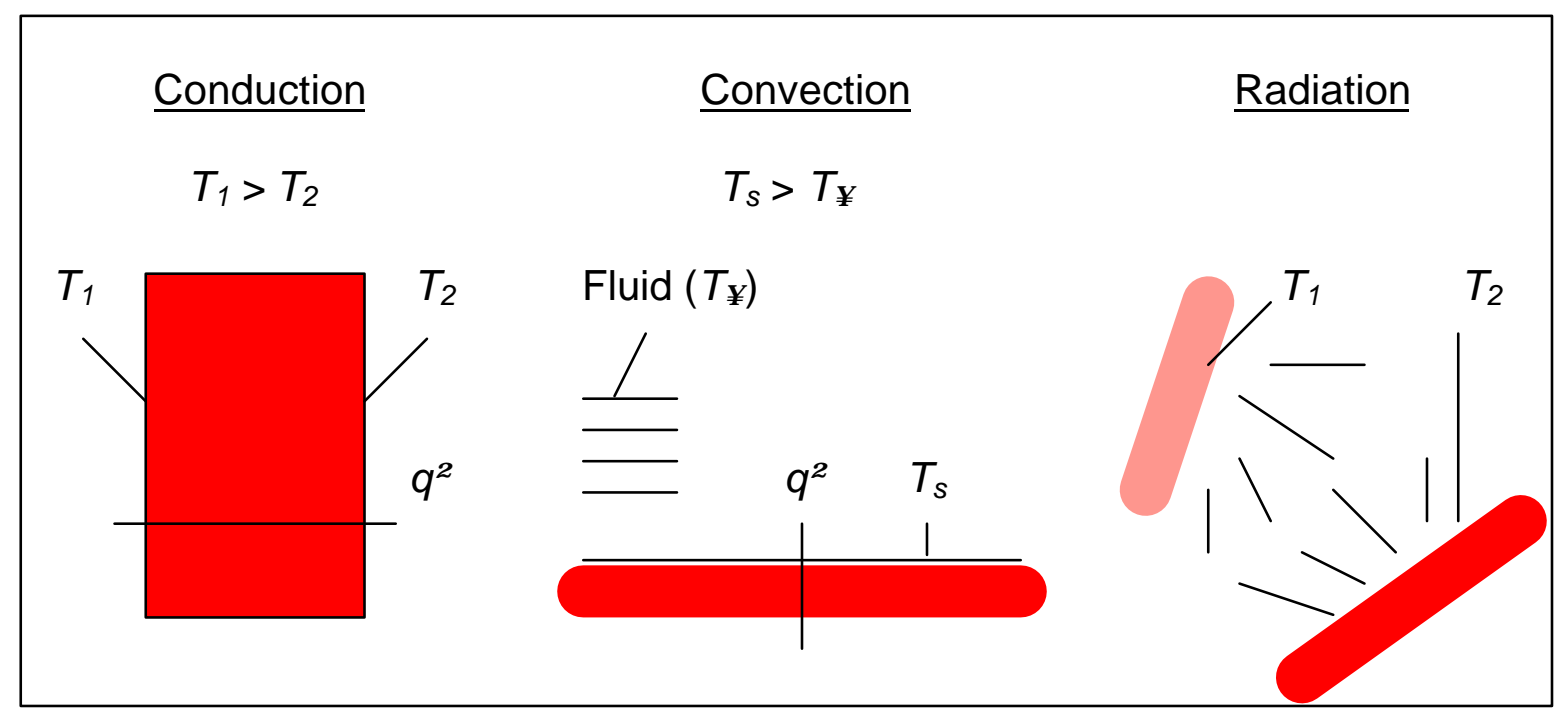

Figure 2: Conduction, Convection and Radiation modes of heat transfer [1].

When heat transfer takes place within a medium it is referred to as conduction. Convection is defined as heat transfer between a surface and a fluid (in contact), when there is bulk motion of the fluid, at different temperatures. When heat transfer occurs between two surfaces (not in contact) it is termed as Radiation. Radiation is possible due to electromagnetic waves that are emitted by all surfaces at a finite temperature.

\subsubsection{Conduction}

Conduction in a medium occurs due to random molecular motion. For example, in a gas (or liquid) medium, the molecules in the high temperature region possess more kinetic energy than molecules in the low temperature region. These high energy molecules are therefore in constant motion and collide with other molecules thereby transferring energy. In solid medium (metals) this transfer of energy takes place largely due to the motion of electrons. 
To quantify the heat transfer process and to compute the energy exchanged, we use rate equations. Consider Figure 2; for one-dimensional conduction through the wall with (its two surfaces at) temperatures $T_{1}$ and $T_{2}$, the rate equation $[4,5]$ is

$$
q^{\prime \prime}=-k(d T / d x)
$$

where: $q^{\prime \prime}=$ heat flux $\left(\mathrm{W} / \mathrm{m}^{2}\right)$

$k=$ thermal conductivity $(\mathrm{W} / \mathrm{m} . \mathrm{K})$

$(d T / d x)=$ temperature gradient

Equation 1 can be written as,

$$
q^{\prime \prime}=-k\left(T_{1}-T_{2}\right) / t
$$

where: $(d T / d x)=\left(T_{1}-T_{2}\right) / t$

$t=$ wall thickness $(\mathrm{m})$

For this research, the wall (arms) thickness is the thickness of the (6) layers which is $3.7 \mu \mathrm{m}$ (Figure 5). Conductive heat transfer is assumed as negligible since there is no significant temperature variation between the two surfaces.

\subsubsection{Convection}

Convection occurs between a surface and a fluid, which are at two different temperatures. Fluid - surface interaction (see Figure 3) results in a region known as hydrodynamic/velocity boundary layer. The velocity of this layer varies from ' 0 ' near the surface to $u_{\infty}$, an average for the fluid. Temperature at surface is $T_{s}$ and fluid temperature far from the wall is $T_{\infty}$. This region is known as thermal boundary layer. Convection heat transfer will occur if $T_{s}$ and $T_{\infty}$ are different. 


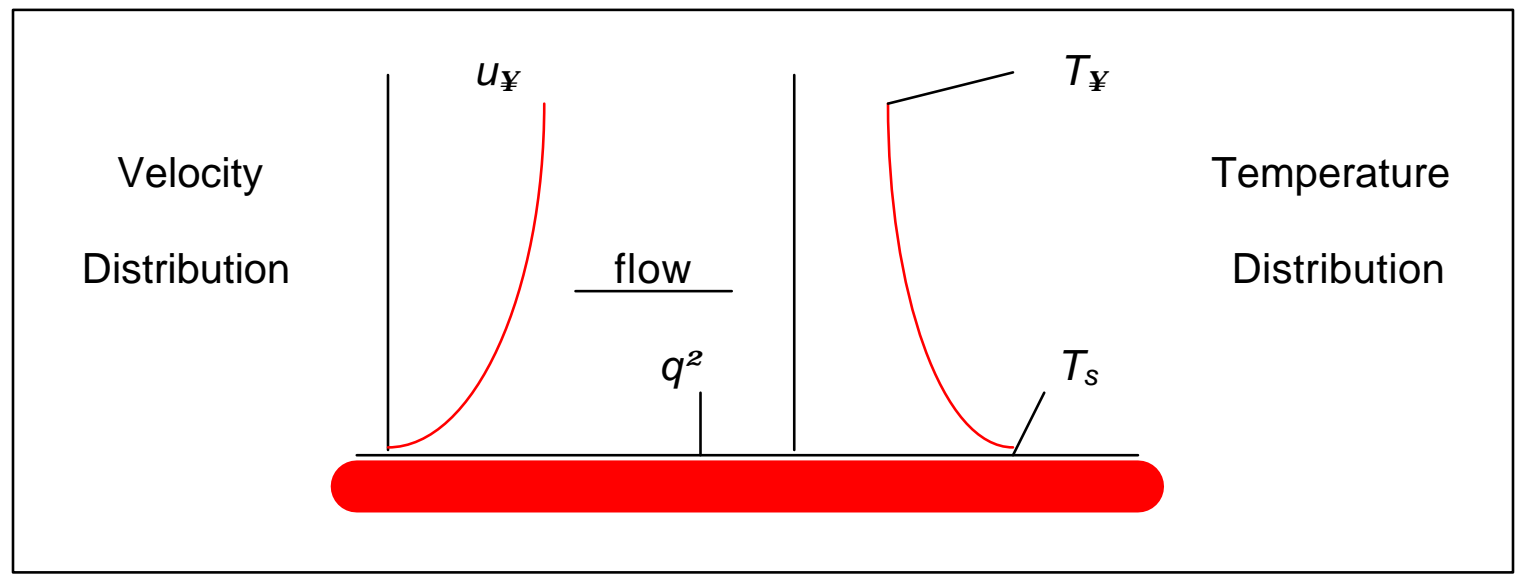

Figure 3: Velocity and Temperature distributions over a heated surface $[6,7]$

The rate equation is defined as

$$
q^{\prime \prime}=h\left(T_{s}-T_{\infty}\right)
$$

where: $q^{\prime \prime}=$ convective heat flux $\left(\mathrm{W} / \mathrm{m}^{2}\right)$

$h=$ Convection heat transfer coefficient $\left(\mathrm{W} / \mathrm{m}^{2} \cdot \mathrm{K}\right)$

$T_{s}=$ Surface temperature $(\mathrm{K})$

$T_{\infty}=$ Fluid temperature $(\mathrm{K})$

Convection heat transfer can be classified into free, forced or mixed convection depending on the nature of flow. Free convection occurs due to temperature differences in a fluid. These temperature differences cause density differences that interact with gravitational forces and give rise to buoyancy forces which cause the flow. In contrast, forced convection is caused due to external forces like wind or a fan. When convective heat transfer occurs due to both means it is known as mixed convection. In the case of the hotplate there is no external airflow, and therefore this research deals with free convection. 


\subsubsection{Radiation}

Radiation heat transfer is transfer of energy from a surface at a finite temperature. This transfer takes place due to emission of electromagnetic waves from the surface. This transfer between two surfaces takes place without a medium. Hence in vacuum, heat transfer takes place due to radiation. For heat transfer in this research radiation is negligible due to the small size of the hotplates $[9,11]$.

\subsection{Thesis Overview}

Any study of convection is essentially a study (determination) of the parameter $h$. For this research, different values of the convection coefficient $h$ are used in simulations to obtain the resulting temperature of the micro-hotplates due to an applied voltage. These results are then compared with experimental results. The purpose of this research is to obtain the best possible values of $h$ so that the simulated results match the experimental data.

The thesis is divided into five chapters. Chapter one explains the background of the project and work being done. Chapter two gives an explanation of the micro-hotplates on which this work is based. Chapter three covers in detail, the software CoventorWare, which is used for this thesis, including its capabilities and special features. Material properties of the various hotplate materials and the experimental set-up for the work is also covered here. Chapter four and five present the gathered data, results, conclusions, and direction of further research work. 


\section{CHAPTER 2}

\section{DESIGN}

The micro hotplate is a square design suspended by four arms at each corner of the silicon substrate as shown in Figure 1. The white openings are etch holes provided to free the heater structure from the rest of the device. Numerous such hotplate designs can be found in the literature $[3,4,5]$

\subsection{MOSIS Die:}

The original layout is designed in accordance with the MOSIS design rules since devices were to be fabricated through the MOSIS fabrication services [7]. The die size is $2 \mathrm{~mm}$ by $2 \mathrm{~mm}$. The design can fit seventeen (17) hotplates of varying sizes on the die (see Figure 4).

There are three different sizes of hotplates designated here as 'Small', 'Medium' and 'Large'. The basic design of all three sizes is the same except for the different dimensions. There are ten small, four medium and three large hotplates on each chip. Each of the hotplates has two connections for the heater running to the bond pads. One end of all the hotplates are connected together to the 'Ground'(Vss) pad. The other is connected to individual pads, hence allowing control over the operation of individual heaters. 


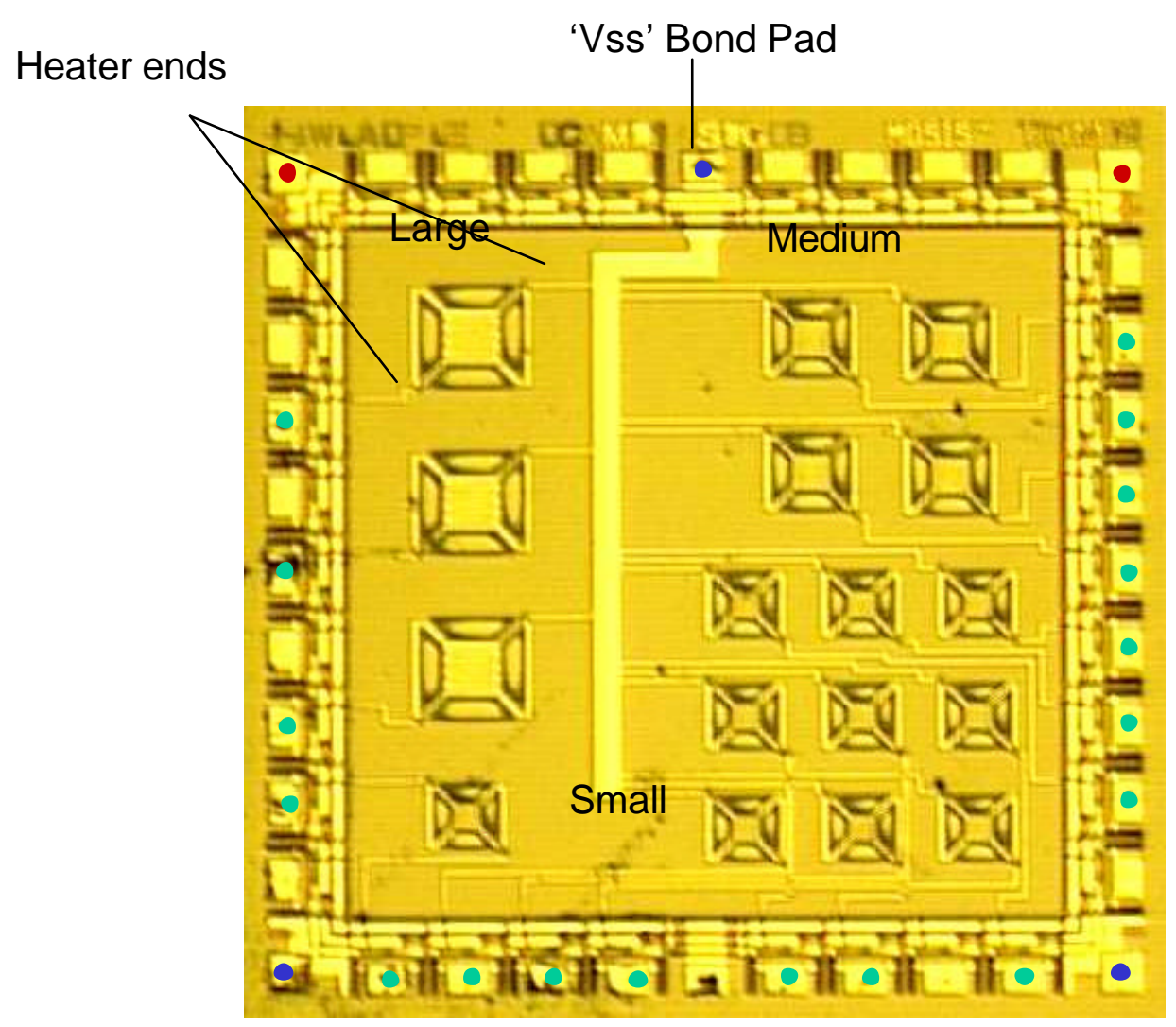

Figure 4: Photograph of actual MOSIS fabricated chip viewed from above (2mm by $2 \mathrm{~mm})[8]$

\subsection{Fabrication:}

Since the devices are fabricated through MOSIS, the layers and materials for the hotplates are dictated by the foundry. There are a total of five layers above the silicon substrate including three oxide layers, a polysilicon and an aluminum layer. The oxide layers are named 'Oxide 0', 'Oxide 1' and 'Oxide 2' with Oxide 0 on the substrate and Oxide 2 at the very top. The heater is made out of polysilicon and sandwiched between Oxide 0 and Oxide 1. An aluminum plate rests above Oxide 1 and finally Oxide 2 . The objective of the aluminum plate is to provide an even distribution of heat over the entire surface. 
The cross-section of the device is shown in Figure 5.

Oxide $2(1.2 \mu \mathrm{m})$

Aluminum $(0.6 \mu \mathrm{m})$

Oxide $1(0.9 \mu \mathrm{m})$

Polysilicon $(0.4 \mu \mathrm{m})$

Oxide $0(0.6 \mu \mathrm{m})$

Substrate

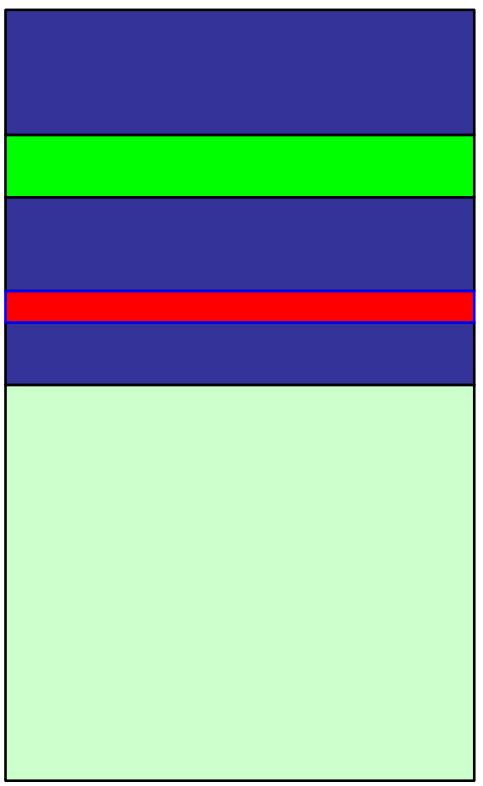

Figure 5: Cross-section of the chip

The material is deposited one by one on top of the substrate and patterned with the appropriate mask. First the oxide is deposited and patterned with the oxide mask then the polysilicon heater, oxide 1 , aluminum and finally oxide 2 . Etch openings are provided through the oxide layers to allow access to the silicon substrate. This allows the etchant (TMAH) to etch away the silicon. The four openings allow the wet etchant to etch under (under-cutting) the center structure until it is completely free from the substrate and is suspended over it.

This type of design isolates the heater from the silicon substrate, which would otherwise dissipate all the heat generated by the heater since silicon is a good thermal conductor. Figure 6 shows a photograph of the actual MOSIS chip after the structure has been released. 


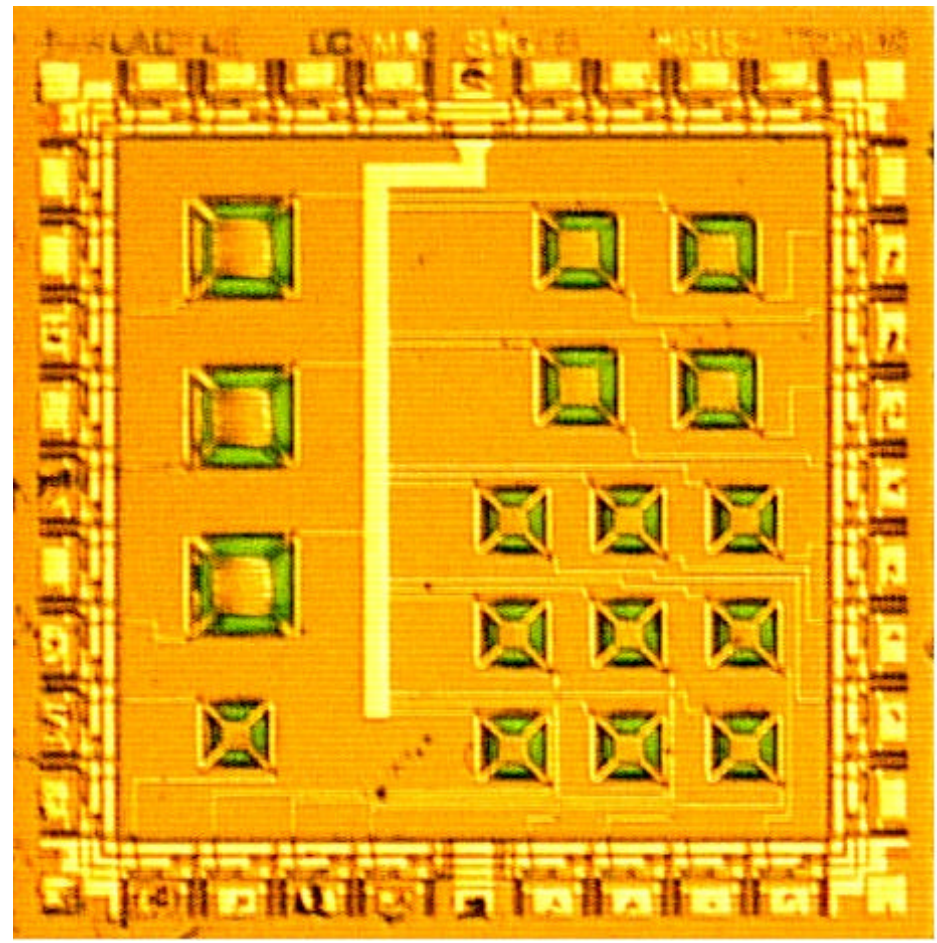

Figure 6: Photograph of actual released micro hotplate device (release procedure performed by Michael Martin) [8].

\subsection{Design Masks and Dimensions:}

As mentioned earlier there are three different sizes of the hotplate on one die. The basic design and materials remain the same for all the layers for all the sizes. The changes are in the dimensions of the components; the hotplate sizes are scaled, however the scaling is uneven. Figures 7,8 and 9 show the three components of the micro hotplate. Each element of the component is defined and the corresponding measurement noted in Table 1. The devices are compared in Table 1 which gives a better idea of the size difference. As shown in Figures 3 and 8 , the aluminum plate rests on two sides of the substrate. These two arm widths are $14 \mu \mathrm{m}, 15 \mu \mathrm{m} \& 15 \mu \mathrm{m}$ respectively for small, medium and large hotplates. 


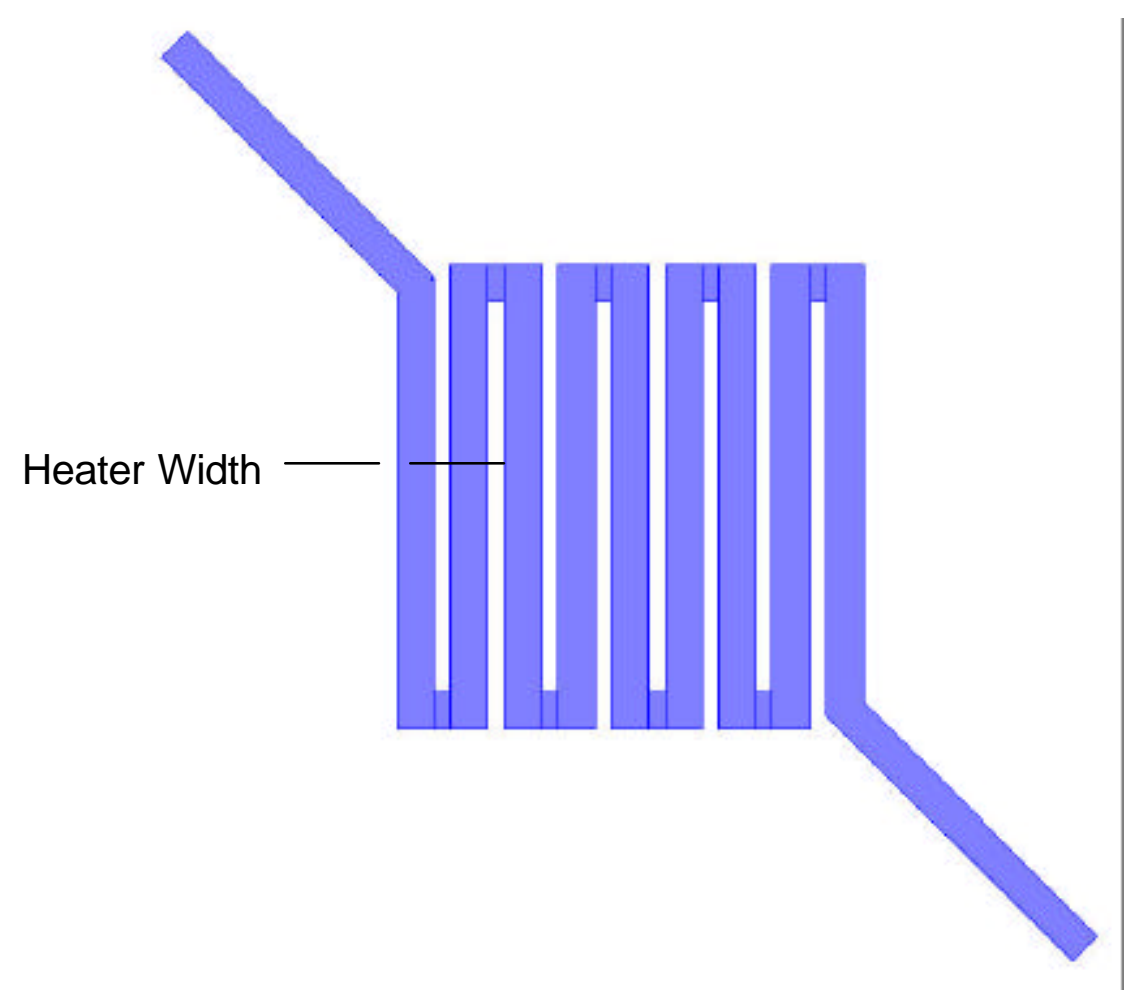

Figure 7: Mask design for the polysilicon heater.

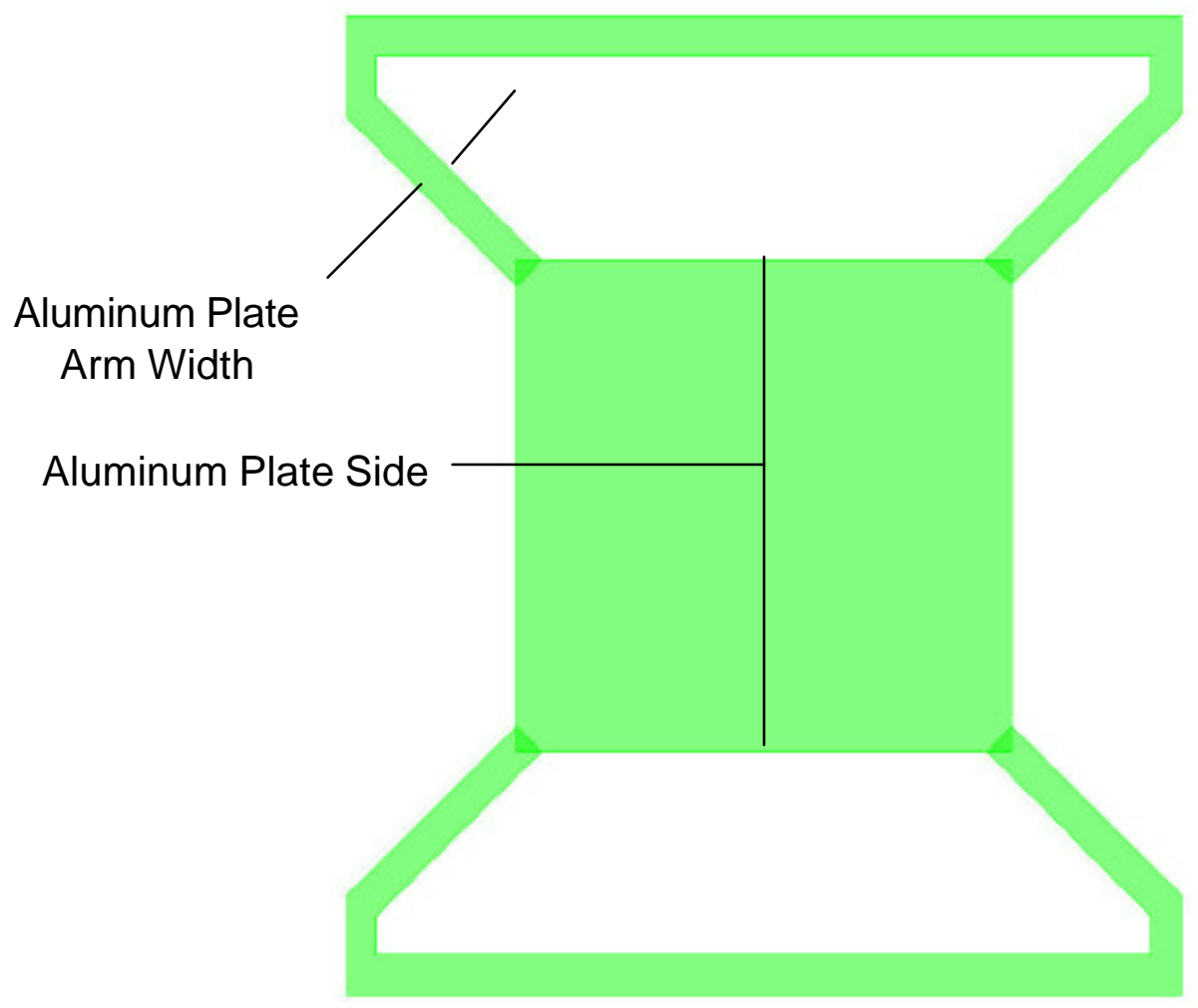

Figure 8: Mask design for the aluminum distribution plate. 


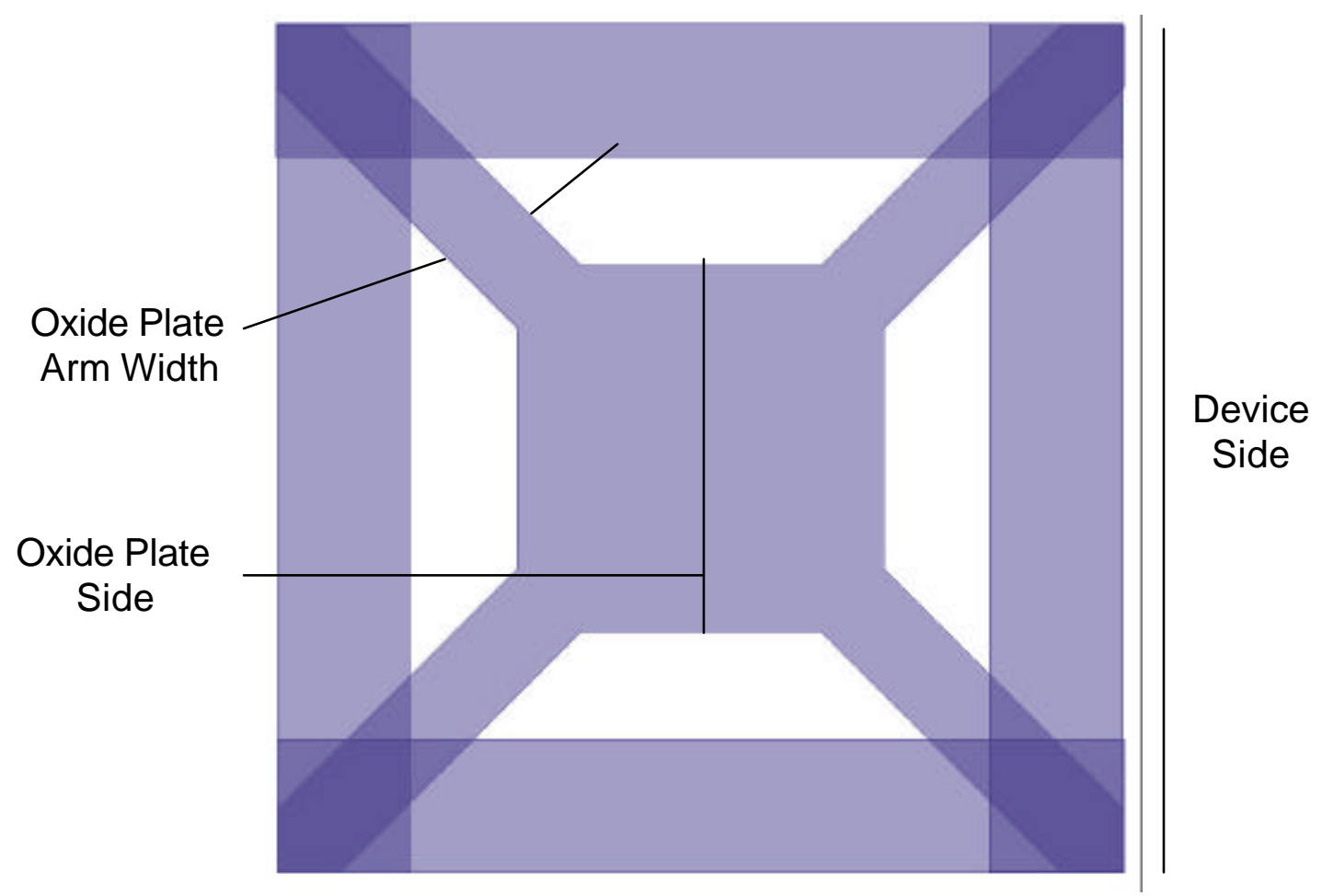

Figure 9: Mask design for the oxide layer.

Table 1:

Dimensions of the hotplate components

\begin{tabular}{|c|c|c|c|}
\hline Dimensions $(\mu \mathrm{m})$ & Small & Medium & Large \\
\hline Heater Width & 8 & 9 & 14 \\
\hline Aluminum Plate Side & 90 & 123 & 186 \\
\hline Aluminum Plate Arm Width & 14.1 & 12 & 13 \\
\hline Oxide Plate Side & 100 & 133 & 198 \\
\hline Oxide Plate Arm Width & 28 & 33.94 & 48 \\
\hline Device Side & 332 & 373 & 458 \\
\hline
\end{tabular}


Figure 10 shows all the three hotplates on one scale. The size difference is more evident and the uneven scaling is noticeable.
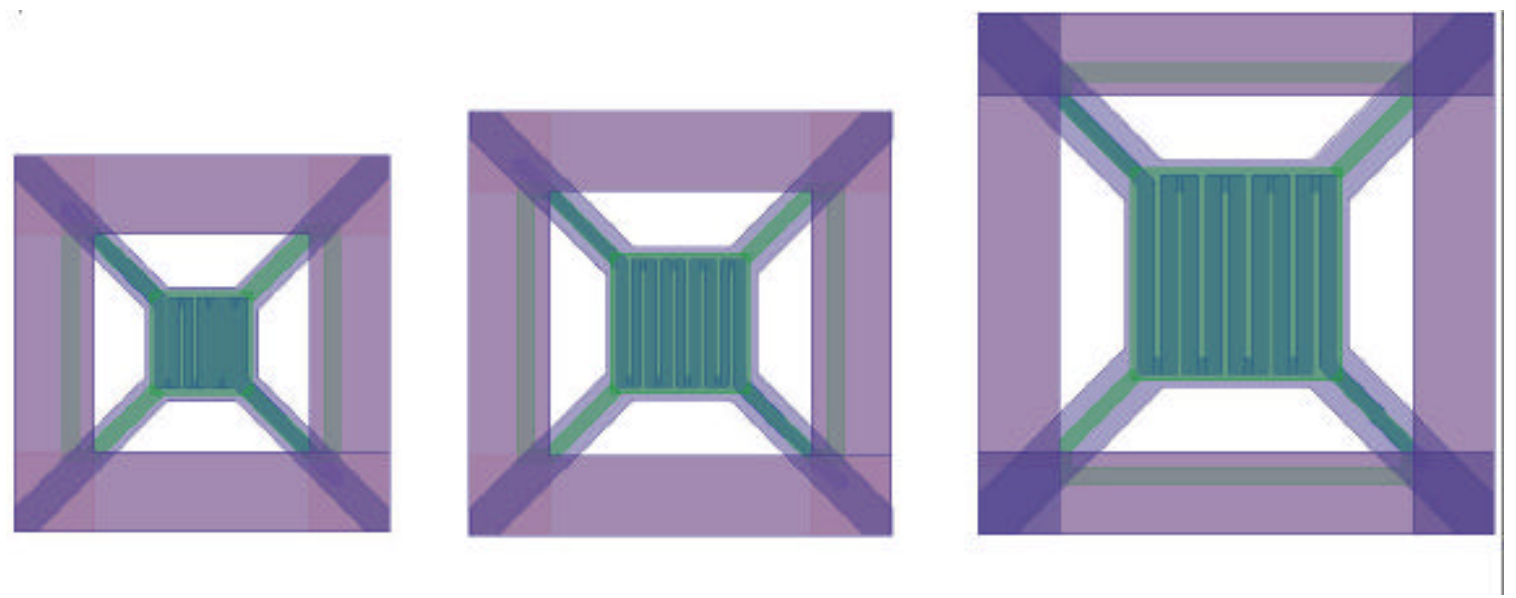

Figure 10: Design layouts of all three hotplates. 


\section{CHAPTER 3}

\section{MODELING}

\subsection{CoventorWare ${ }^{\mathrm{TM}}$ (MEMCAD)}

Computer tools have been used to aid the design and modeling for various different hotplate structures, common examples being MAGIC [4], ANSYS, etc. Finite Element Modeling (FEM) has been used for quite a while $[9,10]$ and modern software feature tools which utilize FEM to simulate MEMS devices [11].

The computer software used for the simulations of the hotplates for this research is known as CoventorWare, formerly known as MEMCAD. The software is designed and released by Coventor, hence the name. It is a powerful tool for the MEMS field with capabilities ranging from designing layouts, mask generation, FEM modeling and simulations for MEMS and microfluidic devices. The version used for this research work is CoventorWare2003, one of two licensed versions available at the University of Louisville.

\subsection{Software Reliability}

When using a software for research work, there is always reasonable doubt of the capabilities and reliability of the results produced by the software. To optimize CoventorWare for research, a small project was undertaken before proceeding with the research.

CoventorWare has been used to run simulations on microhotplate devices by a few groups around the world. D. Briand et. al. [11, 12 ] have successfully used 
this software to simulate their device and then corroborated the results obtained with experimental data thus proving the accuracy of CoventorWare. The project involved recreating the same device in the software and simulating it using the same boundary conditions.

Figure 11 shows the results from the paper while Figure 12 shows the results from the project. There is a small difference of $10^{\circ} \mathrm{K}$ between the two results. This is due to the fact that the recreated design isn't exactly the same as the original one (due to limited design information). However the results prove sufficiently that the software can be used effectively and reliably for modeling and simulation. 


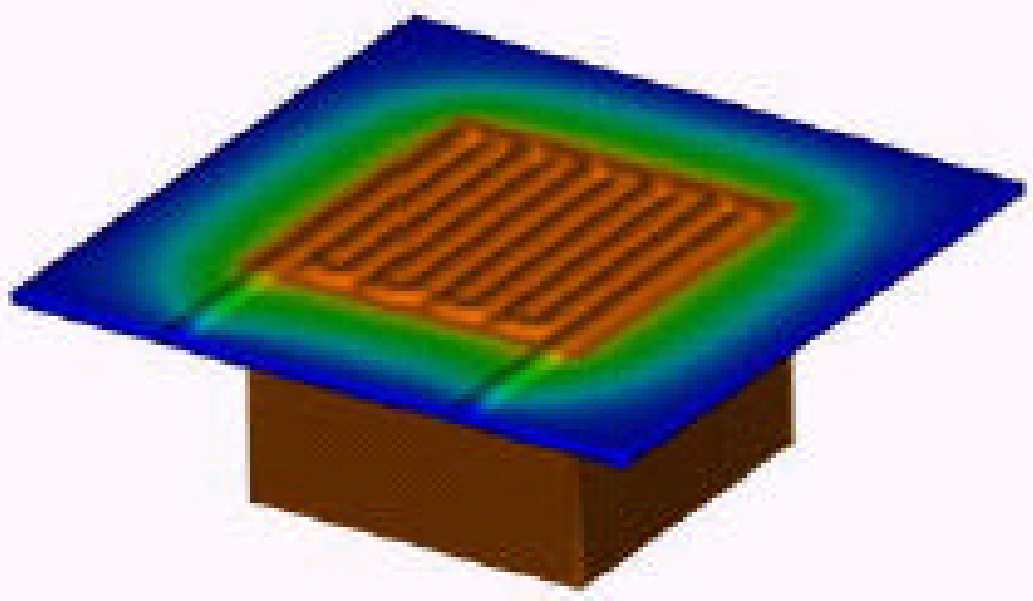

Temperature $[\mathrm{k}]$

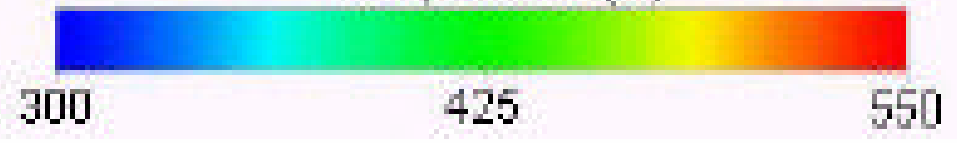

Figure 11: Simulation results of original design [11].

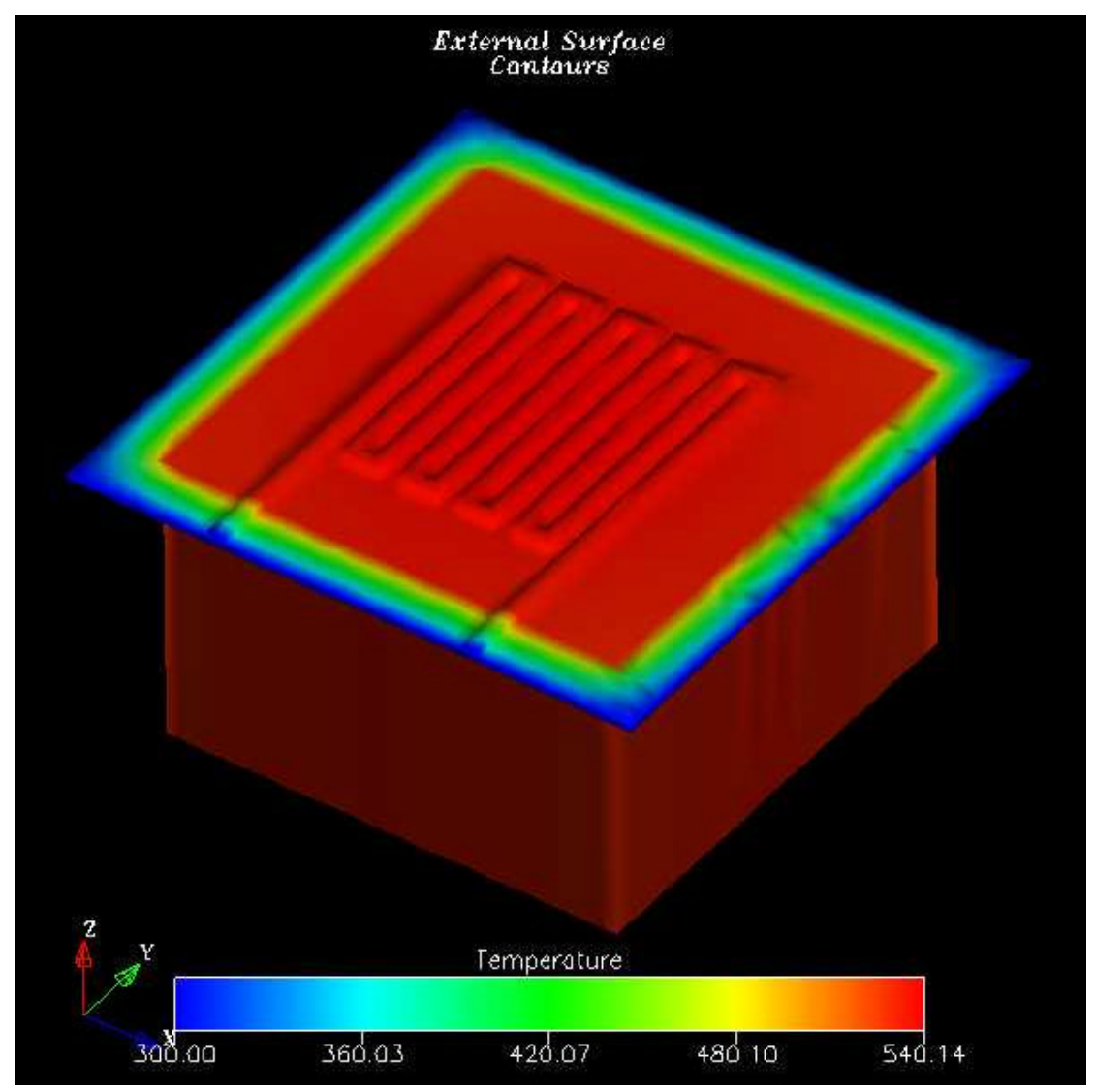

Figure 12: Simulation results of recreated device. 


\subsection{2-D Designing}

CoventorWare2003 has two setups to choose from on start-up; MEMS or Microfluidics. This research deals with MEMS devices which is further divided into three distinct sections, ARCHITECTTM [13], DESIGNER ${ }^{\mathrm{TM}}$ [14] and ANALYZERTM $[15,16 \& 17]$. ARCHITECTTM deals with system level approach for simulations. It uses behavioral models and parametric libraries to simulate system behavior. This work involves use of DESIGNER ${ }^{\mathrm{TM}}$ and ANALYZER ${ }^{\mathrm{TM}}$ for physical behavior of the device.

The DESIGNER ${ }^{\mathrm{TM}}$ module has the capability to design 2-D models from scratch, importing layouts designed in other software and storing and exporting in various formats. Since the original layout was designed in L-Edit ${ }^{T M}$, the design files are stored in the '.tdb' format. Since CoventorWare doesn't recognize this format, the files were exported in the '.gds' and '.cif' formats from L-Edit'TM. These are the most common file types used for mask generation. The design files were imported into CoventorWare and then converted and stored in the local format '.cat'. These 2D layout files provide the necessary ' $x y$ ' coordinates information to the software.

\subsection{3-D Model, Meshing and Simulation}

All the layer information is entered in a process file. The process file stores information such as material, layer thickness, deposition techniques, sidewall angles, etc. Figure 13 shows the step-by-step processes required for building the 3-D model of the MOSIS hotplates in CoventorWare. 
The process file provides the software with the information to calculate the ' $z$ ' co-ordinates for building the solid model. Since MEMS devices are built from layers of uniform thickness deposited over the surface, only the thickness of the layers is required for a 3-D model. The software creates a 3-D model (Figure 14) using the 2-D layout files and this process file.

\begin{tabular}{|c|c|c|c|c|c|c|c|c|c|c|c|c|c|}
\hline Step & Action & Type & $\begin{array}{l}\text { Layer } \\
\text { Name }\end{array}$ & Material & Thickne & & Color & $\begin{array}{l}\text { Mask Namer } \\
\text { Polarity }\end{array}$ & & Depth & Offset & $\begin{array}{l}\text { Sidewall } \\
\text { Angle }\end{array}$ & Comment \\
\hline 0 & Base & & Substrate & SILICON & 50,0 & & blue & GND & & & & & \\
\hline 1 & Etch & Back, Substrate & & & & & cyan & substrate & + & 50.0 & 0.0 & 0.0 & \\
\hline 2 & Deposit & Planar & sacr & BPSG & 0.0 & & red & & & & & & \\
\hline 3 & Deposit & Stacked & oxide0 & OXIDE & 0.6 & & gold & & ; & in & רו" & 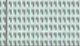 & \\
\hline 4 & Etch & Front, Last Layer & & & & & cyan & oxide & + & 0.6 & 0.0 & 0.0 & \\
\hline 5 & Deposit & Stacked & heater & Polysilicon_MOSIS & 0.4 & & green & (3) & in & ; & 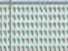 & 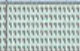 & \\
\hline 6 & Elch & Front, Last Layer & & & & & white & heater & + & 0.4 & 0.0 & 0.0 & \\
\hline 7 & Deposit & Conformal & oxide! & OXIDE & 0,9 & SCF & gold & & ….......... & & הan & 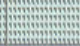 & \\
\hline 8 & Etch & Front, Last Layer & 灌 & & & & cyan & oxide & + & 0.9 & 0.0 & 0.0 & \\
\hline 9 & Deposit & Conformal & alum & ALLIMINUM(FILM) & 0.6 & SCF & gold & & 兴 & ;., & 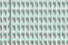 & 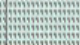 & \\
\hline 10 & Etch & Front, Last Layer & & & & & cyan & alum & + & 0.6 & 0.0 & 0.0 & \\
\hline 11 & Deposit & Conformal & oxide2 & OXIDE & 1.2 & SCF & gold & & in. & ;in, & 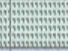 & ; & \\
\hline 12 & Etch & Front, Last Layer & & & & & cyan & oxide & + & 1.2 & 0.0 & 0.0 & \\
\hline 13 & Sacrifice & & & BPSG & & & & & & & & & \\
\hline
\end{tabular}

Figure 13: The CoventorWare process file for the hotplates.

The process file allows for one deposition step to be followed by an etch step for that layer. This is not necessarily true for actual fabrication processes. The substrate etch is the last step in actual fabrication while the software requires it to be etched immediately after deposition. CoventorWare is not able to simulate all possible fabrication processes, such as the final TMAH anisotropic etch that frees the hotplate. However, CoventorWare is flexible enough that it is usually possible to build a 3-D model of the desired device using unique combinations of the process steps included in the software. 


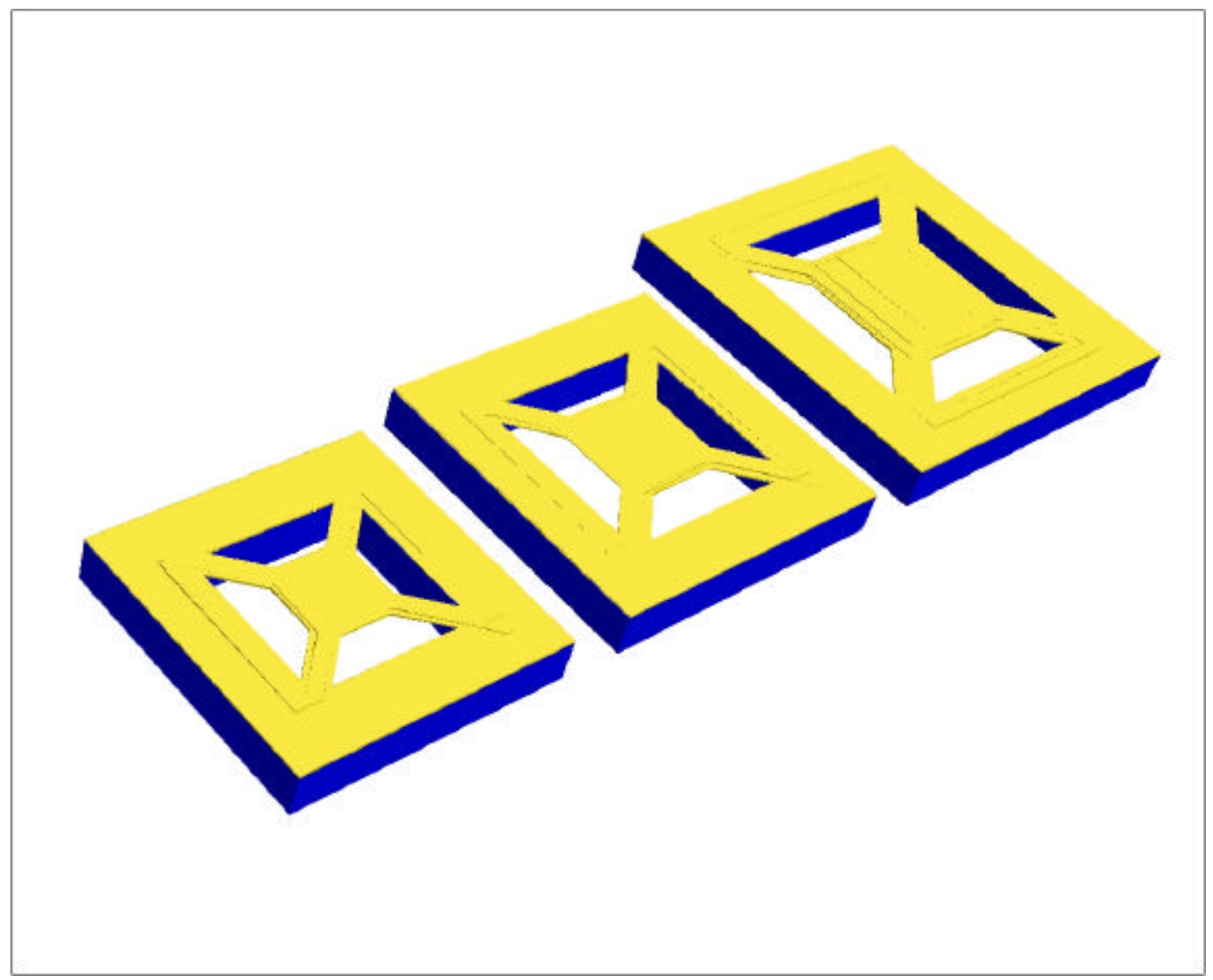

Figure 14: 3-D solid models of the micro-hotplates.

For example, in this case it was required to include a sacrificial layer with '0' thickness to be deposited, after the substrate etch, to obtain the correct model (see Figure 14).

After the model has been obtained, the next step is to generate a mesh. CoventorWare uses finite element and boundary element analysis techniques for its simulations. Meshing involves division of the 3-D model into a number of small elements each with a specified number of nodes. For the ElectroThermal Solver the mesher setting is 'Extruded Bricks' wherein the top surfaces of the layers are 
imprinted into each other. The imprinted faces are then meshed using the 'Split and Merge' quadrilateral meshing algorithm which is then extruded through the ' $z$ ' direction to generate the final Hexahedral mesh (see Figures 15, 16, \& 17).

\subsection{Experimental Data}

The experimental data for this research work have been obtained from the work done by Dr. Huey-Daw Wu [8] at the Naval Research Laboratory, Washington D.C. The MOSIS chips were etched at UofL (by Michael Martin) and sent to NRL for characterization (by Dr. Huey-Daw Wu). Two separate experiments were performed on the devices; the first one consisted of obtaining Voltage-Current-Resistance (V-I-R) data while the second experiment involved heating the devices to obtain Temperature-Resistance (T-R) data.

\subsubsection{First Experiment}

For the V-I-R measurements, a Keithley SourceMeter was used in conjunction with precision DC probes. The voltage is varied from $0 \mathrm{~V}$ to $15 \mathrm{~V}$ in steps of $1 \mathrm{~V}$ each and the corresponding current through the heaters is measured (Table 5 and Figure 18).

Using Ohm's law, the resistance of the heaters is calculated for each step (see Table 6 and Figure19).

$$
V=I R
$$

Similarly using the equation;

$$
\mathrm{P}=\mathrm{VI}
$$




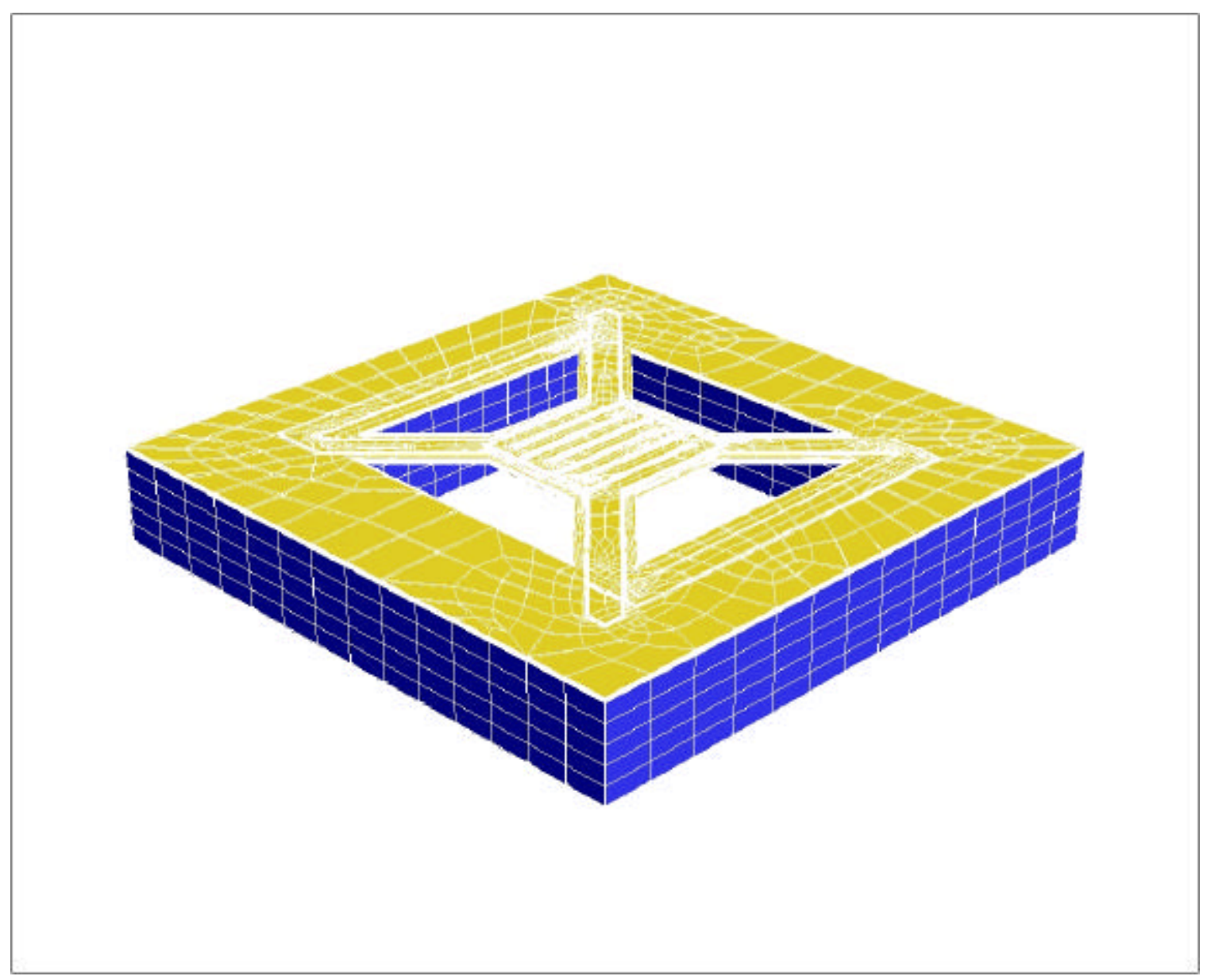

Figure 15: Mesh for the small hotplate.

Table 2: Mesher settings for Small hotplate.

\begin{tabular}{|c|c|c|}
\hline Layer & xy $(\mu \mathrm{m})$ & $\mathrm{z}(\mu \mathrm{m})$ \\
\hline Substrate & 25 & 10 \\
\hline Oxide 0 & 25 & 0.3 \\
\hline Heater & 8 & 0.2 \\
\hline Oxide 1 & 25 & 0.45 \\
\hline Aluminum & 25 & 0.3 \\
\hline Oxide 2 & 25 & 0.6 \\
\hline
\end{tabular}




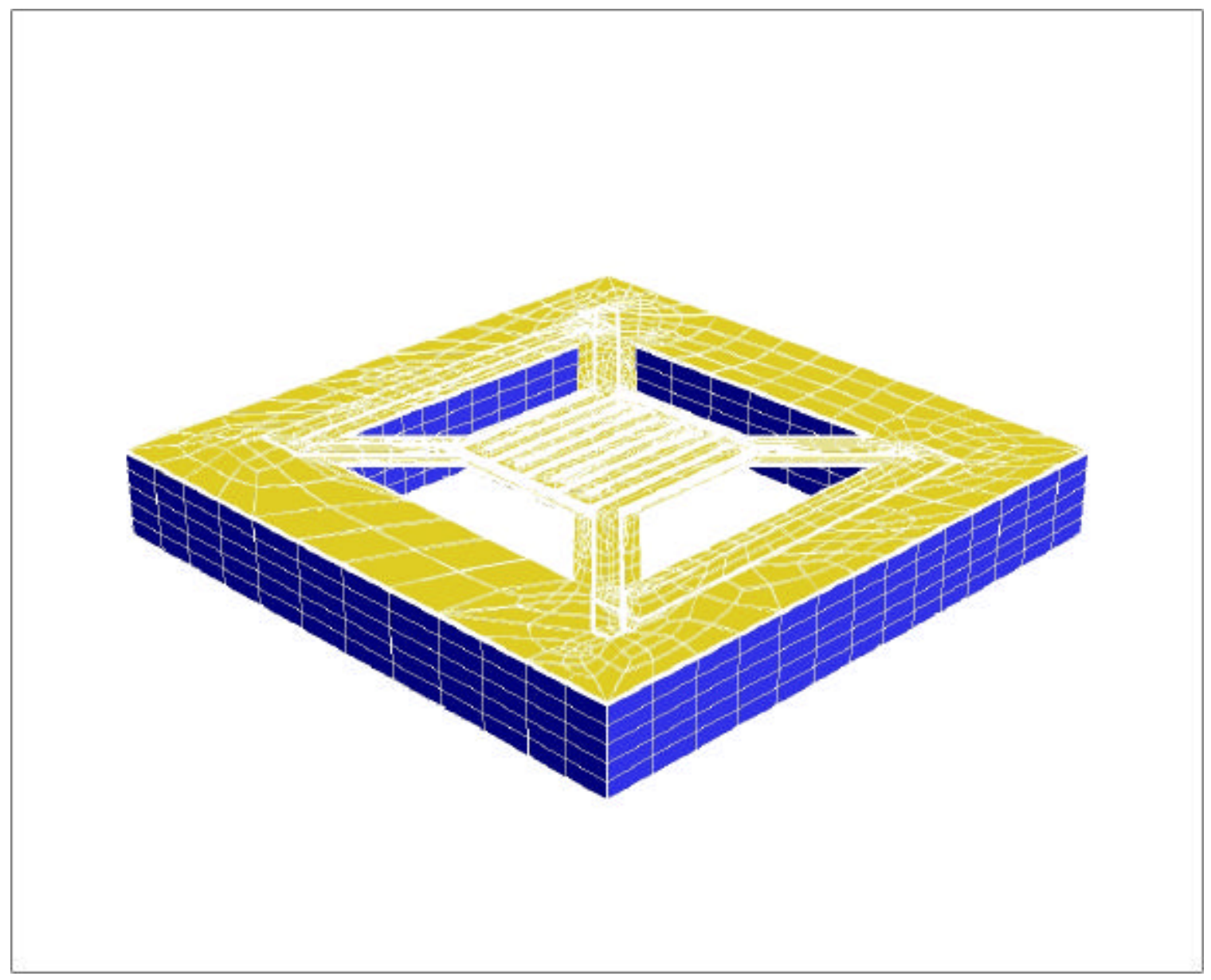

Figure 16: Mesh for the medium hotplate.

Table 3: Mesher settings for Medium hotplate.

\begin{tabular}{|c|c|c|}
\hline Layer & xy $(\mu \mathrm{m})$ & $\mathrm{z}(\mu \mathrm{m})$ \\
\hline Substrate & 35 & 10 \\
\hline Oxide 0 & 35 & 0.3 \\
\hline Heater & 9 & 0.2 \\
\hline Oxide 1 & 35 & 0.35 \\
\hline Aluminum & 35 & 0.6 \\
\hline Oxide 2 & 35 & \\
\hline
\end{tabular}




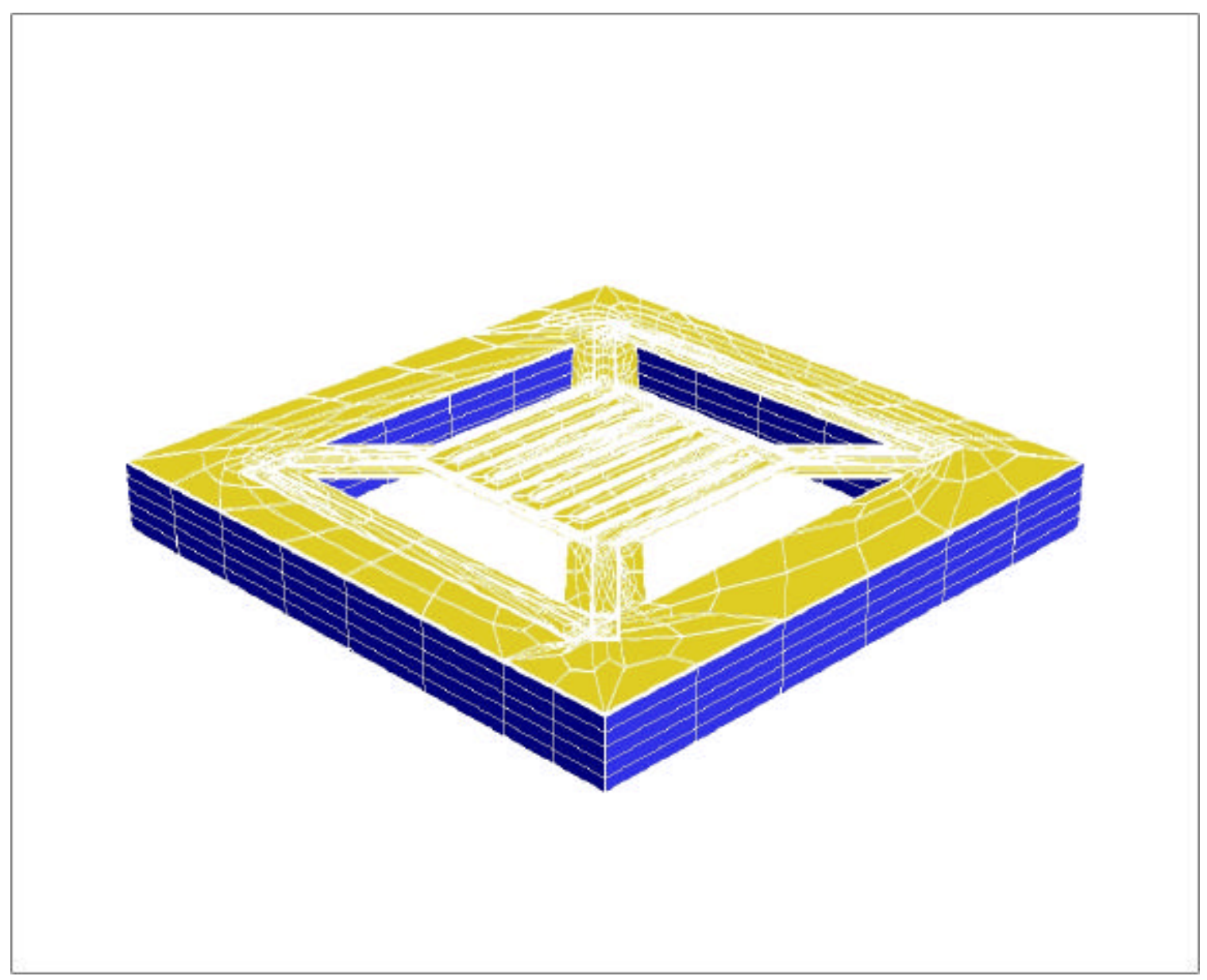

Figure 17: Mesh for the large hotplate.

Table 4: Mesher settings for Large hotplate.

\begin{tabular}{|c|c|c|}
\hline Layer & xy $(\mu \mathrm{m})$ & $\mathrm{z}(\mu \mathrm{m})$ \\
\hline Substrate & 65 & 10 \\
\hline Oxide 0 & 65 & 0.3 \\
\hline Heater & 13 & 0.2 \\
\hline Oxide 1 & 65 & 0.45 \\
\hline Aluminum & 65 & 0.6 \\
\hline Oxide 2 & 65 & \\
\hline
\end{tabular}


power can be calculated where power $=\mathrm{P}$ is the product of the applied voltage and the resulting current. The results are tabulated in Table 7 and shown graphically in Figure 20.

\subsubsection{Second Experiment}

For the second experiment, a Thermal Probe Station is used to heat the devices and measure the heater resistances at appropriate steps. For this the chips are mounted on a Polyimide sheet with an opening large enough to fit the die. The device is then placed inside a thermal probe station where the chip is in direct contact with the thermal chuck. The temperature is varied from $20^{\circ} \mathrm{C}$ to $200^{\circ} \mathrm{C}$ and the resistance of the heater is measured in steps of $10^{\circ} \mathrm{C}$ (see Table 8).

For measuring the resistance, a sense current has to be passed through the heater from the SourceMeter. This sense current is kept fixed at $10 \mu \mathrm{A}$ to prevent the heaters from heating up and causing errors in the data. This ensures that the only source of heat in the chamber is from the thermal chuck.

As seen from Tables 5, 6, \& 7 and Figures $18,19 \& 20$, the medium hotplate has the highest resistance amongst the three and hence the lowest current and power for the same voltage. Similarly, in Table 8 it is seen that the data is uneven, in that it does not scale according to the size. The resistance of the large hotplate is greater than the small hotplate by more than $1 \mathrm{KO}$ at all temperatures. The resistance values of the medium hotplate are slightly greater than those for the large ones which is unusual since the expected values would be between those of the small and the large hotplates (see Figure 21). 
These high resistance values cause the medium hotplate to heat up to higher temperatures than the other two sizes for the same power (Table 10 \& Figure 23). These values are calculated and deduced from Tables $7 \& 9$.

\section{Table 5:}

Measured Current (mA) vs. Voltage $(\mathrm{V})$ data

\begin{tabular}{|c|c|c|c|}
\hline \multirow[t]{2}{*}{ Voltage (V) } & \multicolumn{3}{|c|}{ Current $(m A)$} \\
\hline & Small & Medium & Large \\
\hline 0 & 0 & 0 & 0 \\
\hline 1 & 0.40088 & 0.26043 & 0.27422 \\
\hline 2 & 0.79602 & 0.51777 & 0.5458 \\
\hline 3 & 1.1805 & 0.76944 & 0.81266 \\
\hline 4 & 1.5493 & 1.0126 & 1.0722 \\
\hline 5 & 1.8971 & 1.2441 & 1.3217 \\
\hline 6 & 2.2228 & 1.4636 & 1.5608 \\
\hline 7 & 2.5226 & 1.6689 & 1.7876 \\
\hline 8 & 2.7953 & 1.8587 & 2.0007 \\
\hline 9 & 3.0431 & 2.0338 & 2.2004 \\
\hline 10 & 3.2655 & 2.1941 & 2.3859 \\
\hline 11 & 3.4639 & 2.3401 & 2.5577 \\
\hline 12 & 3.6408 & 2.473 & 2.717 \\
\hline 13 & 3.7969 & 2.5931 & 2.8632 \\
\hline 14 & 3.9341 & 2.7016 & 2.9978 \\
\hline 15 & 4.0546 & 2.7997 & 3.1214 \\
\hline
\end{tabular}


Table 6:

Calculated Resistance (O) vs. Voltage (V) data

\begin{tabular}{|c|c|c|c|}
\hline \multirow{2}{*}{$\begin{array}{l}\text { Voltage } \\
\text { (V) }\end{array}$} & \multicolumn{3}{|c|}{ Resistance (O) } \\
\hline & Small & Medium & Large \\
\hline 0 & 2489 & 3833 & 3641 \\
\hline 1 & 2494.5 & 3839.9 & 3646.7 \\
\hline 2 & 2512.5 & 3862.7 & 3664.3 \\
\hline 3 & 2541.2 & 3898.9 & 3691.6 \\
\hline 4 & 2581.7 & 3950.3 & 3730.7 \\
\hline 5 & 2635.5 & 4019 & 3783.1 \\
\hline 6 & 2699.3 & 4099.6 & 3844.2 \\
\hline 7 & 2774.9 & 4194.3 & 3915.9 \\
\hline 8 & 2861.9 & 4304.1 & 3998.6 \\
\hline 9 & 2957.5 & 4425.1 & 4090.1 \\
\hline 10 & 3062.3 & 4557.8 & 4191.3 \\
\hline 11 & 3175.6 & 4700.6 & 4300.7 \\
\hline 12 & 3295.9 & 4852.3 & 4416.7 \\
\hline 13 & 3423.9 & 5013.4 & 4540.3 \\
\hline 14 & 3558.6 & 5182.2 & 4670.1 \\
\hline 15 & 3699.5 & 5357.7 & 4805.5 \\
\hline
\end{tabular}




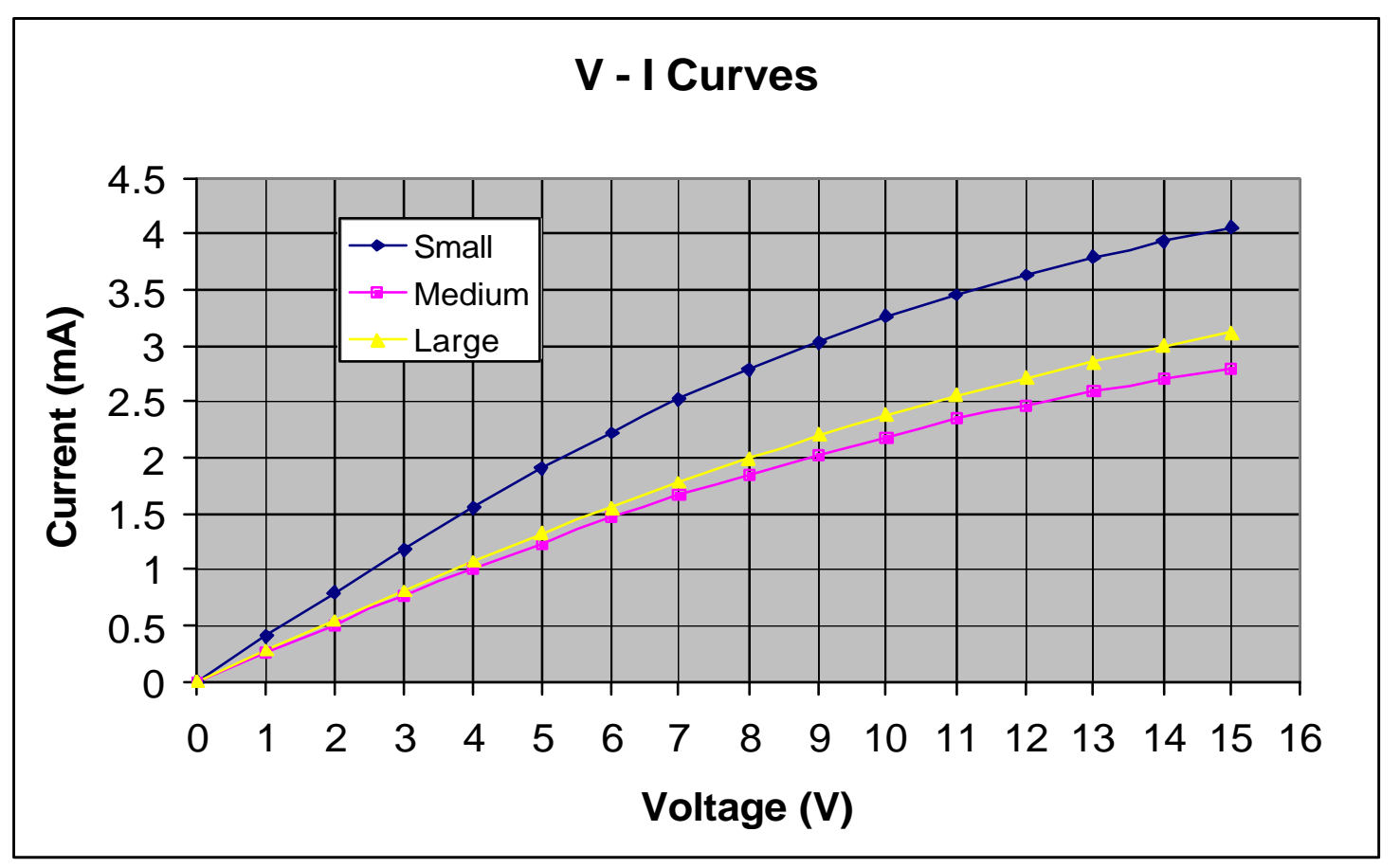

Figure 18: Measured Current (mA) vs. Voltage (V) curves.

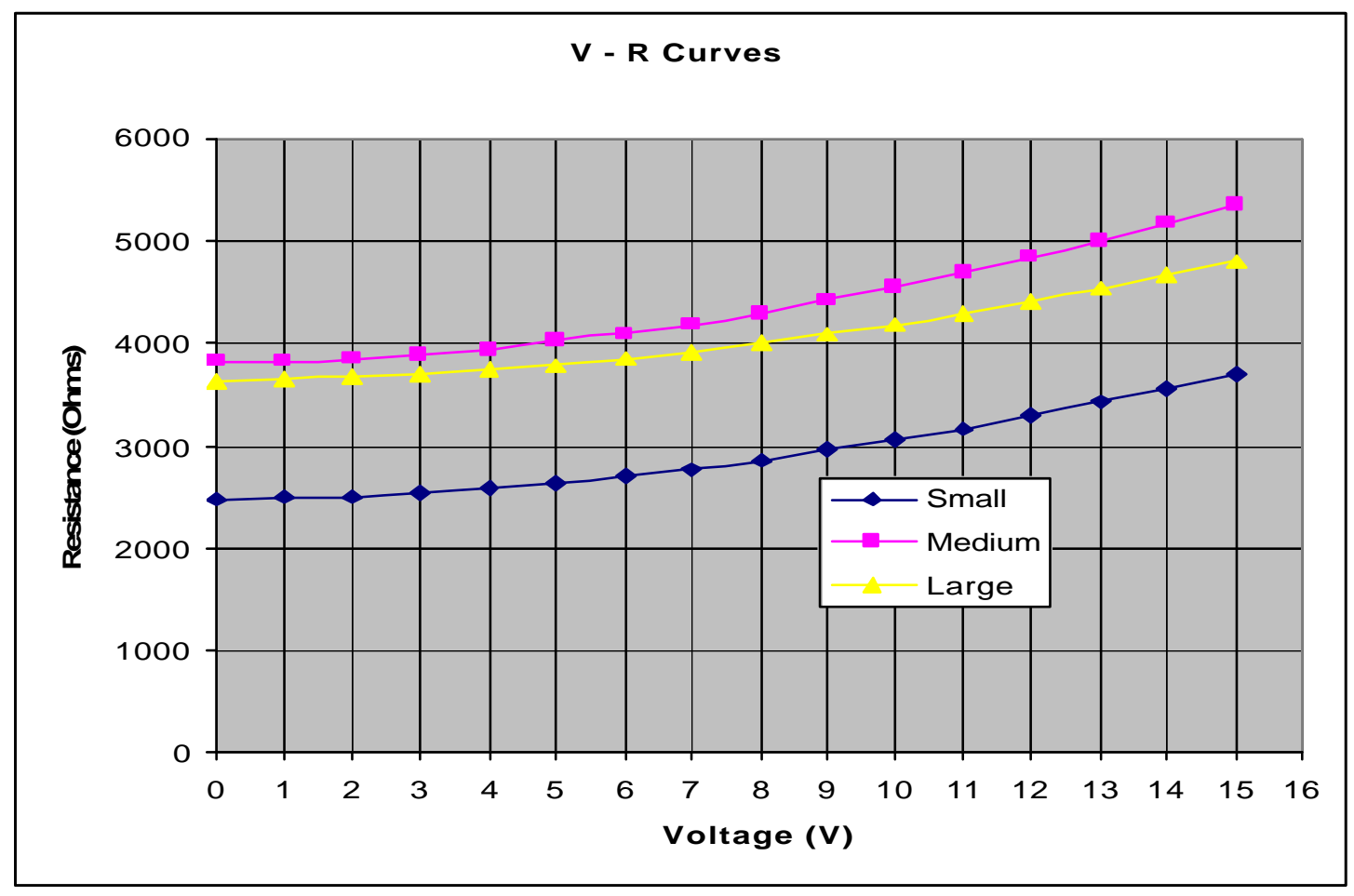

Figure 19: Calculated Resistance $(\mathrm{O})$ vs. Voltage (V) curves. 
Table 7:

Calculated Power (mW) vs. Voltage(V) data

\begin{tabular}{|c|c|c|c|}
\hline \multirow{2}{*}{ Voltage (V) } & \multicolumn{3}{|c|}{ Power (mW) } \\
\hline & Small & Medium & Large \\
\hline 0 & 0 & 0 & 0 \\
\hline 1 & 0.4009 & 0.26043 & 0.2742 \\
\hline 2 & 1.592 & 1.0355 & 1.0916 \\
\hline 3 & 3.5416 & 2.3083 & 2.438 \\
\hline 4 & 6.1974 & 4.0503 & 4.2887 \\
\hline 5 & 9.4857 & 6.2204 & 6.6084 \\
\hline 6 & 13.3366 & 8.7814 & 9.3648 \\
\hline 7 & 17.6581 & 11.6826 & 12.5132 \\
\hline 8 & 22.3628 & 14.8697 & 16.0056 \\
\hline 9 & 27.3882 & 18.3045 & 19.8039 \\
\hline 10 & 32.6549 & 21.9405 & 23.8588 \\
\hline 11 & 38.1032 & 25.7415 & 28.1351 \\
\hline 12 & 43.6901 & 29.6765 & 32.6035 \\
\hline 13 & 49.3591 & 33.7097 & 37.2219 \\
\hline 14 & 55.0775 & 37.822 & 41.9693 \\
\hline 15 & 60.819 & 41.9954 & 46.8212 \\
\hline
\end{tabular}




\section{Table 8:}

Measured Resistance $(\mathrm{O})$ vs. Temperature $\left({ }^{\circ} \mathrm{C}\right)$ data (thermal chuck used to control temperature and resistance measured at a sense current of $10 \mu \mathrm{A}$ to minimize self-heating effects).

\begin{tabular}{|c|c|c|c|}
\hline \multirow{2}{*}{$\begin{array}{l}\text { Temperature } \\
\qquad\left({ }^{\circ} \mathrm{C}\right)\end{array}$} & \multicolumn{3}{|c|}{ Resistance (O) } \\
\hline & Small & Medium & Large \\
\hline 25 & 2489 & 3833 & 3641 \\
\hline 30 & 2500 & 3849.5 & 3656.7 \\
\hline 40 & 2522.5 & 3883 & 3689 \\
\hline 50 & 2545.5 & 3917 & 3722.3 \\
\hline 60 & 2568.8 & 3951.4 & 3756 \\
\hline 70 & 2592.4 & 3986 & 3790 \\
\hline 80 & 2616.3 & 4021 & 3824 \\
\hline 90 & 2640.4 & 4056 & 3858 \\
\hline 100 & 2664.6 & 4091 & 3892 \\
\hline 110 & 2689 & 4126 & 3926 \\
\hline 120 & 2713.5 & 4161 & 3960 \\
\hline 130 & 2738.2 & 4196 & 3994 \\
\hline 140 & 2763 & 4231 & 4028 \\
\hline 150 & 2788 & 4266 & 4062 \\
\hline 160 & 2813 & 4301 & 4096 \\
\hline 170 & 2838 & 4336 & 4129.7 \\
\hline 180 & 2863 & 4370.5 & 4163 \\
\hline 190 & 2888 & 4405 & 4196 \\
\hline 200 & 2913 & 4439 & 4229 \\
\hline
\end{tabular}




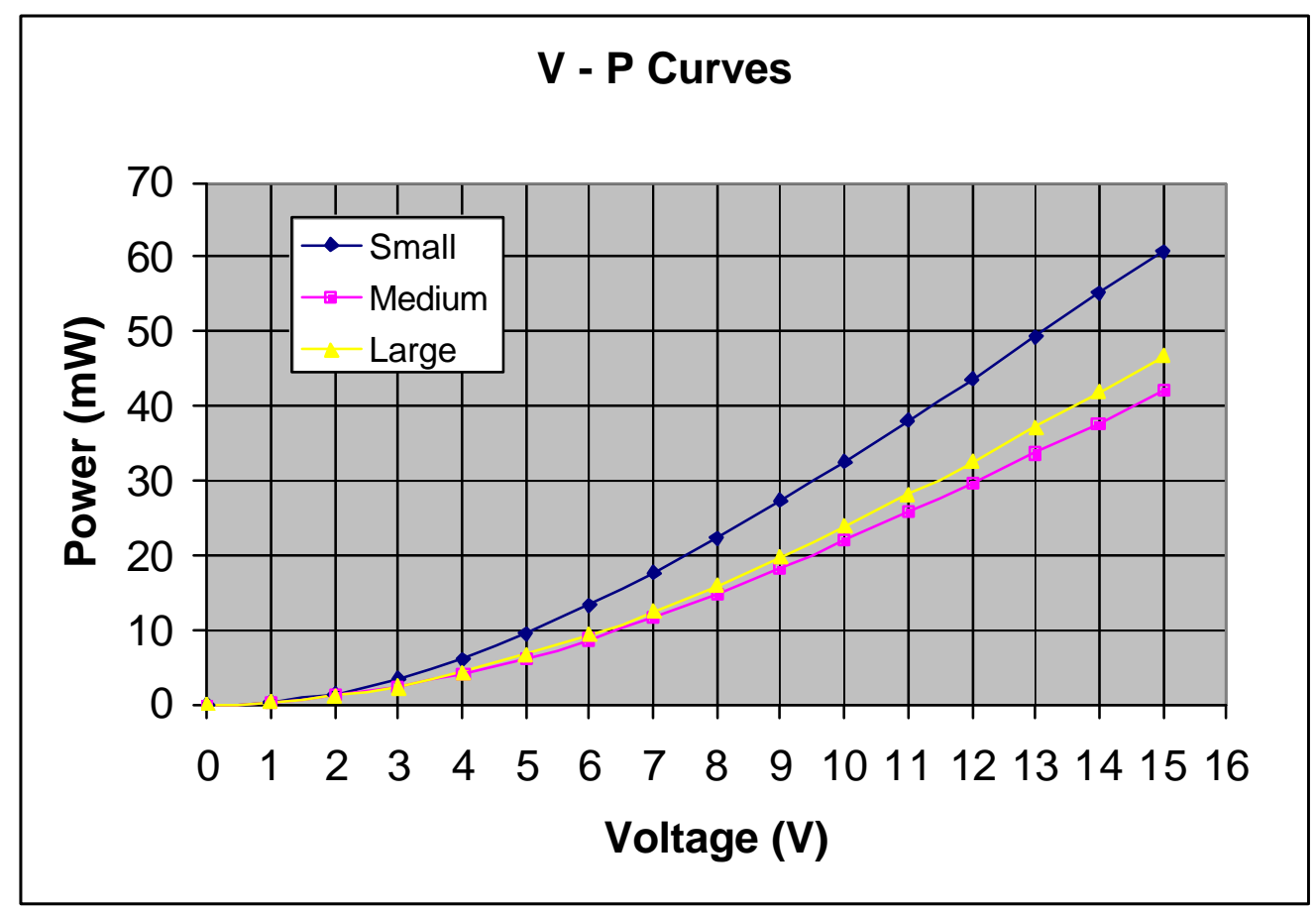

Figure 20: Calculated Power (mW) vs. Voltage (V) curves.

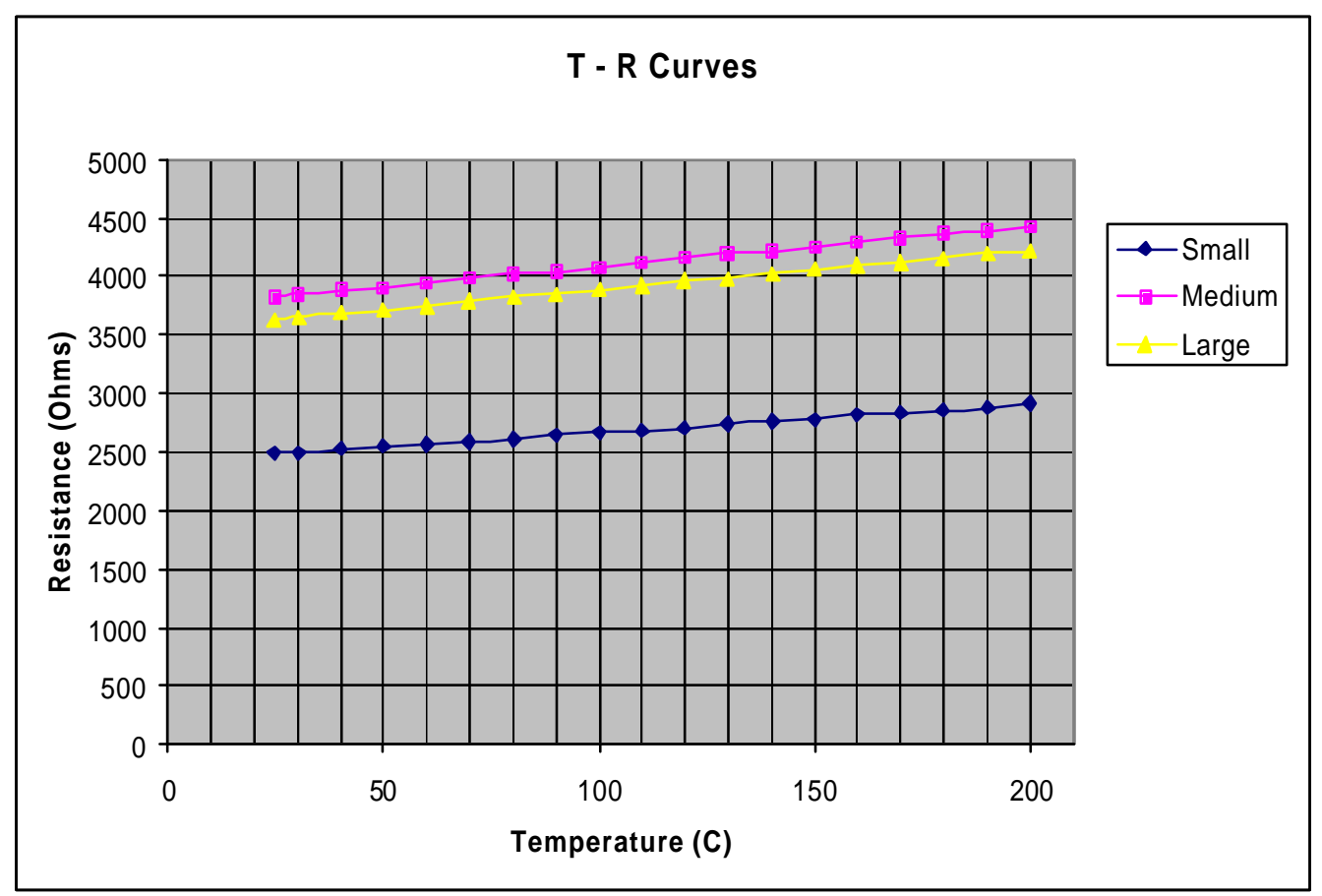

Figure 21: Measured Resistance $(\mathrm{O})$ vs. Temperature $\left({ }^{\circ} \mathrm{C}\right)$ curves (thermal chuck used to control temperature) 
Table 9:

Deduced Temperature $\left({ }^{\circ} \mathrm{C}\right)$ vs. Voltage $(\mathrm{V})$ data

\begin{tabular}{|c|c|c|c|}
\hline \multirow{2}{*}{$\begin{array}{l}\text { Voltage } \\
\text { (V) }\end{array}$} & \multicolumn{3}{|c|}{ Temperature (C) } \\
\hline & Small & Medium & Large \\
\hline 0 & 25 & 25 & 25 \\
\hline 1 & 27.296 & 26.98 & 26.7 \\
\hline 2 & 34.809 & 33.587 & 31.94 \\
\hline 3 & 46.8 & 44.055 & 40.04 \\
\hline 4 & 63.731 & 58.911 & 51.69 \\
\hline 5 & 86.198 & 78.77 & 67.26 \\
\hline 6 & 112.836 & 102.053 & 85.45 \\
\hline 7 & 144.403 & 129.422 & 106.77 \\
\hline 8 & 180.722 & 161.151 & 131.38 \\
\hline 9 & 220.635 & 196.144 & 158.61 \\
\hline 10 & 264.422 & 234.481 & 188.72 \\
\hline 11 & 311.717 & 275.756 & 221.25 \\
\hline 12 & 361.978 & 319.615 & 255.77 \\
\hline 13 & 415.408 & 366.17 & 292.55 \\
\hline 14 & 471.673 & 414.947 & 331.15 \\
\hline 15 & 530.505 & 465.691 & 371.44 \\
\hline
\end{tabular}


Table 10:

Deduced Temperature $\left({ }^{\circ} \mathrm{C}\right)$ vs. Power $(\mathrm{mW})$ data

\begin{tabular}{|c|c|c|c|c|c|}
\hline \multicolumn{2}{|c|}{ Small } & \multicolumn{2}{c|}{ Medium } & \multicolumn{2}{c|}{ Large } \\
\hline $\begin{array}{c}\text { Power } \\
(\mathrm{mW})\end{array}$ & Temp. $\left({ }^{\circ} \mathrm{C}\right)$ & $\begin{array}{c}\text { Power } \\
(\mathrm{mW})\end{array}$ & Temp. $\left({ }^{\circ} \mathrm{C}\right)$ & $\begin{array}{c}\text { Power } \\
(\mathrm{mW})\end{array}$ & Temp. $\left({ }^{\circ} \mathrm{C}\right)$ \\
\hline 0 & 25 & 0 & 25 & 0 & 25 \\
\hline 0.4009 & 27.296 & 0.26043 & 26.98 & 0.2742 & 26.7 \\
\hline 1.592 & 34.809 & 1.0355 & 33.587 & 1.0916 & 31.94 \\
\hline 3.5416 & 46.8 & 2.3083 & 44.055 & 2.438 & 40.04 \\
\hline 6.1974 & 63.731 & 4.0503 & 58.911 & 4.2887 & 51.69 \\
\hline 9.4857 & 86.198 & 6.2204 & 78.77 & 6.6084 & 67.26 \\
\hline 13.3366 & 112.836 & 8.7814 & 102.053 & 9.3648 & 85.45 \\
\hline 17.6581 & 144.403 & 11.6826 & 129.422 & 12.5132 & 106.77 \\
\hline 22.3628 & 180.722 & 14.8697 & 161.151 & 16.0056 & 131.38 \\
\hline 27.3882 & 220.635 & 18.3045 & 196.144 & 19.8039 & 158.61 \\
\hline 32.6549 & 264.422 & 21.9405 & 234.481 & 23.8588 & 188.72 \\
\hline 38.1032 & 311.717 & 25.7415 & 275.756 & 28.1351 & 221.25 \\
\hline 43.6901 & 361.978 & 29.6765 & 319.615 & 32.6035 & 255.77 \\
\hline 49.3591 & 415.408 & 33.7097 & 366.17 & 37.2219 & 292.55 \\
\hline 55.0775 & 471.673 & 37.822 & 414.947 & 41.9693 & 331.15 \\
\hline 60.819 & 530.505 & 41.9954 & 465.691 & 46.8212 & 371.44 \\
\hline 0 & & & & \\
\hline
\end{tabular}




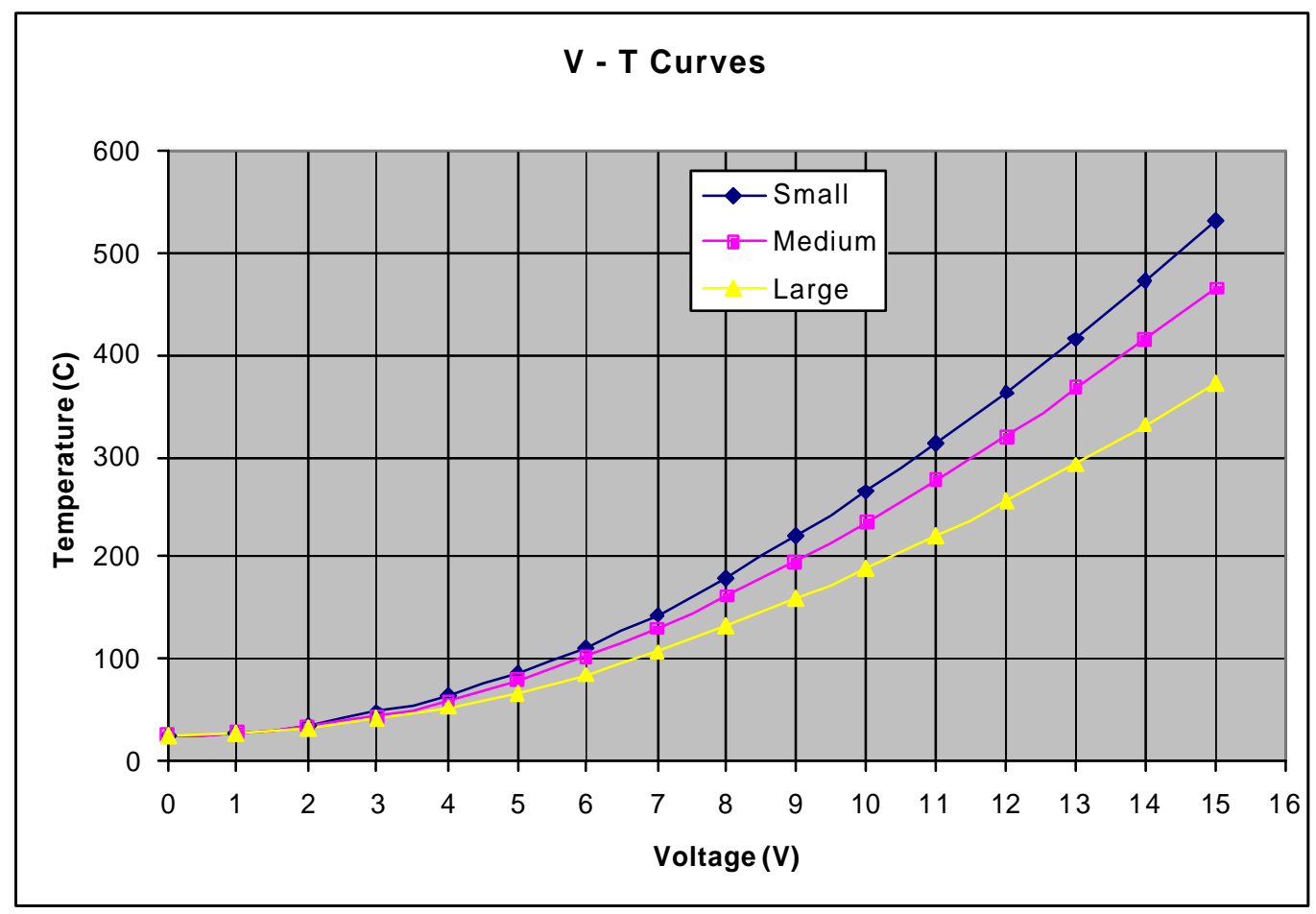

Figure 22: Deduced Temperature $\left({ }^{\circ} \mathrm{C}\right)$ vs. Voltage $(\mathrm{V})$ curves.

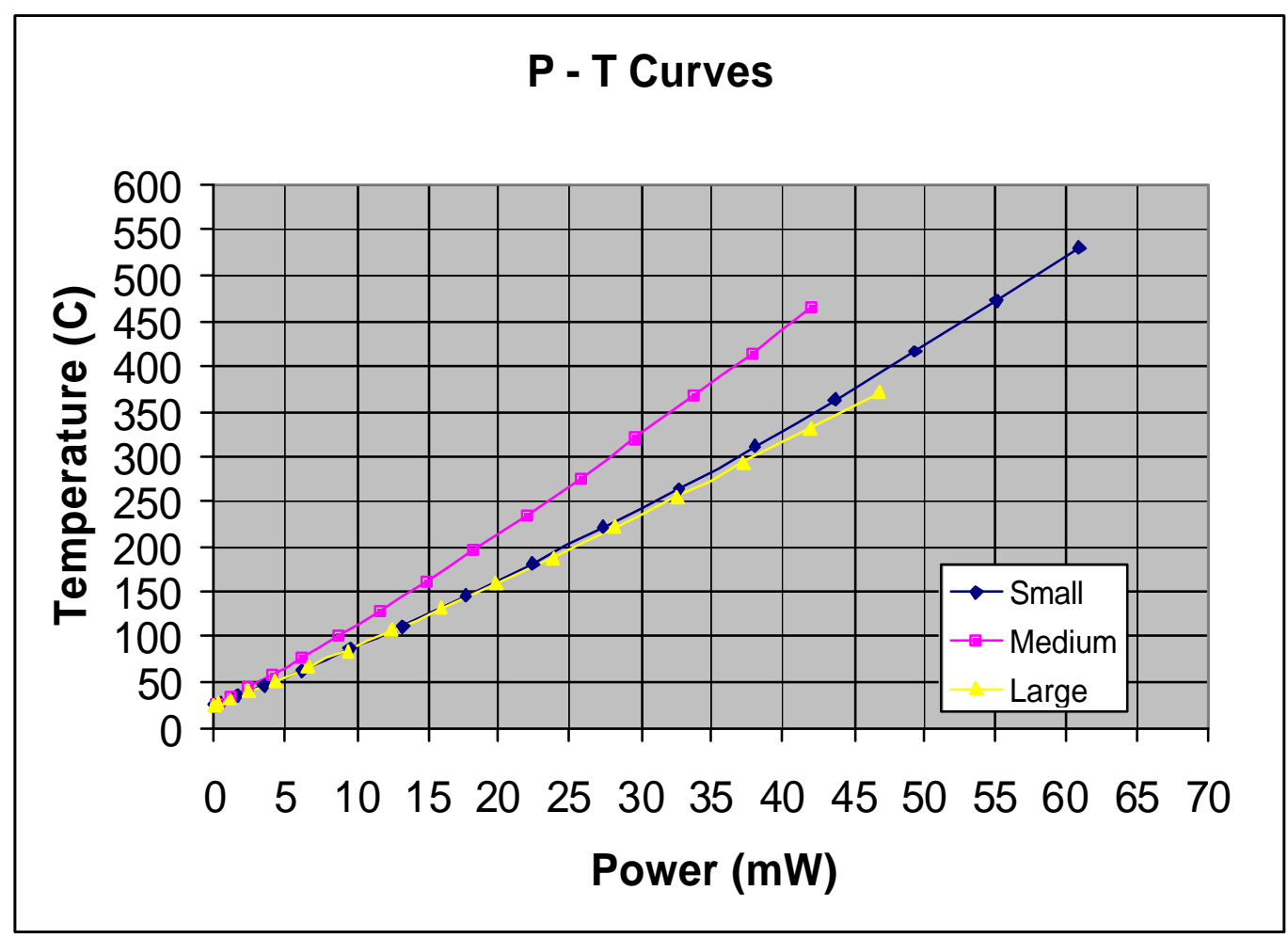

Figure 23: Deduced Temperature $\left({ }^{\circ} \mathrm{C}\right)$ vs. Power $(\mathrm{mW})$ curves. 


\subsection{Material Properties:}

An important consideration for the software is the material properties of the materials used in fabrication. A software cannot distinguish between materials; it can only solve equations based on the mathematical values given to it. CoventorWare has a database known as the Material Properties Database (MPD). The MPD editor has standard materials used in the MEMS field and also allows users to enter custom materials. The values for any material in the database can be varied, changed and stored as per user requirements. A couple of important properties required by the Electro-Thermal solver 'MemETherm' are thermal conductivity and electrical conductivity.

\subsubsection{Electrical Conductivity:}

The electrical conductivity of polysilicon is very important since the voltage is applied to the polysilicon heater. The software uses the applied voltage values and the electrical conductivity to calculate other parameters required for the solution.

Electrical Conductivity of polysilicon is determined by using the experimental results. The resistance of a length of conductor can be defined as [18]:

$$
R=(\rho L / A)
$$

- $A$ can be written as $t^{*} W$

$$
\therefore R=(\rho L / t W)
$$


where: $\rho=$ electrical resistivity $(\mathrm{O} \cdot \mathrm{cm})$

$L=$ length of conductor $(\mathrm{cm})$

$A=$ cross-sectional area $(\mathrm{cm})$

$t=$ thickness $(\mathrm{cm})$

$W=$ width $(\mathrm{cm})$

Electrical Conductivity $\sigma$ is the inverse of electrical resistivity $\rho$ hence;

$$
1 / \rho=(1 / R t)(L / W)
$$

Now, the number of squares $=(\#$ of sqs. $)=(L / W)$

$\therefore$ electrical conductivity $=\sigma=$ (\# of sqs.) / Rt

Using the above equation and the heater dimensions the electrical conductivity of polysilicon is calculated. To determine the number of squares in the heater structure, the following procedure is employed;

The heater is divided into 4 distinct sections that contribute to the number of squares; 'straight', 'diagonal', 'corner' and 'side' as shown in Figure 24. Figures $24,25 \& 26$ show small, medium and large heaters respectively. The dimensions for each of the contributing elements are measured and the number of squares for one element is calculated. Then the total elements for each kind are multiplied and added together to obtain the final value of number of squares for the heater.

The small heater is the only one of the three that has 7 elements (straight), while the medium and large have 9 each (Figures 24, $25 \& 26$ ). Thus the number of squares is less for the small heater. 
Also, the diagonal arms are much thicker with respect to the length which results in a lower value of squares (82.86 squares). This is the same with the large; however the medium heater has a higher ratio in the diagonal arms and hence a higher number of squares. This results in a value of 129.19 squares for the medium heater in comparison with 124.24 squares for the large heater. This is also validated by the data in Table 8 where it is seen that there is a big difference in the resistance of the small and large sizes and only a small difference between the medium and large heaters.

Errors in the calculations may result due to the fact that there are at least four points where the data is approximated; at the two end points and at the junctions of 'side' and 'diagonal'. These have been approximated as straight while the actual value might be less since there is a corner element at the junction.

Using the values for the number of squares and the measured resistance, the electrical conductivity is calculated. The values (of number of squares) and data from Table 8 are used in Equation 11 to obtain the results. The results for all three sizes are tabulated in Table 11 and graphed curves are shown in Figure27.

The data and the curves show that three different sets of values have been obtained for the three sizes. This is unusual since electrical conductivity is a material property and doesn't depend on geometry. The obtained values are evenly spaced and hence the average values obtained for the three are close to the values for the medium hotplate. The disparity in the values could have resulted from the errors in the calculation of the number of squares. 


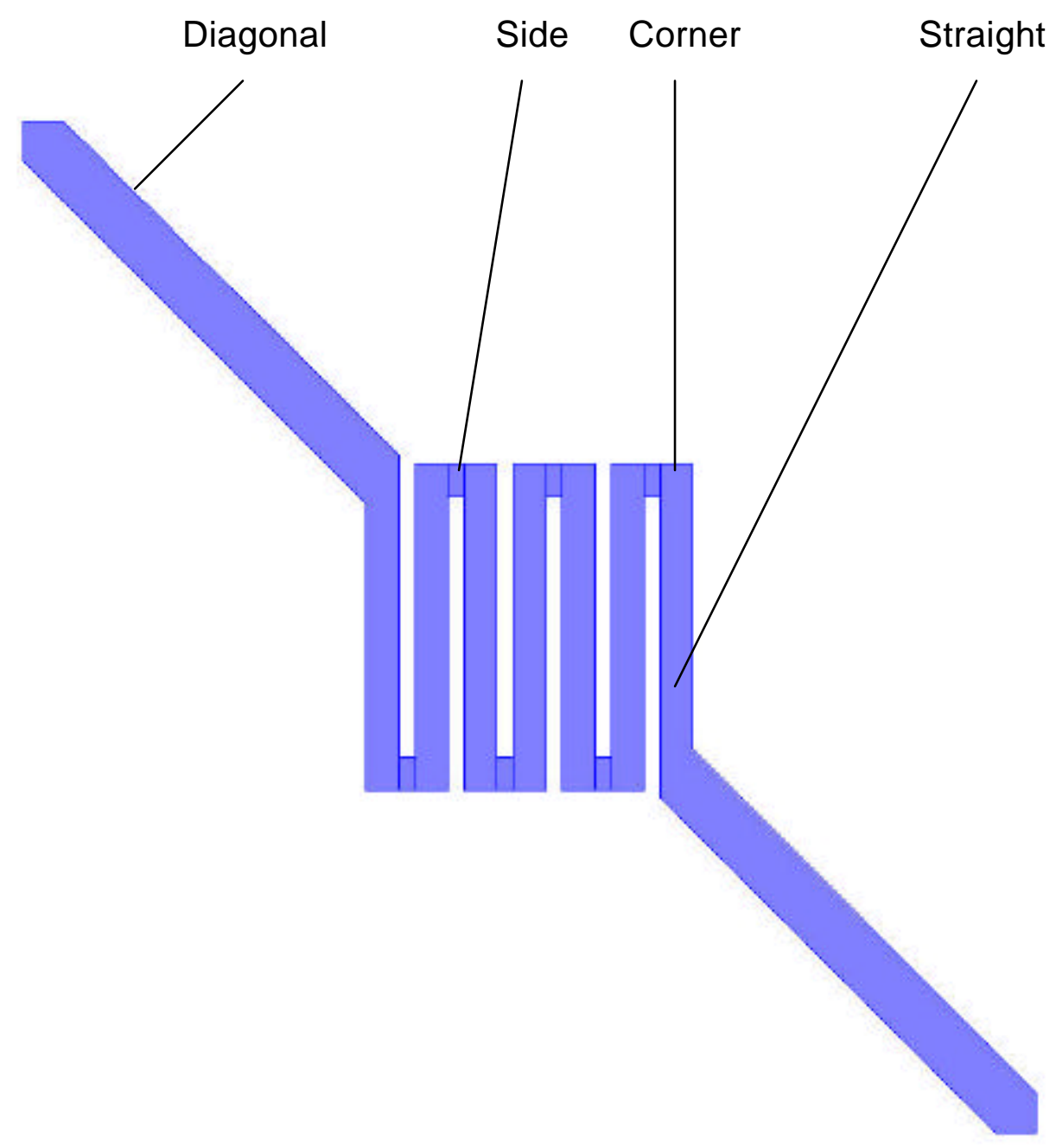

Figure 24: Calculation of number of squares for 'small' heater.

Straight $(64 \mu \mathrm{m}$ by $8 \mu \mathrm{m}) \therefore(\mathrm{L} / \mathrm{W})=64 / 8=8$ sqs.

Diagonal $(120 \mu \mathrm{m}$ by $14 \mu \mathrm{m}) \therefore(\mathrm{L} / \mathrm{W})=120 / 14=8.57$ sqs.

Corners $=0.56$ sqs .

Sides $(4 \mu \mathrm{m}$ by $8 \mu \mathrm{m})=0.5$ sqs.

7 straight +2 diagonal +12 corners +6 sides $=82.86$ sqs . 


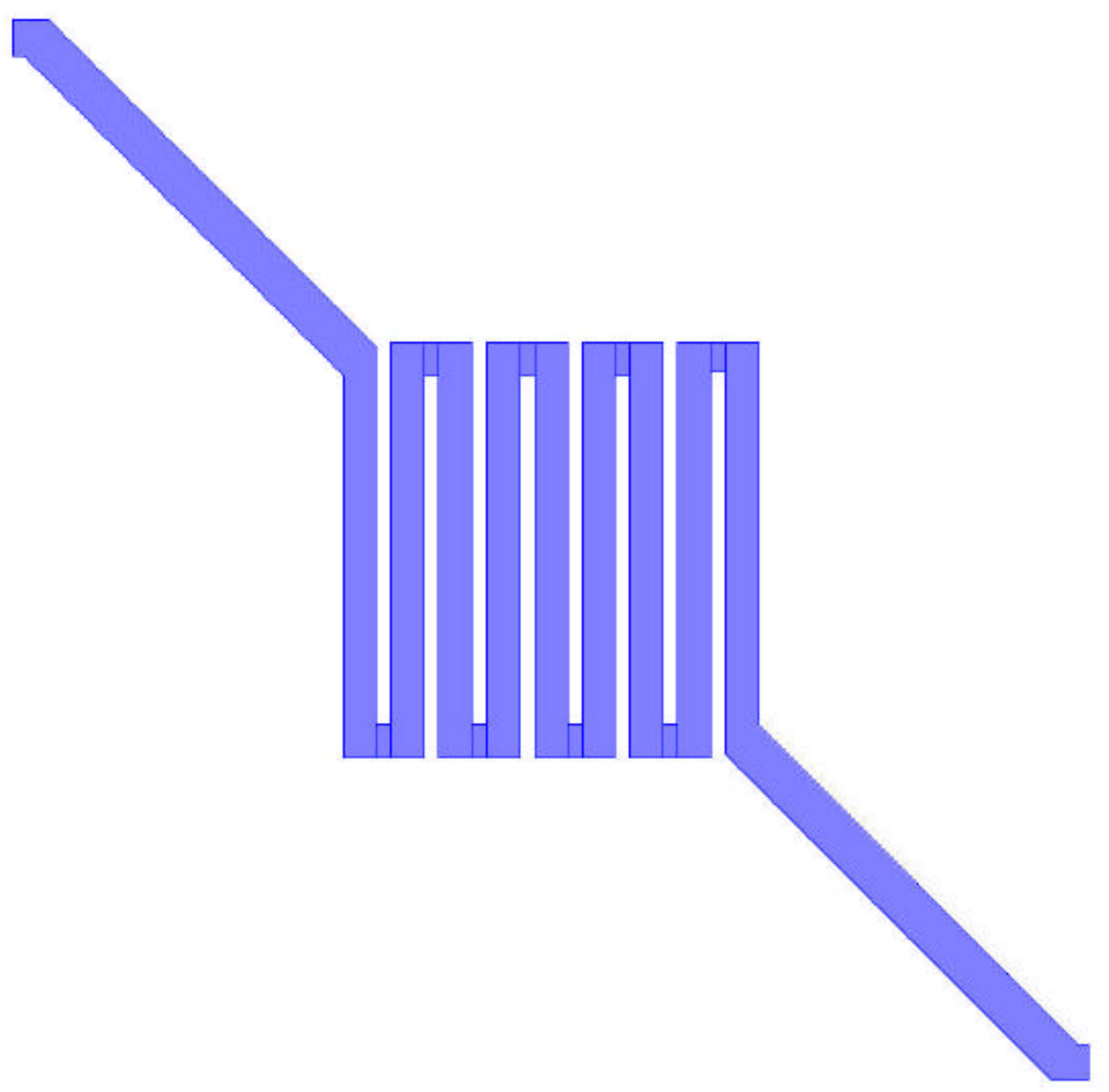

Figure 25: Calculation of number of squares for 'medium' heater.

Straight $(95 \mu \mathrm{m}$ by $9 \mu \mathrm{m}) \therefore(\mathrm{L} / \mathrm{W})=95 / 9=10.56$ sqs.

Diagonal $(130 \mu \mathrm{m}$ by $12 \mu \mathrm{m}) \therefore(\mathrm{L} / \mathrm{W})=130 / 12=10.83$ sqs.

Corners $=0.56$ sqs .

Sides $(4 \mu \mathrm{m}$ by $9 \mu \mathrm{m})=0.44$ sqs.

9 straight +2 diagonal +16 corners +8 sides $=129.19$ sqs . 


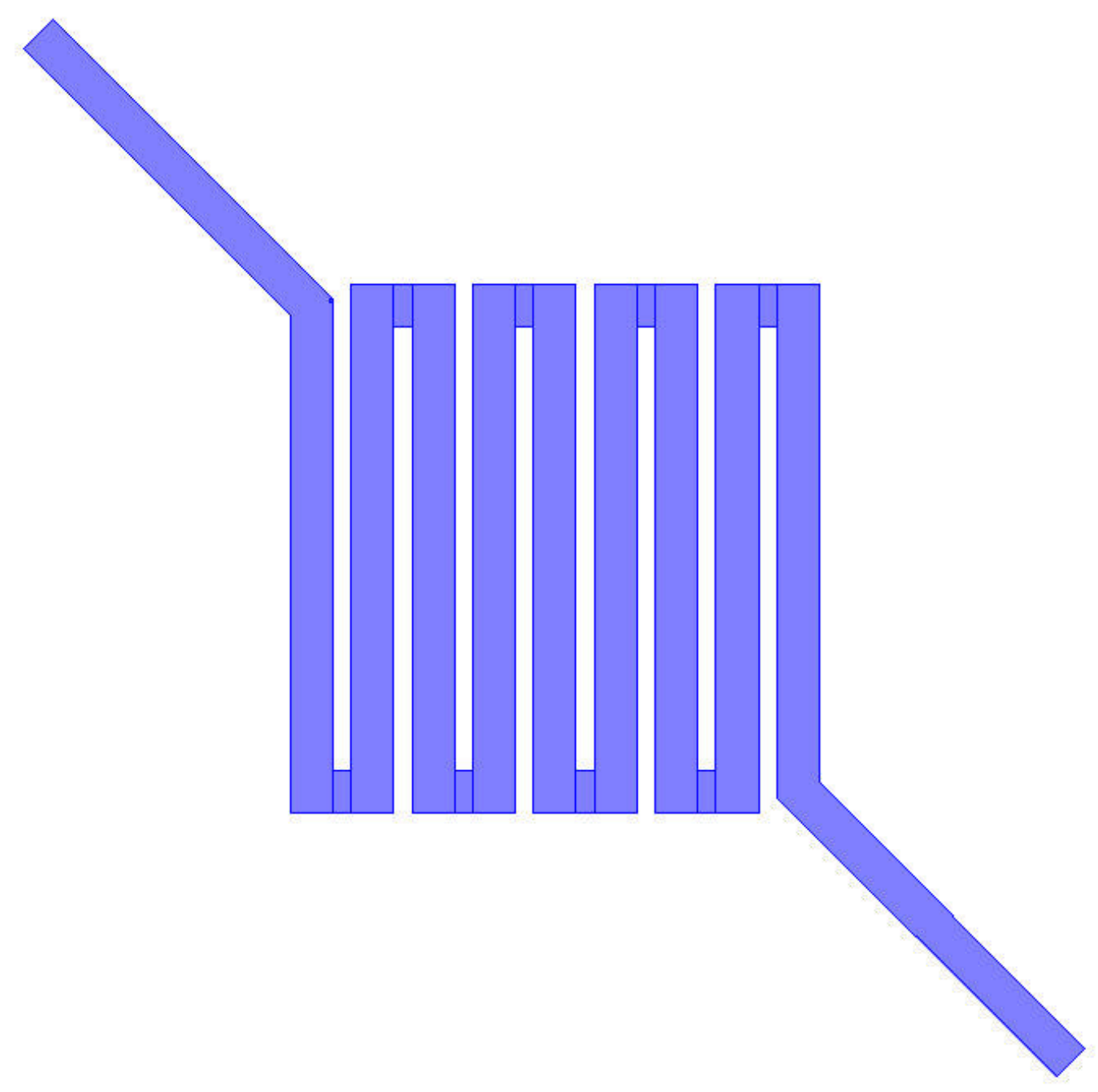

Figure 26: Calculation of number of squares for 'large' heater.

Straight $(146 \mu \mathrm{m}$ by $14 \mu \mathrm{m}) \therefore(\mathrm{L} / \mathrm{W})=146 / 14=10.42$ sqs.

Diagonal $(117 \mu \mathrm{m}$ by $13 \mu \mathrm{m}) \therefore(\mathrm{L} / \mathrm{W})=117 / 13=9$ sqs.

Corners $=0.56$ sqs .

Sides $(6 \mu \mathrm{m}$ by $14 \mu \mathrm{m})=0.43$ sqs.

9 straight +2 diagonal +16 corners +8 sides $=124.24$ sqs . 
Table 11:

Electrical Conductivity of polysilicon heaters.

\begin{tabular}{|c|c|c|c|c|}
\hline \multirow{2}{*}{$\begin{array}{c}\text { Temperature } \\
\left({ }^{\circ} \mathrm{C}\right)\end{array}$} & \multicolumn{4}{|c|}{ Electrical Conductivity (S / m) } \\
\hline & Small & Medium & Large & Average \\
\hline 25 & $8.3226 \mathrm{e}+04$ & $8.4261 \mathrm{e}+04$ & $8.5306 e+04$ & $8.4264 \mathrm{e}+04$ \\
\hline 30 & $8.2860 e+04$ & $8.3900 e+04$ & $8.4939 e+04$ & $8.3900 e+04$ \\
\hline 40 & 8.2120 e+04 & $8.3176 e+04$ & $8.4196 e+04$ & $8.3164 e+04$ \\
\hline 50 & $8.1378 e+04$ & $8.2454 \mathrm{e}+04$ & $8.3443 e+04$ & $8.2425 e+04$ \\
\hline 60 & $8.0640 e+04$ & $8.1736 e+04$ & $8.2694 \mathrm{e}+04$ & $8.1690 \mathrm{e}+04$ \\
\hline 70 & $7.9906 \mathrm{e}+04$ & $8.1027 e+04$ & $8.1952 \mathrm{e}+04$ & $8.0962 e+04$ \\
\hline 80 & $7.9176 \mathrm{e}+04$ & $8.0322 \mathrm{e}+04$ & $8.1223 e+04$ & $8.0240 e+04$ \\
\hline 90 & $7.8454 \mathrm{e}+04$ & 7.9628 e+04 & 8.0508 e+04 & 7.9530 e+04 \\
\hline 100 & $7.7741 \mathrm{e}+04$ & $7.8947 \mathrm{e}+04$ & $7.9804 \mathrm{e}+04$ & $7.8831 \mathrm{e}+04$ \\
\hline 110 & $7.7036 \mathrm{e}+04$ & $7.8277 \mathrm{e}+04$ & $7.9113 \mathrm{e}+04$ & $7.8142 \mathrm{e}+04$ \\
\hline 120 & 7.6340 e+04 & $7.7619 e+04$ & $7.8434 \mathrm{e}+04$ & $7.7464 \mathrm{e}+04$ \\
\hline 130 & $7.5651 \mathrm{e}+04$ & $7.6972 \mathrm{e}+04$ & $7.7766 \mathrm{e}+04$ & 7.6796 e+04 \\
\hline 140 & $7.4972 \mathrm{e}+04$ & $7.6335 \mathrm{e}+04$ & $7.7110 \mathrm{e}+04$ & $7.6139 e+04$ \\
\hline 150 & $7.4300 \mathrm{e}+04$ & $7.5709 \mathrm{e}+04$ & $7.6464 \mathrm{e}+04$ & $7.5491 \mathrm{e}+04$ \\
\hline 160 & 7.3640 e+04 & $7.5093 e+04$ & $7.5830 \mathrm{e}+04$ & $7.4854 \mathrm{e}+04$ \\
\hline 170 & $7.2991 \mathrm{e}+04$ & 7.4486 e+04 & $7.5211 \mathrm{e}+04$ & 7.4229 e+04 \\
\hline 180 & $7.2354 \mathrm{e}+04$ & 7.3898 e+04 & $7.4609 \mathrm{e}+04$ & $7.3620 e+04$ \\
\hline 190 & $7.1727 \mathrm{e}+04$ & $7.3320 \mathrm{e}+04$ & $7.4022 \mathrm{e}+04$ & $7.3023 e+04$ \\
\hline 200 & $7.1112 \mathrm{e}+04$ & $7.2758 e+04$ & $7.3445 e+04$ & $7.2438 \mathrm{e}+04$ \\
\hline
\end{tabular}




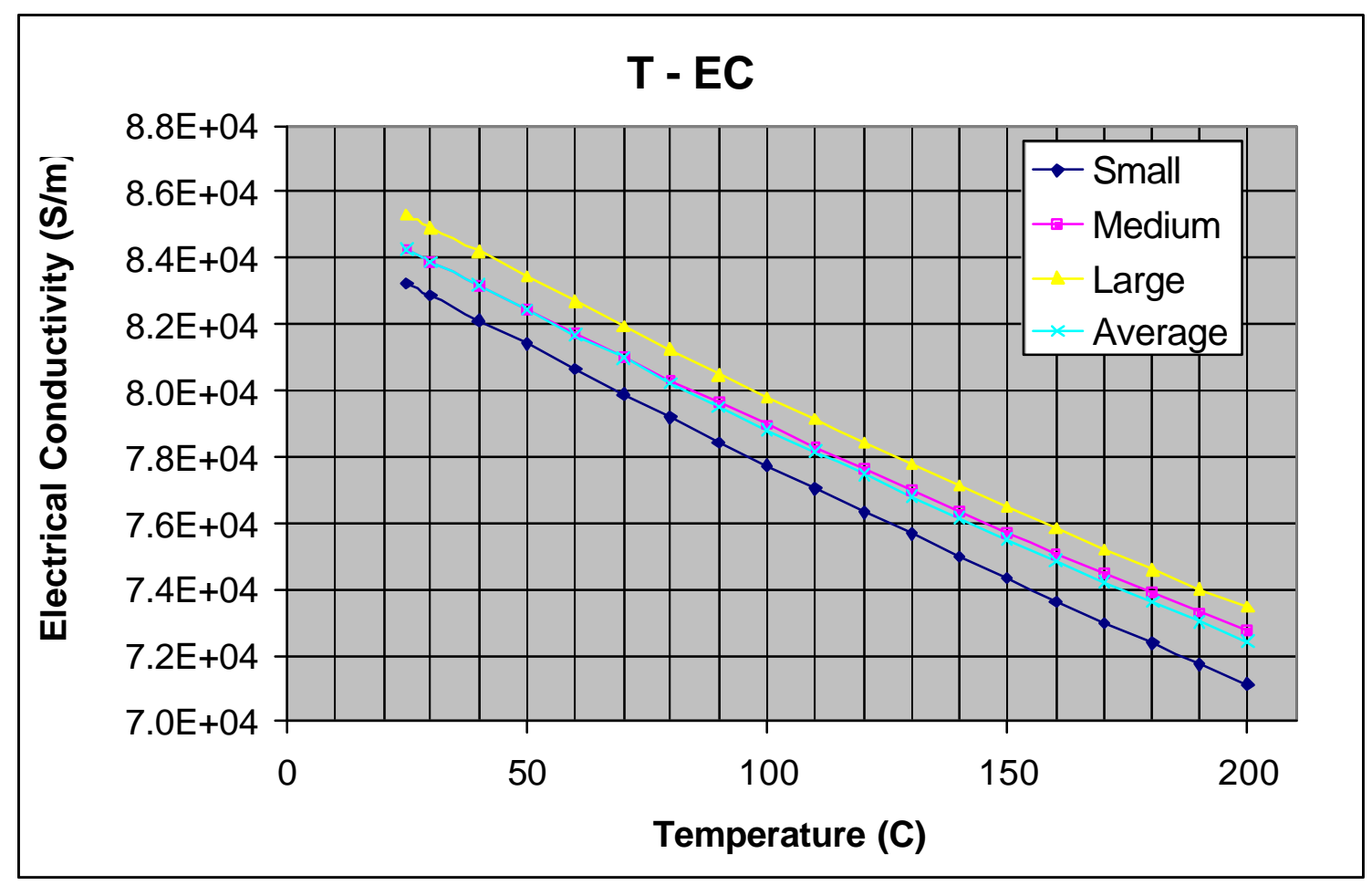

Figure 27: Electrical Conductivity of polysilicon.

From the tabulated values of electrical conductivity of polysilicon, only 10 values are used since Coventorware allows a maximum of 10 entries in its MPD editor table. Since the software works on devices at the micron scale, the acceptable unit for electrical conductivity is $\mathrm{pS} / \mu \mathrm{m}$. Hence the values have to be converted to these units before storing in CoventorWare. The values are tabulated in Table 12.

Apart from the electrical conductivity of polysilicon, other properties (at $300 \mathrm{~K}$ ) are:

1) Thermal Conductivity of polysilicon $=13.6 \mathrm{~W} / \mathrm{m} \cdot \mathrm{K}[19]$

2) Thermal Conductivity of oxide $=1.4226 \mathrm{~W} / \mathrm{m} \cdot \mathrm{K}$ [9]

3) Electrical Conductivity of aluminum $=1.82 \mathrm{e}+07 \mathrm{~S} / \mathrm{m}[20]$

4) Thermal Conductivity of aluminum: (see Table 11) [1, 20] 
Table 12: Electrical conductivity values for CoventorWare

\begin{tabular}{|c|c|}
\hline Temp. $\left({ }^{\circ} \mathrm{C}\right)$ & EC $(\mathrm{S} / \mathrm{m})$ \\
\hline 27 & $8.4264 \mathrm{e}+04$ \\
\hline 40 & $8.3164 \mathrm{e}+04$ \\
\hline 50 & $8.2425 \mathrm{e}+04$ \\
\hline 70 & $8.0962 \mathrm{e}+04$ \\
\hline 100 & $7.8831 \mathrm{e}+04$ \\
\hline 120 & $7.7464 \mathrm{e}+04$ \\
\hline 140 & $7.6139 \mathrm{e}+04$ \\
\hline 160 & $7.4854 \mathrm{e}+04$ \\
\hline 180 & $7.3620 \mathrm{e}+04$ \\
\hline 200 & $7.2438 \mathrm{e}+04$ \\
\hline
\end{tabular}

Table 13: Thermal Conductivity of aluminum $[1,20]$

\begin{tabular}{|c|c|}
\hline $\begin{array}{c}\text { Temp. } \\
(\mathrm{K})\end{array}$ & $\begin{array}{c}\text { TC } \\
(\mathrm{W} / \mathrm{m} \cdot \mathrm{K})\end{array}$ \\
\hline 100 & 302 \\
\hline 200 & 237 \\
\hline 300 & 237 \\
\hline 400 & 240 \\
\hline 600 & 231 \\
\hline 800 & 218 \\
\hline
\end{tabular}

The values in Table 13 are taken from two different sources with six data points available within the range of the hotplate temperatures. Again, these values have to be converted into an acceptable scientific notation $(\mathrm{pW} / \mu \mathrm{m} \cdot \mathrm{K})$ for CoventorWare. 


\subsection{Solver Set-up}

Once the software builds the solid model and the mesh from the designs, the next step is to isolate and identify the surfaces where the inputs are applied and outputs are measured. This is done in the mesh window itself where the surfaces are called "patches" and each patch can be assigned a unique name. These patches can then be used to apply different boundary conditions (inputs) in the solver set-up window.

For the heater, the patches are named as 'Vin' and 'Vout' as shown in Figure 28. The patch 'Vin' is the vertical surface of the heater (the ' $z$ ' direction is exaggerated). Similarly, 'Vout' (not visible) is the surface at the opposite end.

Other patches used in the substrate are specified in Figure 29. The picture shows an inverted view of the substrate with patches 'bottom', 'botox' and 'side'.

Since Figure 29 shows an inverted device, the top surface is not visible. The entire top surface of the device is denoted as the patch 'top'. 'botox' is applied only to the bottom layer of oxide 0 while the bottom of the substrate is named as 'bottom'. The patch 'side' is made up of 24 different patches. It includes all 4 sides of the square for all layers of the device; i.e. 6 layers and 4 sides each.

Once the patches have been defined, the solver is set up by applying various boundary conditions. For a simple single voltage simulation, the ambient (starting) temperature, convection coefficients and the voltage are assigned to the respective surfaces. 


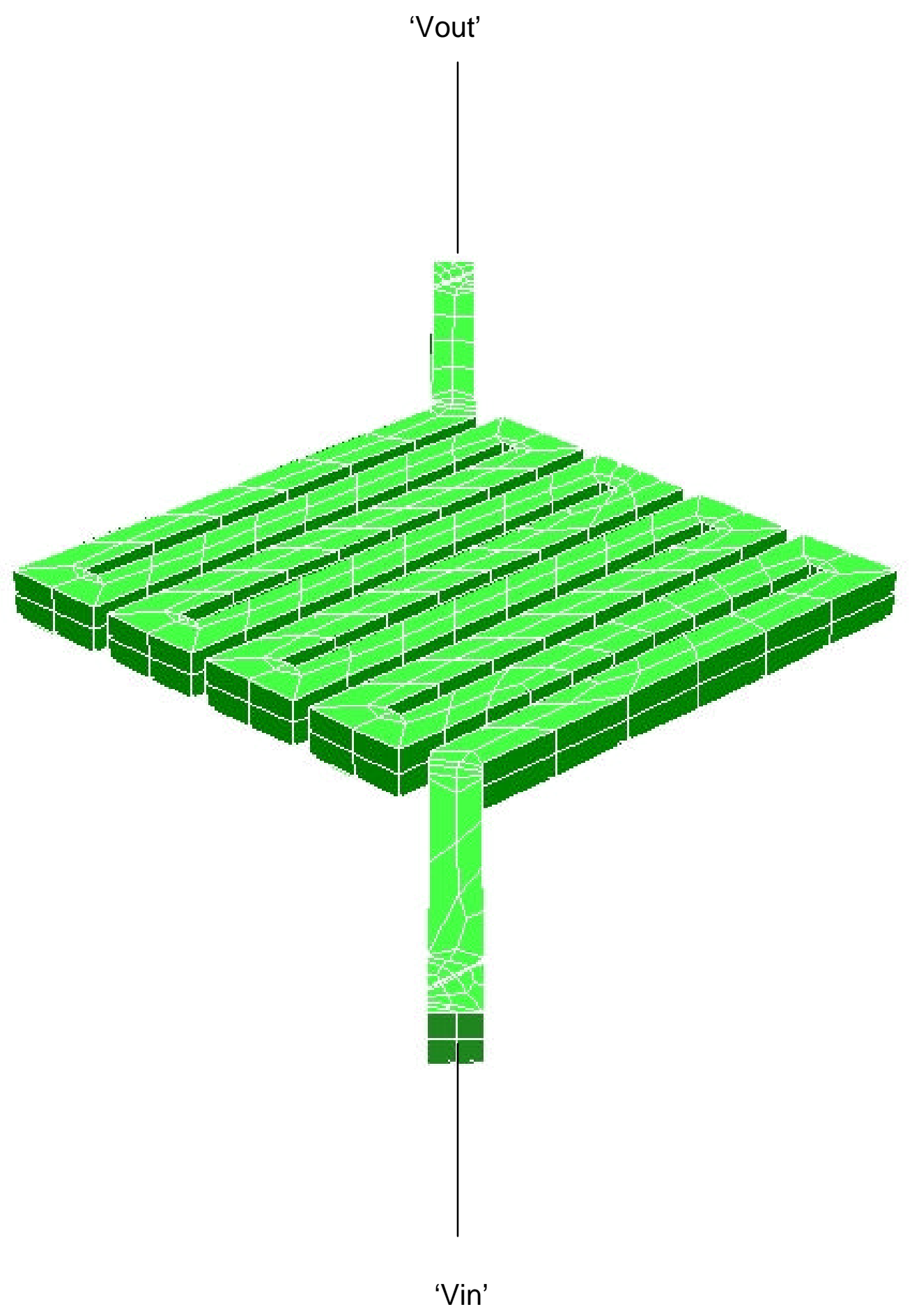

Figure 28: Heater patch naming. 


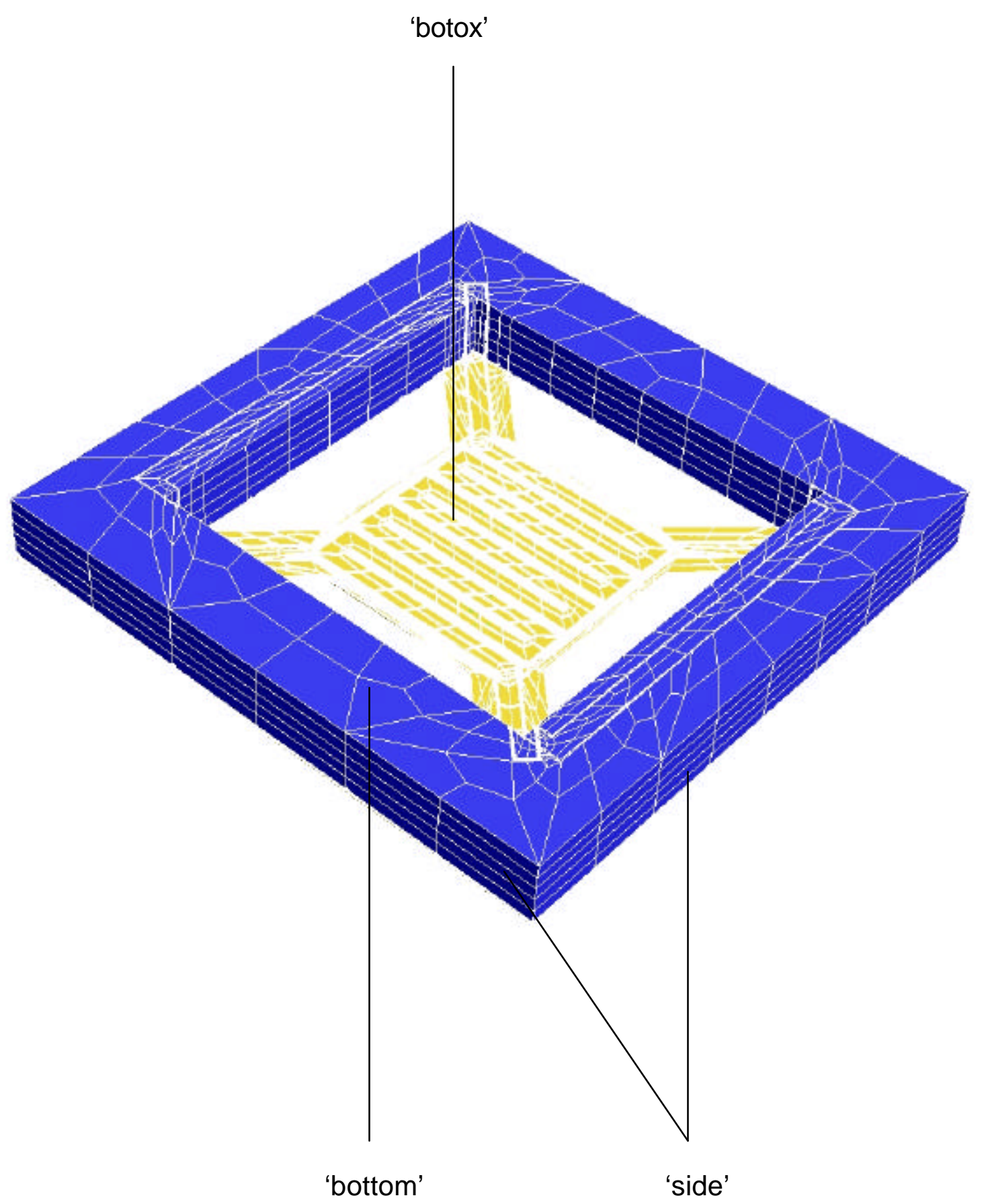

Figure 29: Substrate patch naming. 
As shown in Figure 30, a temperature of $300 \mathrm{~K}$ is applied to the patches 'Vin', 'Vout' and 'side'. 'Vin' is applied a voltage of $1 \mathrm{~V}$ while 'Vout' is $0 \mathrm{~V}$. The value of 'Vin' can be changed as per the simulation requirements. Convection boundary conditions are set up in a separate window which opens up when "ConvectionRadiation" is selected (see Figure 31).

\begin{tabular}{|c|c|c|c|c|c|c|c|c|c|}
\hline syroutcls & Fxilye & Pattol & and & Pittl? & and? & Pattos & \multicolumn{2}{|c|}{ Losidolde } & lialible \\
\hline Sitt & Temorature & Difin & $200^{\circ}$ & rod & 0 & side & Sorater & 300 & Finet \\
\hline Stet & nowe & ] nome & and & ] none & 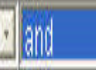 & mone & Sicalar & 0 & Fived \\
\hline Seth & Nillane & ] liin & and & anene & 1. 20011 & rome & Shatal & 1.) & ET BC: \\
\hline Set4 & Winlane & 2 roul & 2. and & ] nowe & 2 and & rowe & Solat & 0.1. & Fined \\
\hline $50 t^{5}$ & Comedion:Radition & $\sqrt{1000}$ & and & mine & 2. & mone & 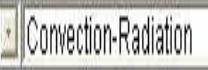 & Ed: & Fined \\
\hline $5066^{\circ}$ & Comeditor-Ralition & . & and & n & and & rowe & 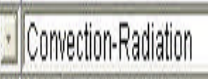 & Ed: & Finged \\
\hline 5et? & nones & mone & and & nine & 2) 2 and & Mone & Fialai & 0 & Fined \\
\hline 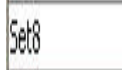 & none & nowe & 2and & mone & - & nome & Piolat & 0.0 & Fined \\
\hline
\end{tabular}

Figure 30: Boundary conditions for solver set up.

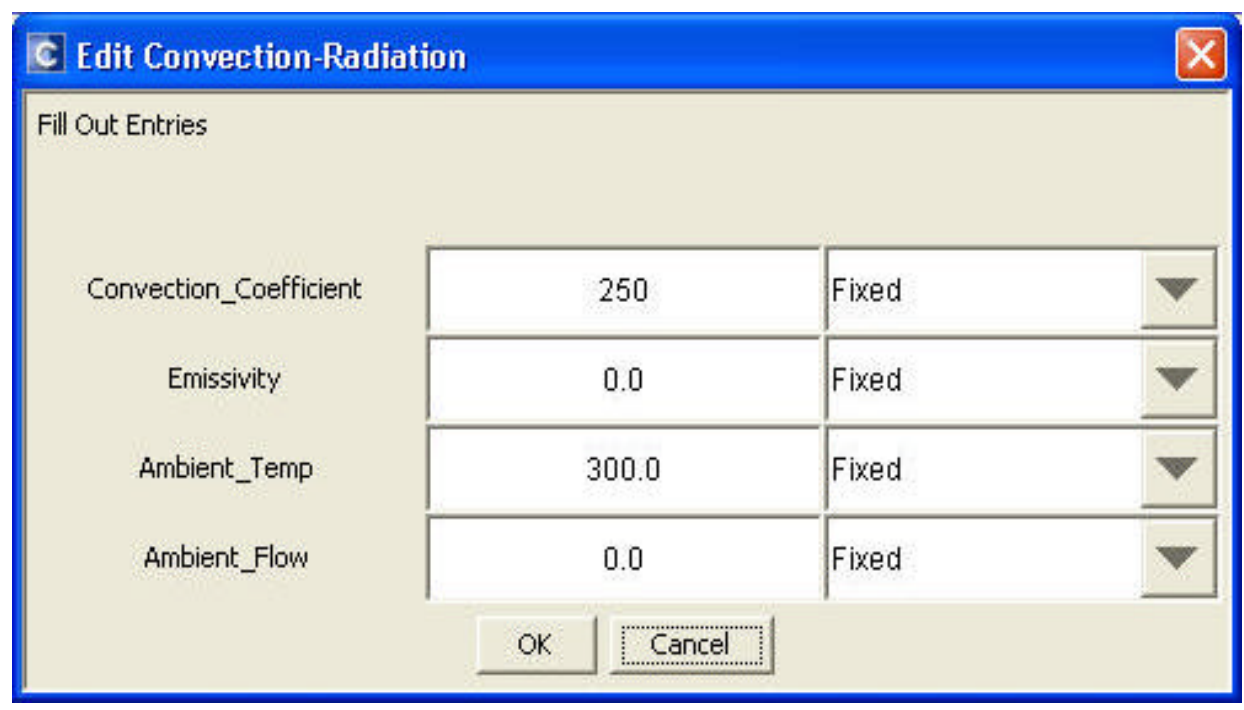

Figure 31: Setting convection boundary conditions. 
Ambient temperature is set at $300 \mathrm{~K}$ while the actual convection coefficient is changed for different simulations.

In Figure 30, the patch 'bottom' is not assigned any convection values because for the experimental setup the chip substrate is in direct contact with the thermal chuck and as such has no airflow.

Once the solver has run a single input simulation, it is prepared for a parametric (varying input) simulation. In the main window (Figure 30) the input 'Vin' is associated with a variable 'ET_BC1'. This variable is associated with a trajectory 't1' in a separate window (Figure 32). This allows the input variable 'Vin' to be varied according to the trajectory assigned to 't1'. Examples of trajectories are step, delta, logarithmic etc. The trajectory chosen for 't1' is 'delta' which varies from $3 \mathrm{~V}$ to $15 \mathrm{~V}$ in steps of $3 \mathrm{~V}$ each (Figure 33).

\begin{tabular}{|c|c|c|c|c|c|c|}
\hline \multicolumn{6}{|l|}{ C electhermBCs } & $\mathrm{X}$ \\
\hline electhermBCs & \multicolumn{2}{|c|}{ Trajectory } & \multicolumn{2}{|c|}{ Type } & ScaleFactor & \\
\hline ET_BC1 & t1 & ב & Factor & 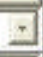 & $1.000000 \mathrm{E} 00$ & \\
\hline ET_BC2 & none & ב] & Factor & $\exists$ & 1.000000E00 & \\
\hline ET_BC3 & none & 국 & Factor & $\sqrt{2}$ & $1.000000 \mathrm{E} 00$ & \\
\hline ET_BC4 & none & $\exists$ & Factor & 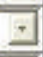 & $1.000000 \mathrm{E} 00$ & \\
\hline ET_BC5 & none & 교 & Factor & $\exists$ & 1.000000E00 & \\
\hline ET_BC6 & none & $\exists$ & Factor & $\sqrt{7}$ & $1.000000 \mathrm{E} 00$ & \\
\hline ET_BC7 & none & 교 & Factor & $\sqrt{2}$ & $1.000000 \mathrm{E} 00$ & \\
\hline \multirow[t]{2}{*}{ ET_BC8 } & none & $\exists$ & Factor & ב & $1.000000 \mathrm{E} 00$ & \\
\hline & & OK & Cancel & & & ?? \\
\hline
\end{tabular}

Figure 32: Electro-Thermal boundary conditions for parametric solution. 


\begin{tabular}{|c|c|c|c|c|c|c|}
\hline Trajectories & \multicolumn{3}{|c|}{ TrajectoryType } & Label & \multirow{3}{*}{ Start: } & \multirow{3}{*}{30} \\
\hline$t 1$ & Delta & 1 & Edit: & voltade & & \\
\hline 2 & None & $\exists$ & - & t2 & & \\
\hline 13 & None & 7 & - & 13 & Delta: & 3.0 \\
\hline & & & & & Stop: & 15.0 \\
\hline & & & & & OK & Cancel \\
\hline
\end{tabular}

Figure 33: Trajectory 't1' with 'delta' variation.

\subsection{Design of Experiments:}

The main focus of this research work is to obtain a suitable value of convection coefficients for the micro-hotplates. To this end, numerous simulations are performed by Coventorware with varying values of convection coefficients. The starting point are the values $125 \mathrm{~W} / \mathrm{m} \cdot \mathrm{K}$ and $60 \mathrm{~W} / \mathrm{m} \cdot \mathrm{K}$ for the top and bottom surfaces respectively. These values have been used in successful experiments for similar hotplates by at least two groups [9, 11]. The values are denoted as the nominal value $125 / 60$, where the first number is the value of $h$ for the top surface and the second is for the bottom surface. Then the convection coefficients are varied from the nominal value by $\pm 20 \%$ as well as changing the ratio of the top to bottom surface. Table 14 shows a better picture of the values. 


\section{Table 14:}

Values of convection coefficients $[9,11]$

\begin{tabular}{|c|c|c|}
\hline$-20 \%$ & $h(\mathrm{~W} / \mathrm{m} \cdot \mathrm{K})$ & $+20 \%$ \\
\hline- & $125 / 90$ & - \\
\hline $100 / 48$ & $125 / 60$ & $150 / 72$ \\
\hline- & $125 / 30$ & - \\
\hline
\end{tabular}

Apart from these values, convection coefficients were calculated using the equation [1];

$$
h=N u_{L} k / L
$$

For upper surface,

$$
N u_{L}=0.54\left(R a_{L}\right)^{1 / 5}
$$

For lower surface,

$$
N u_{L}=0.27\left(R a_{L}\right)^{1 / 5}
$$

and,

$$
R a_{L}=\left[g B\left(T_{s}-T_{T}\right) L^{3}\right] / v a
$$

where: $h=$ convection coefficient $\left(\mathrm{W} / \mathrm{m}^{2} \cdot \mathrm{K}\right)$

$N u_{L}=$ Nusselt number

$R a_{L}=$ Rayleigh number

$g=$ gravitational force $\left(\mathrm{m} / \mathrm{s}^{2}\right)$

$B=$ expansion coefficient $\left(\mathrm{K}^{-1}\right)$

$T_{s}=$ surface temperature $(\mathrm{K})$

$T_{T}=$ ambient temperature $(\mathrm{K})$ 
$L=$ length of side $(\mathrm{m})$

$v=$ velocity component normal to the flow $\left(\mathrm{m}^{2} / \mathrm{s}\right)$

These equations hold true for macro-scale calculations, hence the values obtained from these calculations are only an approximation of the micro-scale values. Values of $h$ are calculated for an ambient temperature of $300 \mathrm{~K}$ and a surface temperature of $400 \mathrm{~K}$ for each of the hotplates. The obtained results are tabulated in Table 15. The nominal values are referenced from previous work which feature similar hotplates but are of different dimensions, while the calculated values are specifically for the hotplates used in this research.

\section{Table 15:}

Calculated values of convection coefficients: $h\left(\mathrm{~W} / \mathrm{m}^{2} \cdot \mathrm{K}\right)$.

\begin{tabular}{|c|c|c|c|}
\hline & Small & Medium & Large \\
\hline Upper & 56 & 50 & 47 \\
\hline Lower & 28 & 25 & 23.5 \\
\hline
\end{tabular}

The first simulation is performed for the above given values and then further values are determined by observing the simulation results for these values. Four sets of convection coefficients are used for each hotplate starting from the values listed in Table 15. 


\section{CHAPTER 4}

\section{RESULTS and DISCUSSION}

The results obtained from the simulations are recorded in Tables 14-19. There are two sets of data; one for each set of convection coefficients. The 3-D simulation results are shown in Figures $35,36 \& 37$. It is clearly seen that the suspended structure is heated uniformly without any major variations over the entire surface. The substrate is at enough distance from the heater that it remains at ambient temperature.

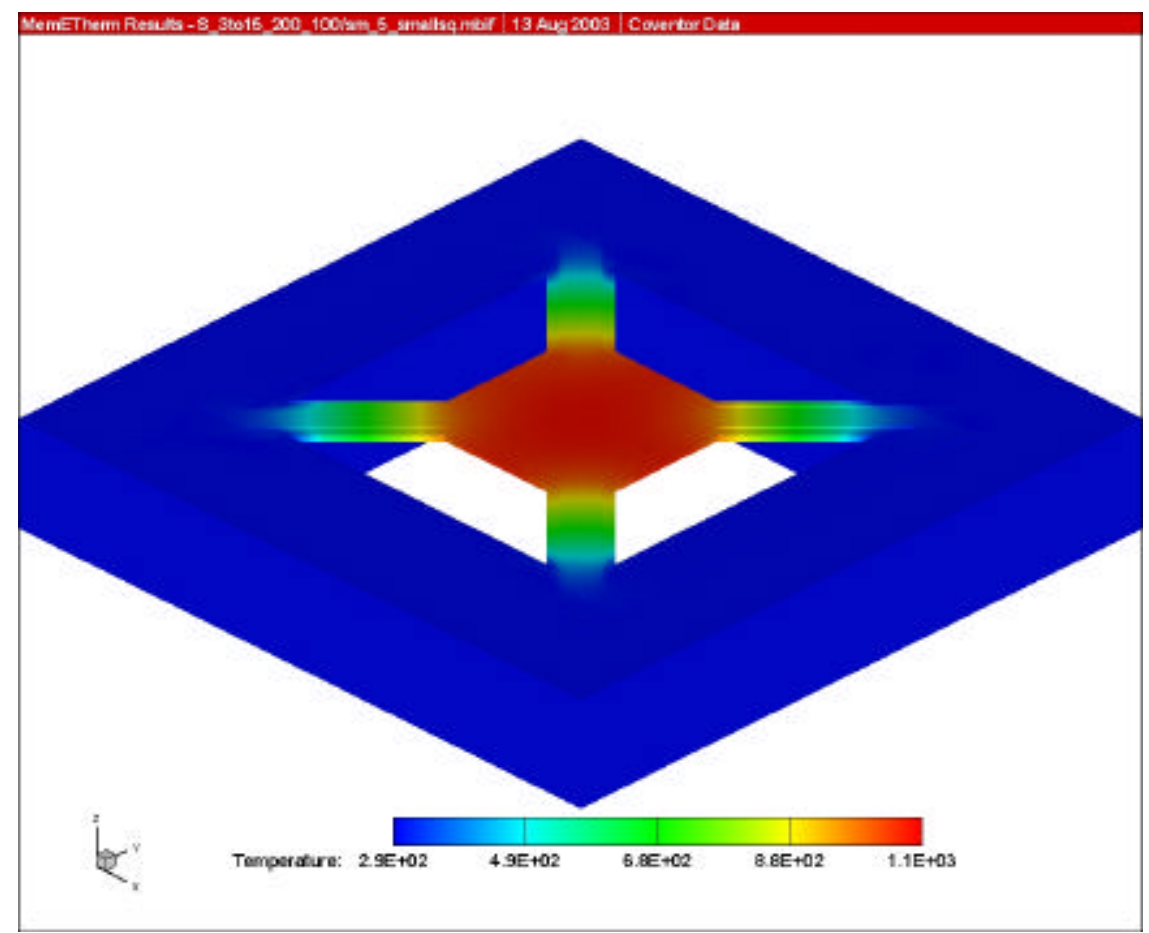

Figure 34: Small hotplate - 3D view $(h=200 / 100)$ 


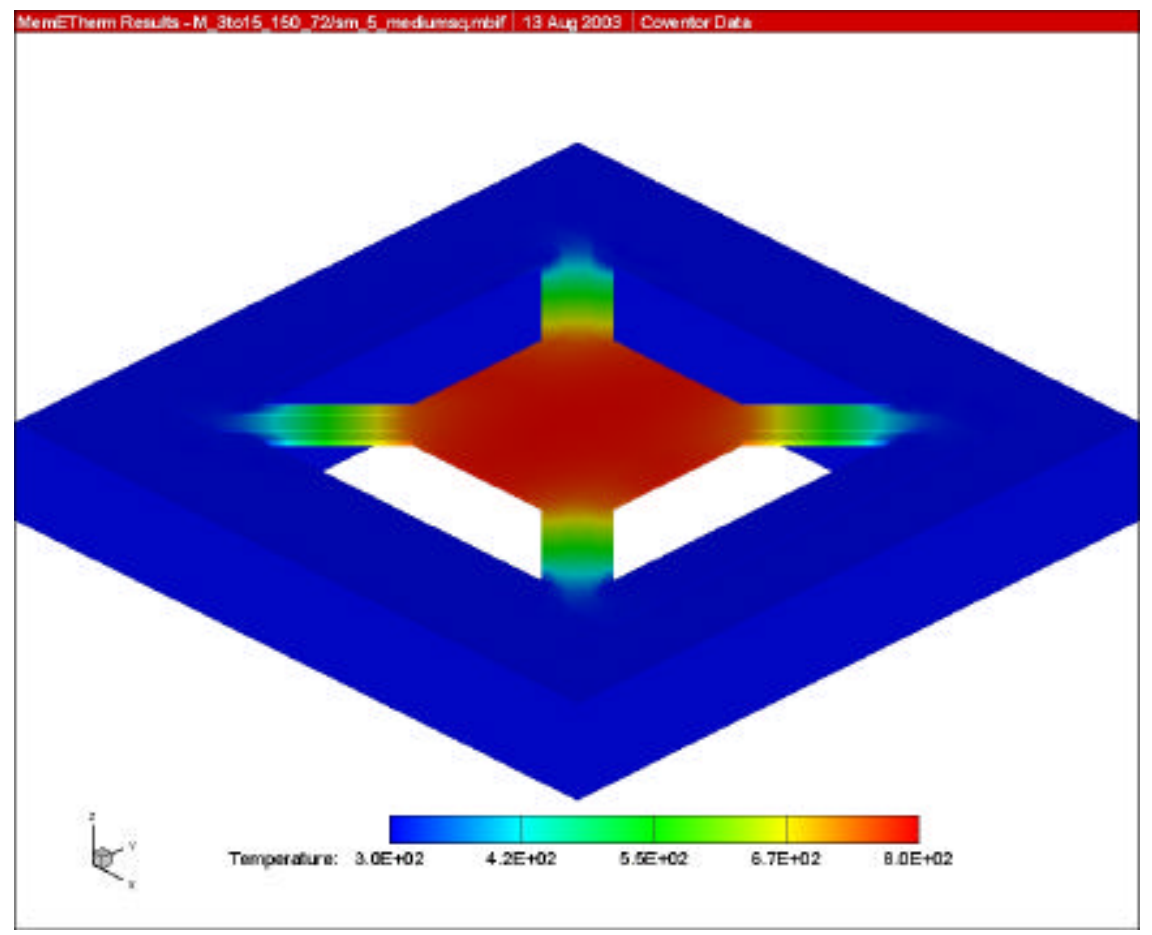

Figure 35: Medium hotplate - 3D view $(h=150 / 72)$

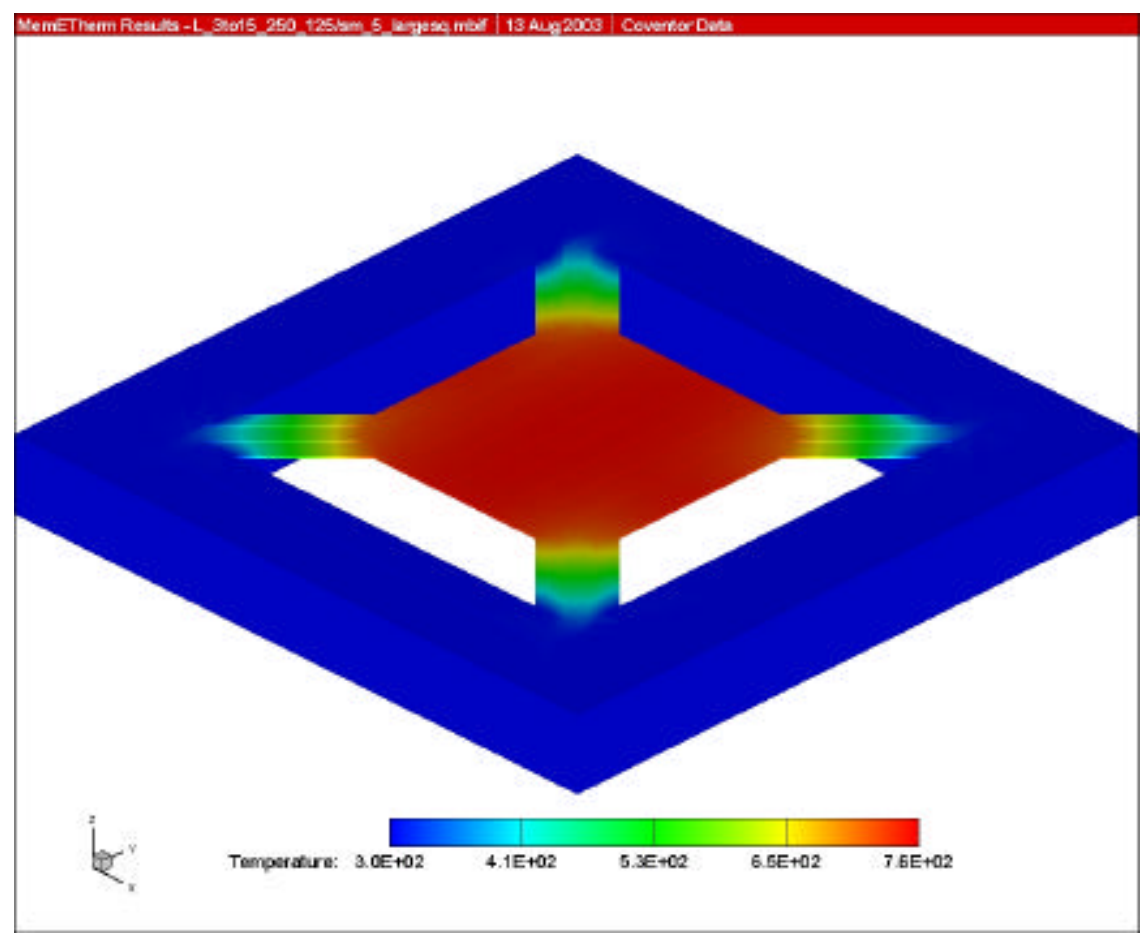

Figure 36: Large hotplate-3D view $(h=250 / 125)$ 
The 3-D pictures from CoventorWare use the same color scheme for all the simulation results. There is no variation in the display apart from the scale displaying the temperature. Hence a result for a $125 / 90$ simulation for a small hotplate would look exactly the same as one for a 200/100 simulation for a large hotplate (Figures 34, 35 and 36).

Tables $14-19$ contain simulation data for the referenced and calculated convection coefficient values while Figures 37,38 and 39 show the error curves.

The error is calculated as;

$$
\% \text { error }=(T S-T E) / T E * 100
$$

where: $\mathrm{TS}=$ Simulated Temperature $\left({ }^{\circ} \mathrm{C}\right)$ $\mathrm{TE}=$ Experimental Temperature $\left({ }^{\circ} \mathrm{C}\right)$

For the small and the medium hotplates, it is noticed that the curves for Tables 14 and 15 form two groups, which are far apart from each other. However, the curves within each group are bunched together (Figures 37 and 38). The curves for the referenced values are quite close together with not much difference between different values of $h$. For the calculated values (see Table 16) the best match is provided by the values $200 / 100$, however the difference between the best $(200 / 100)$ and the poorest (56/28) match ranges from $1.3 \%(3 \mathrm{~V})$ to $2.9 \%$ (15V). Hence convective heat transfer is less for this size of hotplate, supporting the findings of S. Astie et. al. [9]. 
Table 16:

Small hotplate -results

\begin{tabular}{|c|c|c|c|c|c|}
\hline \multirow{2}{*}{$\begin{array}{c}\text { Voltage } \\
(\text { V) }\end{array}$} & \multicolumn{5}{|c|}{ Temperature $\left({ }^{\circ} \mathbf{C}\right)$} \\
\cline { 2 - 6 } & Expt. & $\mathbf{1 2 5 / 9 0}$ & $\mathbf{1 2 5 / 6 0}$ & $\mathbf{1 2 5 / 3 0}$ & $\mathbf{1 0 0 / 4 8}$ \\
\hline 0.00 & 25.00 & 25.00 & 25.00 & 25.00 & 25.00 \\
\hline 3.00 & 46.80 & 47.19 & 47.26 & 47.32 & 47.33 \\
\hline 6.00 & 112.84 & 103.56 & 103.78 & 104.00 & 104.05 \\
\hline 9.00 & 220.64 & 191.19 & 191.69 & 192.19 & 192.30 \\
\hline 12.00 & 361.98 & 317.20 & 318.08 & 318.97 & 319.17 \\
\hline 15.00 & 530.51 & 479.04 & 480.34 & 481.81 & 482.12 \\
\hline- & \multicolumn{5}{|c|}{$\%$ Error } \\
\hline 0.00 & - & 0.00 & 0.00 & 0.00 & 0.00 \\
\hline 3.00 & - & 0.83 & 0.98 & 1.11 & 1.13 \\
\hline 6.00 & - & -8.22 & -8.03 & -7.83 & -7.79 \\
\hline 9.00 & - & -13.35 & -13.12 & -12.89 & -12.84 \\
\hline 12.00 & - & -12.37 & -12.13 & -11.88 & -11.83 \\
\hline 15.00 & - & -9.70 & -9.46 & -9.18 & -9.12 \\
\hline \multirow{6}{|c|}{ Temperature $\left({ }^{\circ} \mathbf{C}\right)$} \\
\cline { 2 - 6 } & $\mathbf{1 5 0 / 7 2}$ & $\mathbf{5 6 / 2 8}$ & $\mathbf{8 4 / 4 2}$ & $\mathbf{1 5 0 / 7 5}$ & $\mathbf{2 0 0 / 1 0 0}$ \\
\hline 0.00 & 25.00 & 25.00 & 25.00 & 25.00 & 25.00 \\
\hline 3.00 & 47.18 & 51.83 & 51.71 & 51.43 & 51.22 \\
\hline 6.00 & 103.51 & 119.96 & 119.53 & 118.54 & 117.80 \\
\hline 9.00 & 191.08 & 229.54 & 228.51 & 226.14 & 224.37 \\
\hline 12.00 & 317.00 & 391.77 & 389.83 & 385.32 & 381.98 \\
\hline 15.00 & 478.74 & 606.86 & 603.74 & 596.52 & 591.18 \\
\hline- & \multicolumn{5}{|c|}{$\%$ Error } \\
\hline 0.00 & 0.00 & 0.00 & 0.00 & 0.00 & 0.00 \\
\hline 3.00 & 0.81 & 10.75 & 10.49 & 9.89 & 9.44 \\
\hline 6.00 & -8.27 & 6.31 & 5.93 & 6.06 & 4.40 \\
\hline 9.00 & -13.40 & 4.04 & 3.57 & 2.50 & 1.69 \\
\hline 12.00 & -12.43 & 8.23 & 7.69 & 6.45 & 5.53 \\
\hline 15.00 & -9.76 & 14.39 & 13.80 & 12.44 & 11.44 \\
\hline
\end{tabular}


Table 17:

Medium hotplate - results

\begin{tabular}{|c|c|c|c|c|c|}
\hline \multirow{2}{*}{$\begin{array}{c}\text { Voltage } \\
(\text { V) }\end{array}$} & \multicolumn{5}{|c|}{ Temperature $\left({ }^{\circ} \mathbf{C}\right)$} \\
\cline { 2 - 6 } & Expt. & $\mathbf{1 2 5 / 9 0}$ & $\mathbf{1 2 5 / 6 0}$ & $\mathbf{1 2 5 / 3 0}$ & $\mathbf{1 0 0 / 4 8}$ \\
\hline 0.00 & 25.00 & 25.00 & 25.00 & 25.00 & 25.00 \\
\hline 3.00 & 44.06 & 42.98 & 43.08 & 43.18 & 43.20 \\
\hline 6.00 & 102.05 & 88.08 & 88.44 & 88.81 & 88.89 \\
\hline 9.00 & 196.14 & 156.14 & 156.92 & 157.72 & 157.90 \\
\hline 12.00 & 319.62 & 255.22 & 256.63 & 258.06 & 258.39 \\
\hline 15.00 & 465.69 & 382.53 & 384.74 & 386.97 & 387.48 \\
\hline- & \multicolumn{5}{|c|}{$\%$ Error } \\
\hline 0.00 & - & 0.00 & 0.00 & 0.00 & 0.00 \\
\hline 3.00 & - & -2.44 & -2.21 & -1.99 & -1.94 \\
\hline 6.00 & - & -13.69 & -13.34 & -12.98 & -12.90 \\
\hline 9.00 & - & -20.40 & -20.00 & -19.59 & -19.50 \\
\hline 12.00 & - & -20.15 & -19.71 & -19.26 & -19.16 \\
\hline 15.00 & - & -17.86 & -17.38 & -16.90 & -16.79 \\
\hline \multirow{7}{|c|}{ Temperature $\left({ }^{\circ} \mathbf{C}\right)$} \\
\cline { 2 - 6 } & $\mathbf{1 5 0 / 7 2}$ & $\mathbf{2 0 / 2 0}$ & $\mathbf{2 5 / 1 2}$ & $\mathbf{5 0 / 2 5}$ & $\mathbf{1 0 0 / 5 0}$ \\
\hline 0.00 & 25.00 & 25.00 & 25.00 & 25.00 & 25.00 \\
\hline 3.00 & 42.96 & 46.60 & 46.62 & 46.43 & 46.09 \\
\hline 6.00 & 88.00 & 101.11 & 101.16 & 100.52 & 99.28 \\
\hline 9.00 & 155.96 & 185.29 & 185.41 & 183.93 & 181.10 \\
\hline 12.00 & 254.90 & 310.35 & 310.57 & 307.83 & 302.57 \\
\hline 15.00 & 382.03 & 477.60 & 477.98 & 473.48 & 464.88 \\
\hline- & \multicolumn{5}{|c|}{$\%$ Error } \\
\hline 0.00 & 0.00 & 0.00 & 0.00 & 0.00 & 0.00 \\
\hline 3.00 & -2.49 & 5.78 & 5.82 & 5.39 & 4.62 \\
\hline 6.00 & -13.77 & -0.92 & -0.88 & -1.50 & -2.72 \\
\hline 9.00 & -20.49 & -5.53 & -5.47 & -6.23 & -7.67 \\
\hline 12.00 & -20.25 & -2.90 & -2.83 & -3.69 & -5.33 \\
\hline 15.00 & -17.96 & 2.56 & 2.64 & 1.67 & -0.17 \\
\hline
\end{tabular}


Table 18:

Large hotplate - results

\begin{tabular}{|c|c|c|c|c|c|}
\hline \multirow{2}{*}{$\begin{array}{c}\text { Voltage } \\
(\text { V) }\end{array}$} & \multicolumn{5}{|c|}{ Temperature $\left({ }^{\circ} \mathbf{C}\right)$} \\
\cline { 2 - 6 } & Expt. & $\mathbf{1 0 0 / 4 8}$ & $\mathbf{1 2 5} / \mathbf{6 0}$ & $\mathbf{1 5 0 / 7 2}$ & $\mathbf{1 2 5 / 9 0}$ \\
\hline 0.00 & 25.00 & 25.00 & 25.00 & 25.00 & 25.00 \\
\hline 3.00 & 40.04 & 43.58 & 43.34 & 43.09 & 43.13 \\
\hline 6.00 & 85.45 & 90.21 & 89.29 & 88.40 & 88.56 \\
\hline 9.00 & 158.61 & 160.19 & 158.19 & 156.29 & 156.64 \\
\hline 12.00 & 255.77 & 262.41 & 258.79 & 255.28 & 255.93 \\
\hline 15.00 & 371.44 & 393.82 & 388.16 & 382.68 & 383.69 \\
\hline- & \multicolumn{5}{|c|}{$\%$ Error } \\
\hline 0.00 & - & 0.00 & 0.00 & 0.00 & 0.00 \\
\hline 3.00 & - & 8.84 & 8.24 & 7.62 & 7.72 \\
\hline 6.00 & - & 5.57 & 4.49 & 3.45 & 3.64 \\
\hline 9.00 & - & 1.00 & -0.26 & -1.46 & -1.24 \\
\hline 12.00 & - & 2.60 & 1.18 & -0.19 & 0.06 \\
\hline 15.00 & - & 6.03 & 4.50 & 3.03 & 3.30 \\
\hline \multirow{7}{|c|}{ Temperature $\left({ }^{\circ} \mathbf{C}\right)$} \\
\cline { 2 - 6 } & $\mathbf{1 2 5 / 3 0}$ & $\mathbf{4 7 / 2 3}$ & $\mathbf{1 2 0} / 60$ & $\mathbf{2 0 0 / 1 0 0}$ & $\mathbf{2 5 0 / 1 2 5}$ \\
\hline 0.00 & 25.00 & 25.00 & 25.00 & 25.00 & 25.00 \\
\hline 3.00 & 43.54 & 45.52 & 44.64 & 43.84 & 43.36 \\
\hline 6.00 & 90.04 & 97.48 & 94.24 & 91.25 & 89.49 \\
\hline 9.00 & 159.81 & 175.90 & 168.74 & 162.46 & 158.88 \\
\hline 12.00 & 261.72 & 290.22 & 277.45 & 265.87 & 258.90 \\
\hline 15.00 & 392.74 & 437.13 & 417.18 & 399.12 & 388.22 \\
\hline- & \multicolumn{5}{|c|}{$\%$ Error } \\
\hline 0.00 & 0.00 & 0.00 & 0.00 & 0.00 & 0.00 \\
\hline 3.00 & 8.74 & 13.69 & 11.49 & 9.49 & 8.29 \\
\hline 6.00 & 5.37 & 14.08 & 10.29 & 6.82 & 4.73 \\
\hline 9.00 & 0.76 & 10.90 & 6.39 & 2.43 & 0.17 \\
\hline 12.00 & 2.33 & 13.47 & 8.48 & 3.95 & 1.22 \\
\hline 15.00 & 5.73 & 17.69 & 12.31 & 7.45 & 4.52 \\
\hline
\end{tabular}


Very large errors of up to $20 \%$ are noted for the medium hotplate (Table 17 ). For calculated $h$ however, the error drops to around $\pm 5 \%$. The values of $(25 / 12)$ and $(50 / 25)$ both provide quite good matches for the medium hotplate.

The large hotplate by far shows noticeable differences for different values of the convection coefficients. This underlines the fact that convection plays a major role in heat transfer at this size. The $h$ values of $(250 / 125)$ provide the best match with experimental results with a maximum error variation of $8 \%$. For the referenced values a similar range of $8 \%$ is provided by $(125 / 30)$. However the ratio of $4: 1$ for the top to bottom surfaces seems impractical as from the equations we know that the ratio of top to bottom surface is 2:1 (Eqs. 28 and 29).

The results show that the calculated values of $h$ provide a much better result than the nominal values. This is to be expected since the nominal values are referenced from other works and meant for a different hotplate.

One noticeable feature of the error curves is the consistency displayed by all the curves in their shape. The error is high at the lower voltages ( $3 \mathrm{~V})$ and then decreases towards $9 \mathrm{~V}$, after which it increases steadily with increasing input voltage. Some of the curves fall through the zero mark and display a negative value at around $9 \mathrm{~V}$. 


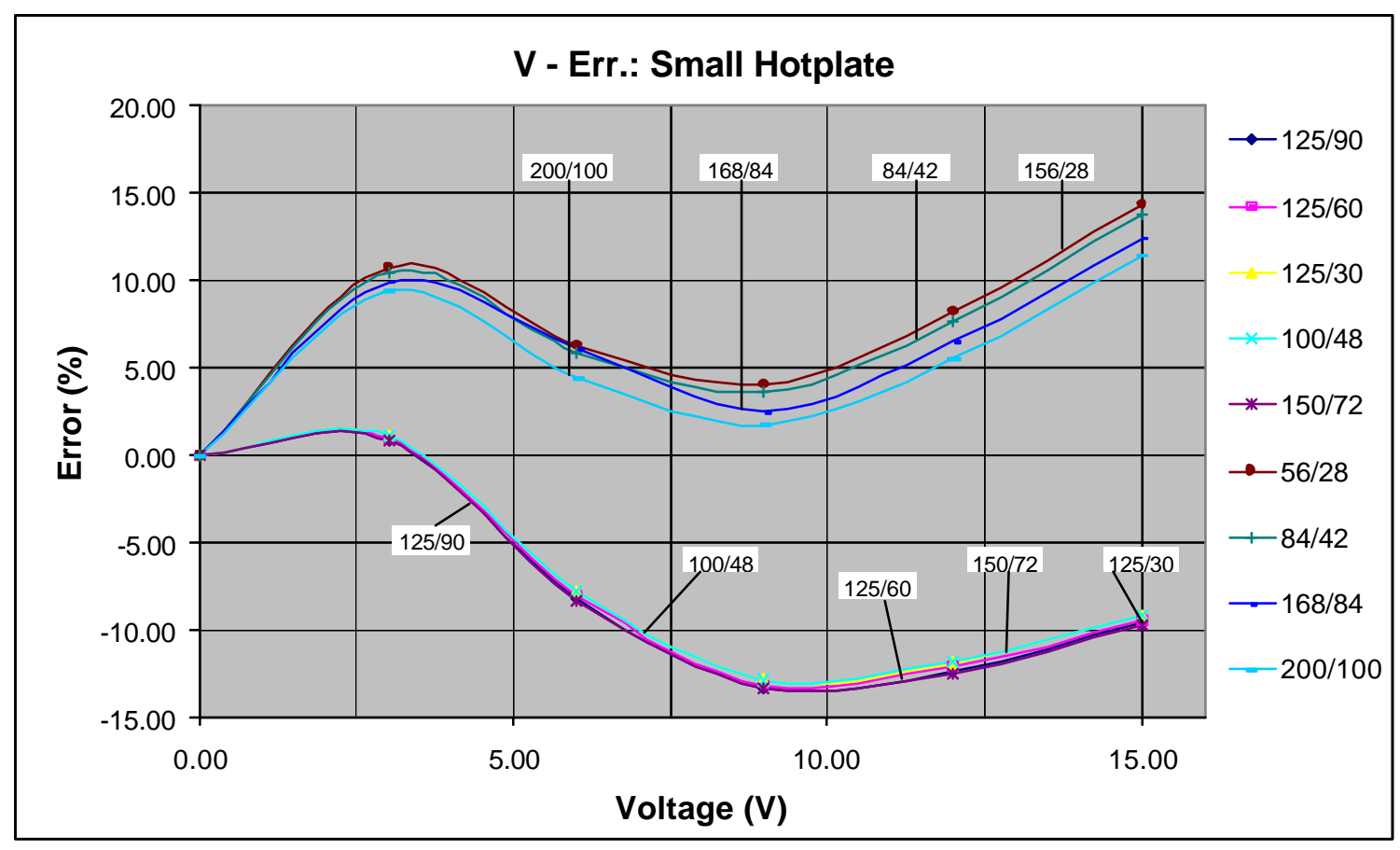

Figure 37: Error curves - small hotplate

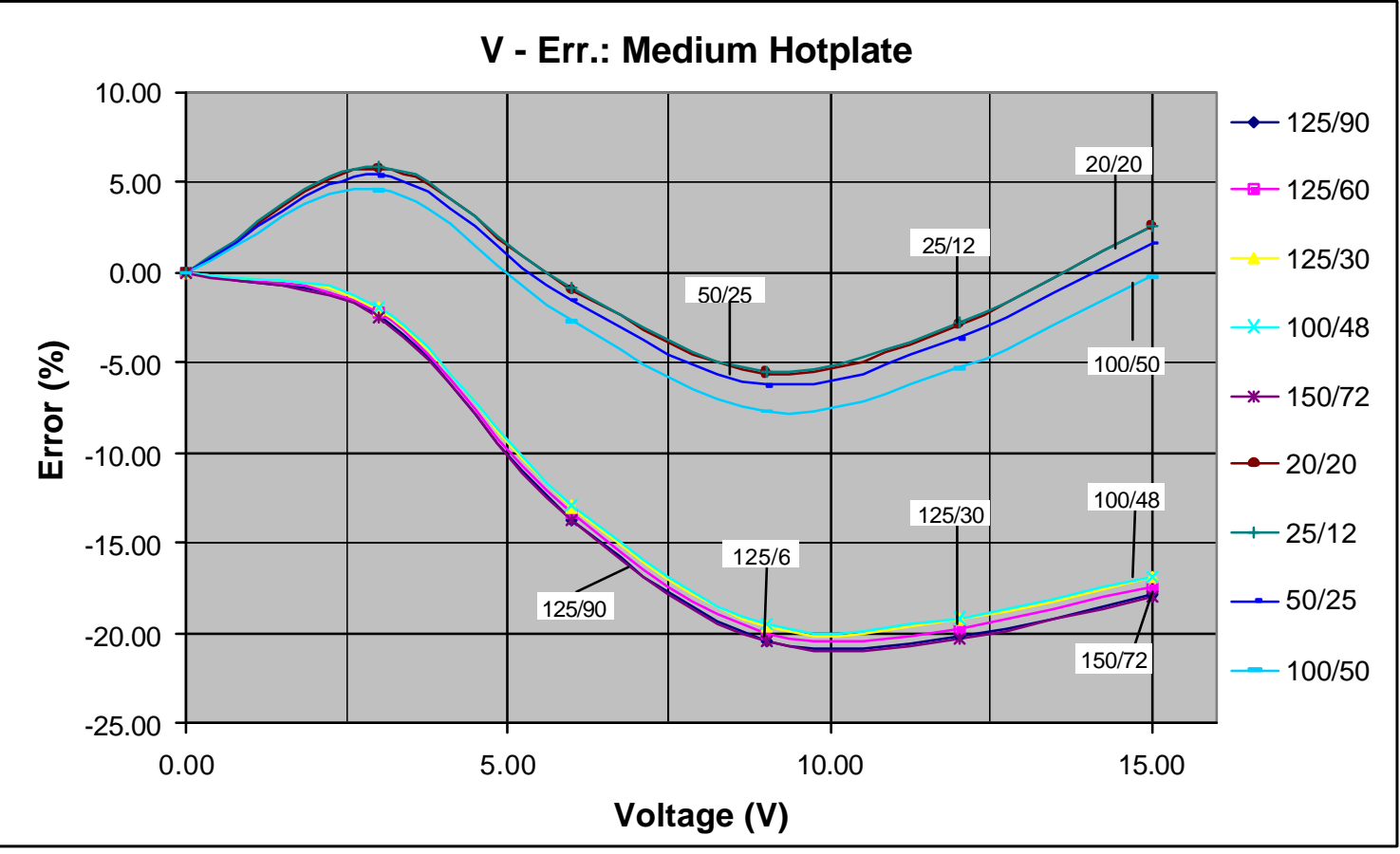

Figure 38: Error curves - medium hotplate 


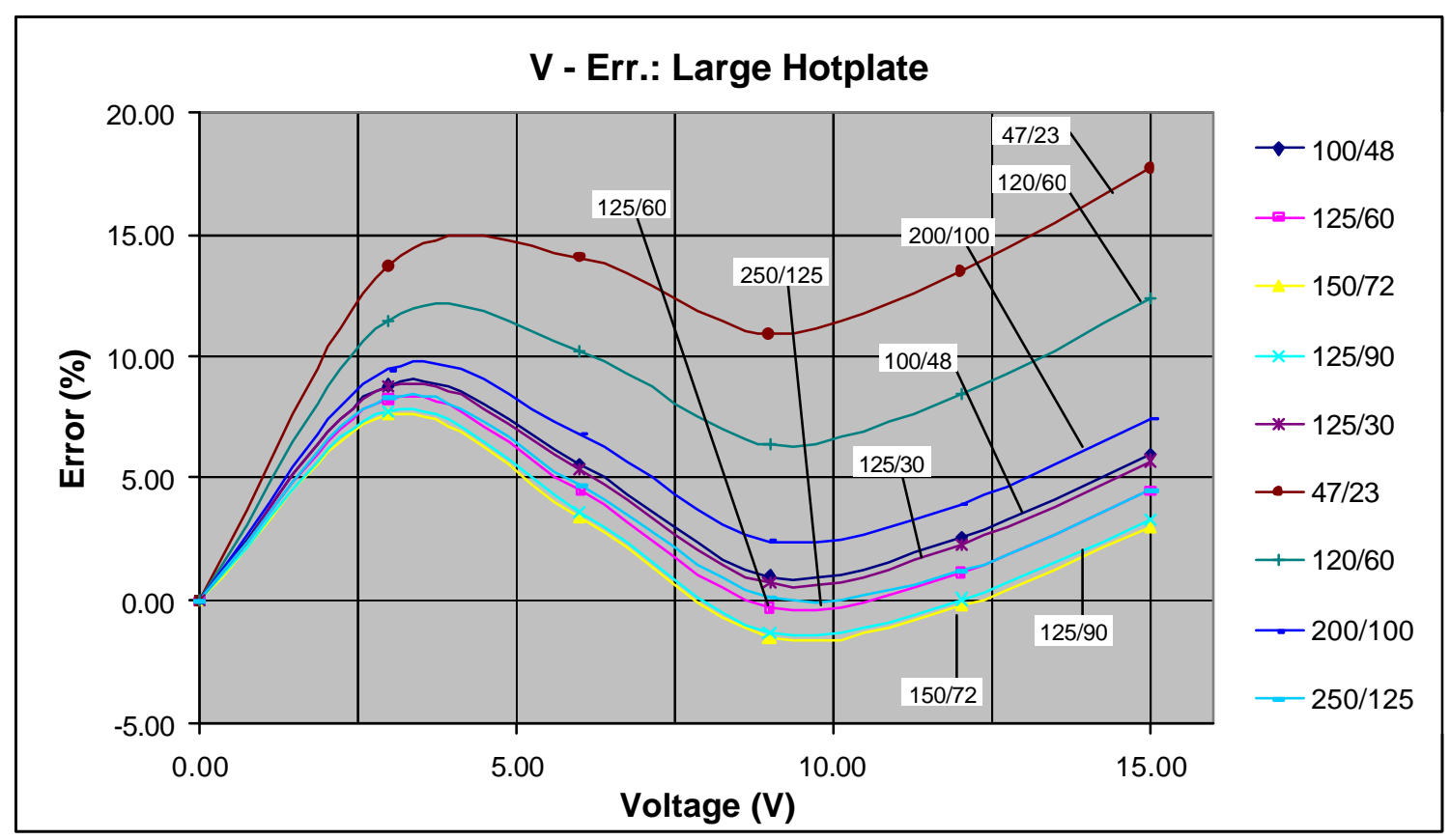

Figure 39: Error curves - large hotplate 


\section{CHAPTER 5 \\ CONCLUSIONS AND RECOMMENDATIONS}

As seen from the data, the convection coefficients affect the large hotplate more than the medium and small hotplates. This is clear from the error curves, which deviate for different values of ' $h$ '.

The 'error' is the difference between the simulated results and the experimental data. One conclusion that can be drawn from this is that convection plays a minor role in heat transfer as the size decreases. This supports previous work [9] where the convective loss falls from nearly $70 \%$ for a $1.8 \mathrm{~mm}^{2}$ hotplate to less than $35 \%$ for a $0.8 \mathrm{~mm}^{2}$ plate. At the small and medium sizes, in absence of convection, conduction would come into play. Also, since the size of the hotplates is at the micron scale, power loss due to radiation is negligible [11].

Due to the very small size of the heaters, testing the temperature of these devices requires highly sophisticated equipment like Infra Red (IR) cameras with high resolution lenses. An additional test resistor can be integrated into the die, which can be used as a thermometer. An alternative would be to build a sensing plate (an extra layer) into the device design [21, 22].

The procedure employed in this research to obtain temperature data is indirect, i.e. the temperature is deduced from two different data sets. As there are 
two - three steps to calculating the data, a small error at any step is magnified and would affect the final results.

The testing and characterization of these devices was done at atmospheric pressure. Testing can be done in vacuum [23], where the modes of heat transfer are conduction and radiation due to absence of fluid. If fluid flow is forced then data for forced convection can be obtained. Also, the devices in this research are tested horizontally, if they are tested vertically the results could be quite different as the fluid interaction increases. For a vertical plate, heat transfer due to free convection is higher than for a horizontal plate.

One possible reason for the errors is the question on material properties of the device layers. There is no measured data from the device itself regarding ts material properties. In future runs, test structures (eg. for thermal conductivity) can be fabricated on the chip to measure the data in-house [23, $24 \& 25]$.

One of the flaws noted was the uneven scaling between the different components of the three sizes (Table 1). To obtain better performance from these hotplates, the sizes can be increased to accommodate two - three on one die. Limitations to this would be the MOSIS fabrication technology, which uses only certain type of materials. The structure may not be capable of holding up when released due to the stress build within the suspended layers. MOSIS however, has the advantage of integrating electronics in the same chip as the MEMS devices. Since micro-scale heat transfer depends on the design type, geometry and ambient conditions, there is scope for future work and development for this design of hotplates. 


\section{REFERENCES}

[1] F. P. Incropera and D. P. DeWitt, Introduction to Heat Transfer, John Wiley \& Sons; pp2-10.

[2] W. M. Rohsenow, J. P. Hartnett and Y. I. Cho, Handbook of Heat Transfer, McGraw-Hill; pp1.1-1.10.

[3] S. Semancik, R. E. Cavicchi, M. C. Wheeler, J. E. Tiffany, G. E. Poirier, R. M. Walton, J. S. Suehle, B. Panchapakesan and D. L. DeVoe, "Microhotplate platforms for chemical sensor research". Sensors and Actuators B: Chemical, (Volume 77, Issues 1-2), 15 June 2001, pp579-591.

[4] M. Gaitan, M. Parameswran, R. B. Johnson and R. Chung, "Commercial CMOS Foundry Thermal Display for Dynamic Thermal Scene Simulation. SPIE Volume 1969, pp363-369.

[5] Cs. Dücsö, É. Vázsonyi, M. Ádám, I. Szabó, I. Bársony, J. G. E. Gardeniers and $A$. van den Berg, "Porous silicon bulk micromachining for thermally isolated membrane formation", Sensors and Actuators A: Physical, (Volume 60, Issues 13), May 1997, pp235-239.

[6] Courtesy, loana Vosculescu, George Washington University.

\section{[7] http://www.mosis.org}

[8] Huey-Daw Wu, MEMS Hot-Plates Annual Report, Naval Research Laboratory, July 01, 2002 - May 31, 2003.

[9] S. Astié, A. M. Gué, E. Scheid, L. Lescouzères and A. Cassagnes, "Optimization of an integrated $\mathrm{SnO}_{2}$ gas sensor using a FEM simulator", Sensors and Actuators A: Physical, (Volume 69, Issue 3), 15 September 1998, pp 205211.

[10] Z Vizvary, P. Furjes and I. Barsony, " Three-Dimensional Finite Element Model for Thermomechanical Analysis of Hotplates". 
[11] D. Briand, M. A. Gretillat, B. van der Schoot and N. F. de Rooji, " Thermal Management of Micro-Hotplates using MEMCAD as Simulation Tool", Technical Proceedings of the MSM 2000 International Conference on Modeling and simulation of Microsystems. March 27-29, 2000.

[12] D. Briand, A. Krauss, B. van der Schoot, U. Weimar, N. Barsan, W. Göpel and N. F. de Rooij, "Design and fabrication of high-temperature micro-hotplates for drop-coated gas sensors", Sensors and Actuators B: Chemical, (Volume 68, Issues 1-3), 25 August 2000, Pages 223-233.

[13] ARCHITECTTM Reference Guide and Tutorials, MEMS and Microsystems System Level Design, CoventorWare 2003.

[14] DESIGNER ${ }^{\mathrm{TM}}$ Reference Guide and Tutorials, MEMS and Microsystems Physical Design, CoventorWare 2003.

[15] ANALYZER ${ }^{\mathrm{TM}}$ Tutorials, MEMS and Microsystems, CoventorWare 2003.

[16] ANALYZER ${ }^{\mathrm{TM}}$ Reference Guide, MEMS and Mcrosystems, CoventorWare 2003.

[17] ANALYZERTM Supplemental Reference Guide and Tutorials, MEMS and Microsystems, CoventorWare 2003.

[18] R. C. Jaeger, Fundamentals of Microfabrication, $2^{\text {nd }}$ Ed. Prentice Hall, 2002.

[19] S. Uma, A. D. McConnell, M. Ashegi, K. Kurabayashi and K. E. Goodson, "Temperature-Dependent Thermal Conductivity of Undoped Polycrystalline Silicon Layers", Fourteenth Symposium on Thermophysical Properties, June 25 30, 2000, Boulder, Colorado, USA.

[20] http://efunda.com/materials/elements/element info.cfm?Element ID=AI (as on March 25, 2004)

[21] J. S. Suehle, R. E. Cavicchi, M. Gaitan and S. Semancik, "Tin Oxide Gas Sensor Fabricated Using CMOS Micro-Hotplates and In-Situ Processing", IEEE Electron Device Letters, (Volume 14, No.3), March 1993.

[22] R. E. Cavicchi, J. S. Suehle, K. G. Kreider, M. Gaitan and P Chaparala, "Fast Temperature Programmed Sensing for Micro-Hotplate Gas Sensor", IEEE Electron Device Letters, (Volume 16, No. 6), June 1995. 
[23] O. M. Paul, J. Korvink and H. Baltes, "Determination of the thermal conductivity of CMOS IC polysilicon", Sensors and Actuators A: Physical, (Volume 41, Issues 1-3), 1 April 1994, Pages 161-164.

[24] F. DiMeo, R. E. Cavichi, S, Semancik, J. S. Suehle, N. H. Tea, J. Small, J. T. Armstrong and J. T. Kelliher, "In situ conductivity characterization of oxide thin film growth phenomena on microhotplates", Journal of Vacuum Science Technology A 16(1), Jan. / Feb. 1998.

[25] C. H. Mastrangelo, Y. C. Tai and R. S. Muller, "Thermophysical properties of low-residual stress, Silicon-rich, LPCVD silicon nitride films", Sensors and Actuators A: Physical, (Volume 23, Issues 1-3), April 1990, pp856-860. 


\title{
CURRICULUM VITAE
}

\author{
Name HIREN VRAJLAL TRADA \\ Address Paul C. Lutz Hall, Rm. 428 \\ Department of Electrical Engineering \\ University of Louisville \\ Louisville, KY 40292 \\ Date of Birth \\ May $24^{\text {th }}, 1977 ;$ India \\ Education July 1992-May 1996 \\ Diploma of Engineering in Electronics and Communication \\ Maharashtra State Board of Technical Examinations, Mumbai, India \\ July 1996-Dec. 1999 \\ Bachelor of Engineering (B.E.) in Electronics and Telecommunication \\ University of Mumbai, Mumbai, India
}

Aug. 2000-May 2004

Master of Science (M.S.) in Electrical Engineering

University of Louisville, Louisville, KY

Professional Jan. 2001 - Aug. 2003

Experience Teaching Assistant, Department of Electrical Engineering

University of Louisville, Louisville, KY

Courses Taught $\quad$ ECE 334 - Electronics I Lab., ECE 513 - Electronics II Lab., ECE 535 Instrumentation Electronics Lab.

Awards

Aug. 2003, University Fellowship

Research Interests Microelectromechanical Systems (MEMS), sensors, actuators, MEMS design, device simulation, process simulation, Deep Reactive Ion Etching (DRIE), mask design and generation, wireless communications, applying MEMS to wireless technology. circuit design, VLSI design.

Technical Skills

CAD design and simulation using Coventorware (MEMCAD), L-Edit (design layout), ACES (process simulation), VLSI design (Magic), PCB layout, VHDL programming, various fabrication processes, web designing, computer troubleshooting (hardware \& software). 
